Pure and Applied Mathematics Quarterly

Volume 5, Number 1

(Special Issue: In honor of

Jean-Pierre Serre, Part 2 of 2)

$375-434,2009$

\title{
On the p-adic Beilinson Conjecture for Number Fields
}

\author{
A. Besser, P. Buckingham, R. de Jeu and X.-F. Roblot \\ Dedicated to Jean-Pierre Serre on the occasion of his eightieth birthday.
}

\begin{abstract}
We formulate a conjectural $p$-adic analogue of Borel's theorem relating regulators for higher $K$-groups of number fields to special values of the corresponding $\zeta$-functions, using syntomic regulators and $p$-adic $L$ functions. We also formulate a corresponding conjecture for Artin motives, and state a conjecture about the precise relation between the $p$-adic and classical situations. Parts of the conjectures are proved when the number field (or Artin motive) is Abelian over the rationals, and all conjectures are verified numerically in various other cases.
\end{abstract}

Keywords: Beilinson conjecture, Borel's theorem, number field, Artin motive, syntomic regulator, $p$-adic $L$-function

\section{INTRODUCTION}

The Beilinson conjectures about special values of $L$-functions [2] are a far reaching generalization of the class number formula for the Dedekind zeta function. For every smooth, projective algebraic variety $X$ over the rationals it predicts the leading term of the Taylor expansion of $L\left(H^{i}(X), s\right)$ at certain points, up to a rational multiple, in terms of arithmetic information associated with $X$, namely, its algebraic K-groups $K_{j}(X)$ [42]. More generally, these conjectures can also be formulated for motives.

Received July 25, 2007.

2000 Mathematics Subject Classification. Primary: 19F27; secondary: 11G55, 11R42, 11R70 
There have been several important steps taken towards verification of these conjectures in various cases, although, strictly speaking they have only been verified completely in the case where $X$ is the spectrum of a number field, where they follow from famous theorems of Borel [10, 11].

To motivate what follows, let us briefly recall the conjecture that interests us the most (for an introduction see $[23,45]$ ). One associates with $X$ two cohomology groups. The first one is the Deligne cohomology $H_{\mathcal{D}}^{i}\left(X_{/ \mathbb{R}}, \mathbb{R}(n)\right)$, which is an $\mathbb{R}$-vector space. The second is "integral" motivic cohomology $H_{M}^{i}\left(X_{/ \mathbb{Z}}, \mathbb{Q}(n)\right)$, which may be defined as a certain subspace of $K_{2 n-i}(X) \otimes_{\mathbb{Z}} \mathbb{Q}$. There is a regulator map defined by Beilinson,

$$
H_{M}^{i}\left(X_{/ \mathbb{Z}}, \mathbb{Q}(n)\right) \rightarrow H_{\mathcal{D}}^{i}\left(X_{/ \mathbb{R}}, \mathbb{R}(n)\right)
$$

If $2 n>i+1$ then $\operatorname{det} H_{\mathcal{D}}^{i}\left(X_{/ \mathbb{R}}, \mathbb{R}(n)\right)$ has a rational structure coming from the relations between $H_{\mathcal{D}}^{i}\left(X_{\mathbb{R}}, \mathbb{R}(n)\right)$ and the de Rham and singular cohomology groups of $X[45, \mathrm{p} .30]$.

The first part of the conjecture is that the map in (1.1) induces an isomorphism between the left-hand side tensored with $\mathbb{R}$ and the right-hand side, and consequently provides a second rational structure on $\operatorname{det} H_{\mathcal{D}}^{i}\left(X_{/ \mathbb{R}}, \mathbb{R}(n)\right)$. The second part of the conjecture states that, assuming a suitable functional equation for $L\left(H^{i-1}(X), s\right)$, these two rational structures differ from each other by the leading term in the Taylor expansion of $L\left(H^{i-1}(X), s\right)$ at $s=i-n$. Because of the expected functional equation one can reformulate the conjecture in terms of $L\left(H^{i-1}(X), n\right)$ (see [2, Corollary 3.6.2] or [31, 4.12]).

As mentioned before, this conjecture has only been verified in the case of number fields, due to difficulties in the computation of motivic cohomology. What has been verified in several other cases is a form of the conjecture in which one assumes the first part. For this one finds $\operatorname{dim}_{\mathbb{R}} H_{\mathcal{D}}^{i}\left(X_{/ \mathbb{R}}, \mathbb{R}(n)\right)$ elements of $H_{M}^{i}\left(X_{/ \mathbb{Z}}, \mathbb{Q}(n)\right)$, checks that their images under (1.1) are independent, hence should form a basis of $H_{M}^{i}\left(X_{/ \mathbb{Z}}, \mathbb{Q}(n)\right)$ according to the first part of the conjecture, and verifies the second part using these elements.

The idea that there should be a $p$-adic analogue of Beilinson's conjectures has been around since the late 80 's. Such a conjecture was formulated and proved by Gros in $[29,30]$ in the case of Artin motives associated with Dirichlet characters. In the weak sense mentioned before, it was proved for certain CM elliptic curves 
in [17] (where the relation with the syntomic regulator is proved in [4] and further elucidated in [7]), and for elliptic modular forms it follows from Kato's work (see [46]).

The book [41] contains a very general conjecture about the existence and properties of $p$-adic $L$-functions, from which one can derive a $p$-adic Beilinson conjecture. Rather than explain this in full detail we shall give a sketch of this conjecture similar to the sketch above of the Beilinson conjecture.

For the $p$-adic Beilinson conjectures one has to replace Deligne cohomology with syntomic cohomology $[30,40,3]$, the Beilinson regulator with the syntomic regulator, and $L$-functions with $p$-adic $L$-functions.

Syntomic cohomology $H_{\mathrm{syn}}^{i}(Y, n)$ is defined for a smooth scheme $Y$ of finite type over a complete discrete valuation ring of mixed characteristic $(0, p)$ with perfect residue field. For the $\mathbb{Q}$-variety $X$ we obtain, for all but finitely many primes, a map

$$
H_{M}^{i}\left(X_{/ \mathbb{Z}}, \mathbb{Q}(n)\right) \rightarrow H_{\mathrm{syn}}^{i}(Y, n)
$$

where $Y$ is a smooth model for $X$ over $\mathbb{Z}_{p}$. This cohomology group is a $\mathbb{Q}_{p}$-vector space.

The theory of $p$-adic $L$-functions starts with Kubota and Leopoldt's $p$-adic $\zeta$-function [35], $\zeta_{p}(s)$, which is defined by interpolating special values of complex valued Dirichlet $L$-functions. This principle has been extended to $\zeta$ - and $L$ functions in various situations, resulting in corresponding $p$-adic functions for totally real number fields [1, 15, 22], CM fields [32, 33] and modular forms [37]. (Given the occasion, let us note that the approach of Deligne and Ribet using modular forms was initiated by Serre [47].)

The $p$-adic Beilinson conjecture therefore has many similarities with its complex counterpart. However, there is a very important difference. In general, when $2 n>i+1$, there is no hope that (1.2) induces an isomorphism after tensoring the left-hand side with $\mathbb{Q}_{p}$. To see this, consider a number field $k$ with ring of algebraic integers $\mathcal{O}_{k}$. By Borel's theorem (see Theorem 2.2) we have, in accordance with Beilinson's conjectures,

$$
\operatorname{dim}_{\mathbb{Q}} H_{M}^{1}\left(\operatorname{Spec}(k)_{/ \mathbb{Z}}, \mathbb{Q}(n)\right)=\left\{\begin{aligned}
r_{2} \text { when } n \geq 2 & \text { is even } \\
r_{1}+r_{2} \text { when } n \geq 2 & \text { is odd }
\end{aligned}\right.
$$


with $r_{1}$ (resp. $2 r_{2}$ ) the number of real (resp. complex) embeddings of $k$. Thus, motivic cohomology "knows" about the number of real and complex embeddings of $k$, as does, by its definition, Deligne cohomology, which in this case becomes $H_{\mathcal{D}}^{1}\left(\operatorname{Spec}(k)_{/ \mathbb{R}}, \mathbb{R}(n)\right) \cong\left\{\left(x_{\sigma}\right)_{\sigma: k \rightarrow \mathbb{C}}\right.$ in $\mathbb{R}(n-1)^{r_{1}+2 r_{2}}$ such that $\left.\overline{x_{\sigma}}=x_{\bar{\sigma}}\right\}$. But syntomic cohomology, which depends only on the completion of $k$ at $p$, does not. In this case, we obtain $H_{\text {syn }}^{1}\left(\operatorname{Spec}\left(\mathcal{O}_{k} \otimes_{\mathbb{Z}} \mathbb{Z}_{p}\right), n\right) \cong \mathbb{Q}_{p}^{r_{1}+2 r_{2}}$.

The solution to this problem suggested in [41] is to make the $p$-adic $L$-function depend on a subspace of syntomic cohomology which is complementary to the image of the regulator. While this might seem artificial, there are other reasons for choosing this solution. In most cases one chooses a particular subspace and obtains a special case of the conjecture.

In the important special case of a totally real number field, or, more generally, an Artin motive over $\mathbb{Q}$ associated with a Galois representation where the conjugacy class of complex conjugation acts trivially (let us call these totally real Artin motives), no such subspace is required when $n \geq 2$ is odd (see Proposition 3.12). The same holds for Artin motives where this conjugacy class acts as multiplication by -1 and $n \geq 2$ is even (we may think of those as the "negative part" of CM Artin motives).

If $\chi$ denotes the character associated with either representation, then the Beilinson conjecture relates the regulator of $K_{2 n-1}$ with the Artin $L$-function of $\chi$ at $n$. For the $p$-adic $L$-functions one has to consider $\chi \otimes \omega_{p}^{1-n}$ with $\omega_{p}$ the Teichmüller character for the prime number $p$. Then the fixed field of the kernel of the representation underlying $\chi \otimes \omega_{p}^{1-n}$ is totally real, and it is perhaps no coincidence that in precisely this case the existence of a $p$-adic $L$-function that is not identicaly zero has been established, by $[1,15,22]$ for the case of fields and by [28] for Artin motives.

The goal of the present work is to describe in detail the conjectures for the cases of totally real fields, totally real Artin motives, as well as the "negative part" of CM Artin motives, and describe the (conjectural) relation between the classical and $p$-adic conjectures. We test everything numerically in several cases, and also deduce most of the conjectures for Abelian Artin motives from work by Coleman [16].

There have been several developments that allow us to carry out this numerical verification. In [19] de Jeu proved part of Zagier's conjecture concerning a 
more explicit description of the $K$-theory (tensored with $\mathbb{Q}$ ) of number fields (see Section 4). While this conjecture is not known to give the $K$-theory of such fields in all cases, it does in practice. Thus it provides a way of computing them, and Paul Buckingham wrote a computer implementation for this. In [5] Besser and de Jeu computed the syntomic regulator for (essentially) the part of the $K$-theory of a number field described by Zagier's conjecture and showed that it is given by applying the $p$-adic polylogarithm. Those $p$-adic polylogarithms were invented by Coleman [16] using his theory of $p$-adic integration but are not so easy to compute. In [6] Besser and de Jeu devised an algorithm for this computation. Taken together, these developments allow us to compute (1.1) and (1.2) for number fields. Finally, building on earlier work in [44], Roblot has dealt with the computational aspects of computing $p$-adic $L$-functions for Abelian characters over $\mathbb{Q}$ or a real quadratic field [43].

This paper is organized as follows. In Section 2 we recall Borel's theorem as well as various facts about $L$-functions and $p$-adic $L$-functions, and formulate a conjectural $p$-adic analogue of Borel's theorem. In Section 3 we introduce Artin motives with coefficients in a number field $E$ in terms of representations of the Galois group, determine when the $\mathbb{Q}$-dimension of the left-hand side of (1.2) equals the $\mathbb{Q}_{p}$-dimension of the right-hand side (corresponding to equality in Proposition 3.12), define both classical and $p$-adic $L$-functions with coefficients in $E$, and formulate the motivic Beilinson conjecture with coefficients in $E$, Conjecture 3.18, a small part of which is the same as a conjecture by Gross. In Section 4 we describe the set-up for finding elements in the $K$-groups of number fields using Zagier's conjecture, and the classical and $p$-adic regulators on them. We also prove most of Conjecture 3.18 for Abelian Artin motives over $\mathbb{Q}$ (Proposition 4.17 and Remark 4.18). In Section 5 we discuss a few computational aspects of implementing Zagier's conjecture and describe the Artin motives that we consider later for the numerical examples, and in the process prove Gross's conjecture for Artin motives obtained from $S_{3}$ and $D_{8}$-extensions of $\mathbb{Q}$. Then in Section 6 we sketch how the $p$-adic $L$-functions can be computed in certain cases, and make the required Brauer induction explicit for the Artin motives we want to consider. Finally, the last section is devoted to the results of the numerical calculations for examples.

Amnon Besser, Paul Buckingham and Rob de Jeu would like to thank the EC network Arithmetic Algebraic Geometry for travel support. Rob de Jeu 
would like to thank the Tata Institute of Fundamental Research for a productive stay during which this paper was worked on, and the Nuffield Foundation for a grant under the Undergraduate Research Bursary programme (NUF-URB03) that enabled Paul Buckingham to develop a computer program for finding the necessary elements in $K$-theory. Amnon Besser would like to thank the Institute for Advanced Study in Princeton for a stay during which part of the paper was worked on, and the Bell companies Fellowship and the James D. Wolfensohn fund for financial support while at the institute. Finally, the authors would like to thank Alfred Weiss for very useful explanations about $p$-adic $L$-functions, and, in particular, for bringing to their attention the paper [28].

\section{Notation}

Throughout the paper, for an Abelian group $A$ we let $A_{\mathbb{Q}}$ denote $A \otimes_{\mathbb{Z}} \mathbb{Q}$. If $B$ is a subgroup of $\mathbb{C}$ and $n$ an integer then we let $B(n)=(2 \pi i)^{n} B \subseteq \mathbb{C}$. We normalize the absolute value $|\cdot|_{p}$ on the field of $p$-adic numbers $\mathbb{Q}_{p}$ by $|p|_{p}=p^{-1}$, and use the same notation for its respective extensions to an algebraic closure $\overline{\mathbb{Q}}_{p}$ of $\mathbb{Q}_{p}$ and a completion $\mathbb{C}_{p}=\hat{\mathbb{Q}}_{p}$ of $\overline{\mathbb{Q}}_{p}$.

\section{The $p$-Adic Beilinson conjecture for totally Real fieldS}

Let $k$ be a number field with $r_{1}$ real embeddings, $2 r_{2}$ complex embeddings, ring of algebraic integers $\mathcal{O}_{k}$, and discriminant $D_{k}$. As is well-known, $\mathcal{O}_{k}^{*}$ is a finitely generated Abelian group of rank $r=r_{1}+r_{2}-1$, and its regulator $R$ satisfies $w \sqrt{\left|D_{k}\right|} \operatorname{Res}_{s=1} \zeta_{k}(s)=2^{r_{1}}(2 \pi)^{r_{2}}\left|\mathrm{Cl}\left(\mathcal{O}_{k}\right)\right| R$, with $\mathrm{Cl}\left(\mathcal{O}_{k}\right)$ the class group of $\mathcal{O}_{k}$, and $w$ the number of roots of unity in $k$. Because $K_{0}\left(\mathcal{O}_{k}\right) \cong \mathrm{Cl}\left(\mathcal{O}_{k}\right) \oplus \mathbb{Z}$ and $K_{1}\left(\mathcal{O}_{k}\right) \cong \mathcal{O}_{k}^{*}$, so $\left|\mathrm{Cl}\left(\mathcal{O}_{k}\right)\right|=\left|K_{0}\left(\mathcal{O}_{k}\right)_{\text {tor }}\right|$ and $w=\left|K_{1}\left(\mathcal{O}_{k}\right)_{\text {tor }}\right|$, this can be interpreted as a statement about the $K$-theory of $\mathcal{O}_{k}$, and it is from this point of view that it can be generalized to $\zeta_{k}(n)$ for $n \geq 2$. Namely, in [42], Quillen proved that $K_{m}\left(\mathcal{O}_{k}\right)$ is a finitely generated Abelian group for all $m$. Borel in [10] computed its rank when $m \geq 2$. For $m$ even this rank is zero, but for odd $m$ it is $r_{1}+r_{2}$ or $r_{2}$, and in [11] he showed that a suitably defined regulator of $K_{2 n-1}\left(\mathcal{O}_{k}\right)$ is related to $\zeta_{k}(n)$. Since $K_{2 n-1}\left(\mathcal{O}_{k}\right) \stackrel{\sim}{\rightarrow} K_{2 n-1}(k)$ when $n \geq 2$ we can rephrase his results for $K_{2 n-1}\left(\mathcal{O}_{k}\right)$ in terms of $K_{2 n-1}(k)$. Also, we replace Borel's regulator map $\operatorname{reg}_{B}: K_{2 n-1}(\mathbb{C}) \rightarrow \mathbb{R}(n-1)(n \geq 2)$ with Beilinson's regulator map $\operatorname{reg}_{\infty}($ see $[45, \S 4]$ ), which is half the Borel map regulator by $[12$, Theorem 10.9]. Because $k \otimes_{\mathbb{Q}} \mathbb{C} \cong \oplus_{\sigma: k \rightarrow \mathbb{C}} \mathbb{C}$, and $n>\operatorname{dim} \operatorname{Spec}(k \otimes \mathbb{C})$, we obtain 
by $[45$, p.9]

$$
\begin{aligned}
H_{\mathcal{D}}^{1}\left(\operatorname{Spec}(k \otimes \mathbb{C})_{/ \mathbb{R}}, \mathbb{R}(n)\right) & \cong H^{0}\left(\operatorname{Spec}(k \otimes \mathbb{C})_{/ \mathbb{R}}, \mathbb{R}(n-1)\right) \\
& \cong\left(\bigoplus_{\sigma: k \rightarrow \mathbb{C}} \mathbb{R}(n-1)\right)^{+},
\end{aligned}
$$

which consists of those $\left(x_{\sigma}\right)_{\sigma}$ with $x_{\bar{\sigma}}=\overline{x_{\sigma}}$. Finally, for any embedding $\sigma: k \rightarrow \mathbb{C}$ we let $\sigma_{*}: K_{2 n-1}(k) \rightarrow K_{2 n-1}(\mathbb{C})$ be the induced map.

Theorem 2.2. (Borel) Let $k$ be a number field of degree $d$, with $r_{1}$ real embeddings and $2 r_{2}$ complex embeddings, and let $n \geq 2$. Then the rank $m_{n}$ of $K_{2 n-1}(k)$ equals $r_{2}$ if $n$ is even and $r_{1}+r_{2}$ if $n$ is odd. Moreover, the map

$$
\begin{aligned}
K_{2 n-1}(k) & \rightarrow \bigoplus_{\sigma: k \rightarrow \mathbb{C}} \mathbb{R}(n-1) \\
\alpha & \mapsto\left(\operatorname{reg}_{\infty} \circ \sigma_{*}(\alpha)\right)_{\sigma}
\end{aligned}
$$

embeds $K_{2 n-1}(k) /$ torsion as a lattice in $\left(\oplus_{\sigma} \mathbb{R}(n-1)\right)^{+} \cong \mathbb{R}^{m_{n}}$, and the volume $V_{n}(k)$ of a fundamental domain of this lattice satisfies

$$
\zeta_{k}(n) \sqrt{\left|D_{k}\right|}=q \pi^{n\left(d-m_{n}\right)} V_{n}(k)
$$

for some $q$ in $\mathbb{Q}^{*}$.

Remark 2.5. In Theorem $2.2 m_{n}=0$ precisely when $k$ is totally real and $n \geq 2$ is odd. In this case the given relation holds (with $V_{n}(k)=1$ ) by the Siegel-Klingen theorem [39, Chapter VII, Corollary 9.9].

This theorem is equivalent with Beilinson's conjecture for $k$. In order to deal with this in detail (see Remarks 2.20 and 3.24) and in order to introduce $p$-adic $L$-functions we recall some facts about Artin $L$-functions [39, Chapter VII, $\S 10$ $12]$. Let $k$ be a number field, $d=[k: \mathbb{Q}]$, and let $\chi$ be a $\mathbb{C}$-valued Artin character of $\operatorname{Gal}(\bar{k} / k)$. For a prime number $\ell$ we define

$$
\operatorname{Eul}_{\ell}(s, \chi, k)=\prod_{\mathfrak{l} \mid \ell} \operatorname{Eul}_{\mathfrak{l}}(s, \chi, k)
$$

where $\operatorname{Eul}_{\mathfrak{l}}(s, \chi, k)$ is the reciprocal of the Euler factor for $\mathfrak{l}$ and the product is over primes $\mathfrak{l}$ of $k$ lying above $\ell$. Then for $s \in \mathbb{C}$ with $\operatorname{Re}(s)>1$ we can write the Artin $L$-function of $\chi$ as

$$
L(s, \chi, k)=\prod_{\ell} \operatorname{Eul}_{\ell}(s, \chi, k)^{-1} .
$$


For an infinite place $v$ of $k$ we let

$$
L_{v}(s, \chi, k)= \begin{cases}L_{\mathbb{C}}(s)^{\chi(1)} & \text { when } v \text { is complex, } \\ L_{\mathbb{R}}(s)^{n^{+}} L_{\mathbb{R}}(s+1)^{n^{-}} & \text {when } v \text { is real, }\end{cases}
$$

where $L_{\mathbb{R}}(s)=\pi^{-s / 2} \Gamma(s / 2), L_{\mathbb{C}}(s)=2(2 \pi)^{-s} \Gamma(s)$ and $n^{ \pm}=\frac{d}{2} \bar{\chi}(1) \pm \sum_{v} \frac{1}{2} \bar{\chi}\left(\operatorname{Fr}_{w}\right)$, with the sum taken over the real places $v$ of $k$ and $\mathrm{Fr}_{w}$ the generator of the image of $\operatorname{Gal}(\mathbb{C} / \mathbb{R})$ in $\operatorname{Gal}(\bar{k} / k)$ corresponding to any extension $w: \bar{k} \rightarrow \mathbb{C}$ of $v$. Then

$$
\Lambda(s, \chi, k)=L(s, \chi, k) \prod_{v \mid \infty} L_{v}(s, \chi, k)
$$

extends to a meromorphic function that satisfies the functional equation (cf. [39, p. 541])

$$
\Lambda(s, \chi, k)=W(\chi) C(\chi, k)^{1 / 2} C(\chi, k)^{-s} \Lambda(1-s, \bar{\chi}, k)
$$

with $W(\chi)$ a constant of absolute value 1 and $C(\chi, k)=\left|D_{k}\right|^{\chi(1)} \mathrm{Nm}_{k / \mathbb{Q}}(\mathfrak{f}(\chi, k))$ for $\mathfrak{f}(\chi, k)$ the Artin conductor of $\chi$. Therefore

$$
\begin{aligned}
L(1-s, \bar{\chi}, k)= & W(\bar{\chi}) C(\bar{\chi}, k)^{s-\frac{1}{2}}\left(2(2 \pi)^{-s} \Gamma(s)\right)^{d \bar{\chi}(1)} \\
& \times(\cos (\pi s / 2))^{n^{+}}(\sin (\pi s / 2))^{n^{-}} L(s, \chi, k) .
\end{aligned}
$$

Following [28, pp. 80-81] we shall now describe a $p$-adic $L$-function $L_{p}(s, \chi, k)$ when $k$ is a totally real number field, $p$ a prime and $\chi: \operatorname{Gal}(\bar{k} / k) \rightarrow \overline{\mathbb{Q}}_{p}$ a suitable Artin character. We begin with the case of 1-dimensional Artin characters.

If $\sigma: \overline{\mathbb{Q}}_{p} \rightarrow \mathbb{C}$ is any isomorphism then $\sigma \circ \chi$ is a complex Artin character, so we have the Artin $L$-function $L(s, \sigma \circ \chi, k)$. We may also view $\chi$ as a character of a suitable ray class group, so by [39, Corollary 9.9 and page 509] all $L(m, \sigma \circ \chi, k)$ for $m \in \mathbb{Z}_{\leq 0}$ are in $\mathbb{Q}(\sigma \circ \chi)$ and the values

$$
L^{*}(m, \chi, k)=\sigma^{-1}(L(m, \sigma \circ \chi, k))
$$

are independent of the choice of $\sigma$.

In the same way, we define

$$
\operatorname{Eul}_{\ell}^{*}(m, \chi, k)=\sigma^{-1}\left(\operatorname{Eul}_{\ell}(m, \sigma \circ \chi, k)\right),
$$

which is clearly independent of the choice of $\sigma$. To construct the $p$-adic $L$-function one finds a $p$-adic analytic or meromorphic function on an open ball around 0 that interpolates the values $L^{*}(m, \chi, k)$. For 1-dimensional $\chi$ with the fixed field $k_{\chi}$ of its kernel totally real this was achieved independently by Deligne and Ribet [22], Barsky [1], and Cassou-Noguès [14, 15]. We shall sketch a proof of the following 
theorem and Remark 2.13 below in Section 6. (When $k_{\chi}$ is not totally real the $p$-adic $L$-function is identically zero since the values interpolated are all zero by the functional equation of the $L$-function.)

Theorem 2.9. For $p$ prime, let $\mathcal{B}$ in $\mathbb{C}_{p}$ be the open ball with centre 0 and radius $q p^{-1 /(p-1)}$ where $q=p$ if $p>2$ and $q=4$ if $p=2$. If $k$ is a totally real number field and $\chi: \operatorname{Gal}(\bar{k} / k) \rightarrow \overline{\mathbb{Q}}_{p}$ a 1-dimensional Artin character, then there exists a unique $\mathbb{C}_{p}$-valued function $L_{p}(s, \chi, k)$ on $\mathcal{B}$ satisfying the following properties:

(1) $L_{p}(s, \chi, k)$ is analytic if $\chi$ is non-trivial and meromorphic with at most a simple pole at $s=1$ if $\chi$ is trival;

(2) if $m$ is a negative integer such that $m \equiv 1$ modulo $\varphi(q)$ then

$$
L_{p}(m, \chi, k)=\operatorname{Eul}_{p}^{*}(m, \chi, k) L^{*}(m, \chi, k) .
$$

If $\chi: \operatorname{Gal}(\bar{k} / k) \rightarrow \overline{\mathbb{Q}}_{p}$ is any Artin character then by Brauer's induction theorem $[39,(10.3)]$ there exist 1-dimensional Artin characters $\chi_{1}, \ldots, \chi_{t}$ on subgroups $G_{1}, \ldots, G_{t}$ of $\operatorname{Gal}(\bar{k} / k)$ of finite index, and integers $a_{1}, \ldots, a_{t}$, such that

$$
\chi=\sum_{i=1}^{t} a_{i} \operatorname{Ind}_{G_{i}}^{\operatorname{Gal}(\bar{k} / k)}\left(\chi_{i}\right) .
$$

If $k_{\chi}$ is totally real then we can assume that the same holds for the fixed fields $k_{i}$ of the $G_{i}$, and we define the $p$-adic $L$-function of $\chi$ by

$$
L_{p}(s, \chi, k)=\prod_{i=1}^{t} L_{p}\left(s, \chi_{i}, k_{i}\right)^{a_{i}},
$$

which is a meromorphic function on $\mathcal{B}$ (see Section 6). If $m$ is a negative integer satisfying $m \equiv 1$ modulo $\varphi(q)$ then the value $L_{p}(m, \chi, k)$ is defined and equals $\operatorname{Eul}_{p}^{*}(m, \chi, k) L^{*}(m, \chi, k)$ by well-known properties of Artin $L$-functions (see [39, Prop. 10.4(iv)]), showing the function is independent of how we express $\chi$ as a sum of induced 1-dimensional characters.

Remark 2.12. In [28] Greenberg proves that the Main Conjecture of Iwasawa theory implies the $p$-adic Artin conjecture, that is, that the $p$-adic $L$-function of an Artin character $\chi$ is analytic on the open ball $\mathcal{B}$ if it does not contain the trivial character, and has at most a simple pole at $s=1$ otherwise. It therefore follows from the proof of the Main Conjecture for $p$ odd by Wiles [53] that $L_{p}(n, \chi, k)$ 
for $p \neq 2$ is defined for all integers $n \neq 1$. In particular, the values of the $p$ adic $L$-functions in Conjecture 2.17 below exist by Theorem 2.9, and those in Conjecture 3.18 exist when $p \neq 2$ but have to be assumed to exist when $p=2$.

Remark 2.13. Let $W_{p} \subset \mathbb{Z}_{p}^{*}$ be the subgroup of $(p-1)$-th roots of unity if $p$ is odd and let $W_{2}=\{ \pm 1\}$. The Teichmüller character on $\operatorname{Gal}(\bar{k} / k)$ is defined as the composition

$$
\omega_{p}: \operatorname{Gal}(\bar{k} / k) \rightarrow \operatorname{Gal}(\overline{\mathbb{Q}} / \mathbb{Q}) \rightarrow \operatorname{Gal}\left(\mathbb{Q}\left(\mu_{q}\right) / \mathbb{Q}\right) \stackrel{\sim}{\rightarrow}(\mathbb{Z} / q \mathbb{Z})^{*} \stackrel{\sim}{\rightarrow} W_{p},
$$

where the last map sends an element of $(\mathbb{Z} / q \mathbb{Z})^{*}$ to the unique element of $W_{p}$ to which it is congruent modulo $q$. For an Artin character $\chi: \operatorname{Gal}(\bar{k} / k) \rightarrow \overline{\mathbb{Q}}_{p}$ and an integer $l, \chi \omega_{p}^{l}$ is also an Artin character. If $m \leq 0$ satisfies $l+m \equiv 1$ modulo $\varphi(q)$ and either $\chi$ is 1-dimensional or $k_{\chi}$ is totally real then $L_{p}\left(m, \chi \omega_{p}^{l}, k\right)=$ $\operatorname{Eul}_{p}^{*}(m, \chi, k) L^{*}(m, \chi, k)$.

The $p$-adic Beilinson conjecture is going to predict the special values of $p$-adic $L$-functions in terms of a $p$-adic regulator. The required regulator is the syntomic regulator [3, Theorem 7.5]. Let $F$ be a complete discretely valued field of characteristic 0 with perfect residue field of characteristic $p$ and let $X$ be a scheme that is smooth and of finite type over the valuation $\operatorname{ring} \mathcal{O}_{F}$. Then the above mentioned paper associates to $X$ its rigid syntomic cohomologies $H_{\mathrm{syn}}^{i}(X, n)$, as well as syntomic regulators (i.e., Chern characters) $\operatorname{reg}_{p}: K_{2 n-i}(X) \rightarrow H_{\mathrm{syn}}^{i}(X, n)$. In this work, unlike in [3], we shall need to change the base field $F$. We therefore prefer to denote the syntomic cohomology by $H_{\mathrm{syn}}^{i}\left(X / \mathcal{O}_{F}, n\right)$. For the formulation of the conjecture the following basic fact is required.

Lemma 2.15. We have $H_{\mathrm{syn}}^{1}\left(\operatorname{Spec}\left(\mathcal{O}_{F}\right) / \mathcal{O}_{F}, n\right) \cong F$, for all $n>0$ and consequently we have a syntomic regulator $\operatorname{reg}_{p}: K_{2 n-1}\left(\mathcal{O}_{F}\right) \rightarrow F$. Furthermore, the map $\operatorname{reg}_{p}$ commutes in the obvious way with finite extensions of fields and with automorphisms of such fields, provided that their residue fields are algebraic over the prime field.

Proof. The first claim follows from part 3 of [3, Proposition 8.6]. For the second claim we note that by part 4 of the same proposition we have in this case an isomorphism between syntomic and modified syntomic cohomology (the latter only exists under the additional assumption on the residue field). The compatibility with finite base changes now follows from the same result for modified syntomic 
cohomology in [3, Proposition 8.8]. The same holds for automorphisms, although not explicitly stated in the above reference, since the relevant base change results, e.g., Proposition 8.6.4, hold for this type of base change as well.

As a consequence of the lemma we can also, by abuse of notation, define for any complete discretely valued subfield $F \subset \overline{\mathbb{Q}}_{p}$ the regulator $\operatorname{reg}_{p}: K_{2 n-1}\left(\mathcal{O}_{F}\right) \rightarrow F$. In [5], two of the authors of the present work showed how one can sometimes compute the map $\operatorname{reg}_{p}$ by using $p$-adic polylogarithms.

We now restrict our attention to a totally real number field $k$ of degree $d$. Our goal will be to formulate a conjecture that is the $p$-adic analogue of Theorem 2.2 for $K_{2 n-1}(k)$ with $n \geq 2$. Since this $K$-group is torsion when $n$ is even but has rank $d$ when $n$ is odd, we only consider odd $n \geq 2$.

In preparation for the more general construction that will follow in Section 3 let us first reformulate Theorem 2.2 in this special case. Let $a_{1}, \ldots, a_{d}$ form a $\mathbb{Z}$-basis of $\mathcal{O}_{k}$ and let $\sigma_{1}^{\infty}, \ldots, \sigma_{d}^{\infty}$ be the embeddings of $k$ into $\mathbb{C}$. Then we define $D_{k}^{1 / 2, \infty}=$ $\operatorname{det}\left(\sigma_{i}^{\infty}\left(a_{j}\right)\right)$, a real root of the discriminant of $k$. Similarly, if $\alpha_{1}, \ldots, \alpha_{d}$ form a $\mathbb{Z}$-basis of $K_{2 n-1}(k) /$ torsion then we let $R_{n, \infty}(k)=\operatorname{det}\left(\operatorname{reg}_{\infty} \circ \sigma_{i *}^{\infty}\left(\alpha_{j}\right)\right)$. Then $D_{k}^{1 / 2, \infty}$ and $R_{n, \infty}(k)$ are well-defined up to sign, and the relation in Theorem 2.2 in this case is equivalent with

$$
\zeta_{k}(n) D_{k}^{1 / 2, \infty}=q(n, k) R_{n, \infty}(k)
$$

with $q(n, k)$ in $\mathbb{Q}^{*}$.

Now let $F \subset \overline{\mathbb{Q}}_{p}$ be the topological closure of the Galois closure of $k$ embedded in $\overline{\mathbb{Q}}_{p}$ in any way. If $\sigma_{1}^{p}, \ldots, \sigma_{d}^{p}$ are the embeddings of $k$ into $F$ then $D_{k}^{1 / 2, p}=$ $\operatorname{det}\left(\sigma_{i}^{p}\left(a_{j}\right)\right)$ is a root in $F$ of the discriminant of $k$. For $\sigma: k \rightarrow F$ an embedding we denote the induced map $K_{2 n-1}(k) \cong K_{2 n-1}\left(\mathcal{O}_{k}\right) \rightarrow K_{2 n-1}\left(\mathcal{O}_{F}\right)$ by $\sigma_{*}$. Then we define a $p$-adic regulator in $F$ by $R_{n, p}(k)=\operatorname{det}\left(\operatorname{reg}_{p} \circ \sigma_{i *}^{p}\left(\alpha_{j}\right)\right)$. Both $D_{k}^{1 / 2, p}$ and $R_{n, p}(k)$ are well-defined up to sign.

Remark 2.13 suggests that the role of $\zeta_{k}(n)$ in a $p$-adic analogue of Theorem 2.2 should be played by $L_{p}\left(n, \omega_{p}^{1-n}, k\right) / \operatorname{Eul}_{p}(n, k)$ where $\zeta_{k}(s)=\prod_{l} \operatorname{Eul}_{l}(s, k)^{-1}$ for $\operatorname{Re}(s)>1$, so we can hope that

$$
L_{p}\left(n, \omega_{p}^{1-n}, k\right) D_{k}^{1 / 2, p}=q_{p}(n, k) \operatorname{Eul}_{p}(n, k) R_{n, p}(k)
$$

for some $q_{p}(n, k)$ in $\mathbb{Q}^{*}$. 
More precisely, because $R_{n, \infty}(k) / D_{k}^{1 / 2, \infty}$ and $R_{n, p}(k) / D_{k}^{1 / 2, p}$ are invariant under reordering the $\sigma_{i}^{\infty}$ or $\sigma_{i}^{p}$, and transform in the same way if we change the bases of $\mathcal{O}_{k}$ and $K_{2 n-1}(k) /$ torsion, we can make the following conjecture.

Conjecture 2.17. For $k$ a totally real number field, $p$ prime, and $n \geq 2$ odd, we have, with notation as above:

(1) in $F$ the equality

$$
L_{p}\left(n, \omega_{p}^{1-n}, k\right) D_{k}^{1 / 2, p}=q_{p}(n, k) \operatorname{Eul}_{p}(n, k) R_{n, p}(k)
$$

holds for some $q_{p}(n, k)$ in $\mathbb{Q}^{*}$;

(2) in fact, $q_{p}(n, k)=q(n, k)$;

(3) $L_{p}\left(n, \omega_{p}^{1-n}, k\right)$ and $R_{n, p}(k)$ are non-zero.

As mentioned in the introduction, this, and the corresponding parts of Conjecture 3.18 below, can be deduced (with some effort) from a much more general conjecture of Perrin-Riou [41, 4.2.2].

Remark 2.18. The conjecture is similar to the result that the residue of $\zeta_{p}(s, k)$ at $s=1$ is related to the Leopoldt regulator of $\mathcal{O}_{k}^{*}$ through exactly the same formula as for the residue of $\zeta(s, k)$ and the Dirichlet regulator [18], with part(4) corresponding to the Leopoldt conjecture. However, we have not tried to determine if Colmez's normalization of the sign for the regulator is the same as here, especially given the sign error in the proof of Lemma 4.3 in loc. cit. (see Section 6).

Remark 2.19. In the definition of $R_{n, \infty}(k)$ and $R_{n, p}(k)$ we can use a basis of a subgroup of finite index of $K_{2 n-1}(k) /$ torsion or even a $\mathbb{Q}$-basis of $K_{2 n-1}(k)_{\mathbb{Q}}$, without affecting the rationality of $q(n, k), q_{p}(n, k)$ or their equality. Similarly we can replace the $\mathbb{Z}$-basis of $\mathcal{O}_{k}$ with a $\mathbb{Q}$-basis of $k$ in the definitions of $D_{k}^{1 / 2, \infty}$ and $D_{k}^{1 / 2, p}$.

Remark 2.20. As is well-known, for $k$ and $n$ as in Conjecture 2.17, by (2.6) $\zeta_{k}(s)$ at $s=1-n$ has a zero of order $d$ and the first non-zero coefficient in its Taylor expansion, $\zeta_{k}^{\sharp}(1-n)$, equals $(2 \pi i)^{d(1-n)}((n-1) ! / 2)^{d} D_{k}^{n-1 / 2} \zeta_{k}(n)$. If we take $D_{k}^{1 / 2, \infty}=D_{k}^{1 / 2}$ in (2.16) then we obtain

$$
\zeta_{k}^{\sharp}(1-n)=((n-1) ! / 2)^{d} q(n, k) D_{k}^{n-1} \widetilde{R}_{n, \infty}(k)
$$


for Beilinson's renormalized regulator $\widetilde{R}_{n, \infty}(k)=(2 \pi i)^{d(1-n)} R_{n, \infty}(k)$. In computer calculations $((n-1) ! / 2)^{d} q(n, k) D_{k}^{n-1}$ often has only relatively small prime factors, so the larger prime factors in $q(n, k)$ correspond to $D_{k}^{1-n}$. This phenomenon also occurs in the calculations for Conjecture 3.18 below (see Remark 7.8).

Remark 2.21. (1) The thought that $L_{p}\left(n, \omega_{p}^{1-n}, k\right)$ is non-zero for $n \geq 2$ and odd when $k$ is a totally real Abelian extension of $\mathbb{Q}$ is mentioned by C. Soulé in $[49,3.4]$.

(2) F. Calegari [13] (see also [8]) proved that, for $p=2$ and $3, \zeta_{p}(3)$, which in those cases equals $L_{p}\left(3, \omega_{p}^{-2}, \mathbb{Q}\right)$, is irrational. (More results along these lines are described in Remark 3.20(3) below.)

(3) Parts (1) and (2) of Conjecture 2.17 hold when $k$ is a totally real Abelian number field, and in fact the corresponding parts of a much stronger conjecture that we shall describe in Section 3 hold for cyclotomic fields (see Proposition 4.17).

(4) We numerically verified part (3) of Conjecture 2.17, and its more refined version Conjecture 3.18(4) below, in certain cases; see Remark 4.19 and Section 7.

\section{A motivic Version of the CONJeCture}

If $E$ is any extension of $\mathbb{Q}$, and $k / \mathbb{Q}$ is finite and Galois with Galois group $G$, then we let $M^{E}=E \otimes_{\mathbb{Q}} k$ and $K_{2 n-1}(k)_{E}=E \otimes_{\mathbb{Q}} K_{2 n-1}(k)_{\mathbb{Q}}$, which are $E[G]$ modules. The goal of this section is to refine Conjecture 2.17 to a conjecture for Artin motives with coefficients in $E$, or equivalently, idempotents in the group ring $E[G]$, when $E$ is a number field.

Definition 3.1. If $\pi$ is an idempotent in $E[G]$ then we write $M_{\pi}^{E}$ for $\pi M^{E}$ and $K_{2 n-1}\left(M_{\pi}^{E}\right)$ for $\pi K_{2 n-1}(k)_{E}$.

Now fix an embedding $\phi_{\infty}: k \rightarrow \mathbb{C}$. The pairing $G \times k \rightarrow \mathbb{C}$ mapping $(\sigma, a)$ to $\phi_{\infty}(\sigma(a))$ leads to an $E$-bilinear pairing

$$
\begin{aligned}
E[G] \times M^{E} & \rightarrow E \otimes \mathbb{Q} \mathbb{C} \\
\left(e \sigma, e^{\prime} \otimes a\right) & \mapsto e e^{\prime} \otimes \phi_{\infty}(\sigma(a)) .
\end{aligned}
$$

and we consider its restriction

$$
(\cdot, \cdot)_{\infty}: E[G] \pi \times M_{\pi}^{E} \rightarrow E \otimes_{\mathbb{Q}} \mathbb{C} .
$$


Similarly, replacing $\phi_{\infty}$ with a fixed embedding $\phi_{p}: k \rightarrow \overline{\mathbb{Q}}_{p}$ we obtain

$$
(\cdot, \cdot)_{p}: E[G] \pi \times M_{\pi}^{E} \rightarrow E \otimes_{\mathbb{Q}} F .
$$

where $F \subset \overline{\mathbb{Q}}_{p}$ is the topological closure of $\phi_{p}(k)$, which is independent of $\phi_{p}$ since $k / \mathbb{Q}$ is Galois.

Lemma 3.3. Let $\pi$ in $E[G]$ be an idempotent. Then $\operatorname{dim}_{E}\left(M_{\pi}^{E}\right)=\operatorname{dim}_{E}(E[G] \pi)$.

Proof. Since $\pi^{2}=\pi,(3.2)$ is identically 0 on $E[G] \pi \times M_{1-\pi}^{E}$ and $E[G](1-\pi) \times M_{\pi}^{E}$. But the determinant of this pairing is, up to multiplication by $E^{*}$, equal to $D_{k}^{1 / 2, \infty}$, hence non-zero. This implies the lemma.

We now introduce pairings similar to $(\cdot, \cdot)_{\infty}$ and $(\cdot, \cdot)_{p}$ but replacing $M_{\pi}^{E}$ with $K_{2 n-1}\left(M_{\pi}^{E}\right)$. If we denote the map $K_{2 n-1}(k)_{\mathbb{Q}} \rightarrow K_{2 n-1}(\mathbb{C})_{\mathbb{Q}}$ induced by $\phi_{\infty}$ by $\phi_{\infty *}$ and let $\Phi_{\infty}$ be the composition with the Beilinson regulator map reg ${ }_{\infty}$ for $\mathbb{C}$

$$
\Phi_{\infty}=\operatorname{reg}_{\infty} \circ \phi_{\infty *}: K_{2 n-1}(k)_{\mathbb{Q}} \rightarrow K_{2 n-1}(\mathbb{C})_{\mathbb{Q}} \rightarrow \mathbb{R}(n-1) \subset \mathbb{C},
$$

then the pairing $G \times K_{2 n-1}(k)_{\mathbb{Q}} \rightarrow \mathbb{C}$ given by mapping $(\sigma, \alpha)$ to $\Phi_{\infty}(\sigma(\alpha))$ gives rise to an $E$-bilinear pairing

$$
\begin{aligned}
E[G] \times K_{2 n-1}\left(M^{E}\right) & \rightarrow E \otimes \mathbb{Q} \mathbb{C} \\
\left(e \sigma, e^{\prime} \otimes \alpha\right) & \mapsto e e^{\prime} \otimes \Phi_{\infty}(\sigma(\alpha))
\end{aligned}
$$

and we consider its restriction

$$
[\cdot, \cdot]_{\infty}: E[G] \pi \times K_{2 n-1}\left(M_{\pi}^{E}\right) \rightarrow E \otimes_{\mathbb{Q}} \mathbb{C} .
$$

By Lemma 2.15 and the definition of $F$ the syntomic regulator gives us

$$
\operatorname{reg}_{p}: K_{2 n-1}\left(\mathcal{O}_{F}\right)_{\mathbb{Q}} \rightarrow F .
$$

If we write $\phi_{p *}$ for the composition $K_{2 n-1}(k)_{\mathbb{Q}} \cong K_{2 n-1}\left(\mathcal{O}_{k}\right)_{\mathbb{Q}} \rightarrow K_{2 n-1}\left(\mathcal{O}_{F}\right)_{\mathbb{Q}}$, with the second map induced by $\phi_{p}: k \rightarrow F$, and let $\Phi_{p}$ be the composition

$$
\operatorname{reg}_{p} \circ \phi_{p *}: K_{2 n-1}(k)_{\mathbb{Q}} \rightarrow K_{2 n-1}(F)_{\mathbb{Q}} \rightarrow F,
$$

then we can similarly obtain a pairing

$$
[\cdot, \cdot]_{p}: E[G] \pi \times K_{2 n-1}\left(M_{\pi}^{E}\right) \rightarrow E \otimes_{\mathbb{Q}} F
$$

We now fix ordered $E$-bases of $E[G] \pi, M_{\pi}^{E}$, and $K_{2 n-1}\left(M_{\pi}^{E}\right)$. 
Definition 3.5. For $*=\infty$ or $p$ we let $D\left(M_{\pi}^{E}\right)^{1 / 2, *}$ be the determinant of the pairing $(\cdot, \cdot)_{*}$, computed with respect to our fixed bases of $E[G] \pi$ and $M_{\pi}^{E}$.

Note that $D\left(M_{\pi}^{E}\right)^{1 / 2, \infty}$ is non-zero by the proof of Lemma 3.3 , and the same argument works for $D\left(M_{\pi}^{E}\right)^{1 / 2, p}$.

Definition 3.6. If $\operatorname{dim}_{E}(E[G] \pi)$ equals $\operatorname{dim}_{E}\left(K_{2 n-1}\left(M_{\pi}^{E}\right)\right)$ then for $*=\infty$ or $p$ we let $R_{n, *}\left(M_{\pi}^{E}\right)$ be the determinant of the pairing $[\cdot, \cdot]_{*}$, computed with respect to our fixed bases of $E[G] \pi$ and $K_{2 n-1}\left(M_{\pi}^{E}\right)$.

For future use we prove the following.

Lemma 3.7. If $\operatorname{dim}_{E}(E[G] \pi)=\operatorname{dim}_{E}\left(K_{2 n-1}\left(M_{\pi}^{E}\right)\right)$ then

(1) $R_{n, \infty}\left(M_{\pi}^{E}\right) / D\left(M_{\pi}^{E}\right)^{1 / 2, \infty}$ is independent of the basis of $E[G] \pi$, of $\phi_{\infty}$, and lies in $E \otimes \mathbb{R}$;

(2) $R_{n, p}\left(M_{\pi}^{E}\right) / D\left(M_{\pi}^{E}\right)^{1 / 2, p}$ is independent of the basis of $E[G] \pi$, of $\phi_{p}$, and lies in $E \otimes \mathbb{Q}_{p}$.

Proof. We prove the second statement, the proof of the first being entirely similar. Choosing a different $E$-basis of $E[G] \pi$ corresponds to letting an $E$-linear transformation act on $E[G] \pi$, and in the given quotient the resulting determinant cancels. Since $k / \mathbb{Q}$ is Galois we get all possible embeddings of $k$ into $\overline{\mathbb{Q}}_{p}$ by replacing $\phi_{p}$ with $\phi_{p} \circ \sigma$ for $\sigma$ in $G$. For both $R_{n, p}\left(M_{\pi}^{E}\right)$ and $D\left(M_{\pi}^{E}\right)^{1 / 2, p}$ this corresponds to letting $\sigma$ act on $E[G] \pi$, and the resulting determinant cancels as before. That the quotient lies in $E \otimes \mathbb{Q}_{p}$ follows because it lies in $E \otimes F$ and we have just proved that it is invariant under $\operatorname{Gal}\left(F / \mathbb{Q}_{p}\right)$ by Lemma 2.15 .

We now investigate when the two dimensions in Definition 3.6 are equal. The answer is given by Proposition 3.12 below, but we need some preliminary results.

Proposition 3.8. If $k / \mathbb{Q}$ is a finite Galois extension with Galois group $G$ and $\pi$ is an idempotent in $E[G]$ then $\operatorname{dim}_{E}\left(K_{2 n-1}\left(M_{\pi}^{E}\right)\right) \leq \operatorname{dim}_{E}(E[G] \pi)$. Equality holds precisely when any (hence every) $\tau$ in the image in $G$ of the conjugacy class of complex conjugation in $\operatorname{Gal}(\overline{\mathbb{Q}} / \mathbb{Q})$ acts by $(-1)^{n-1}$ on $E[G] \pi$.

Proof. For the statement we can first replace $E$ by a finitely generated subfield since $\pi$ contains only finitely many elements of $E$, next embed $E$ into $\mathbb{C}$ and use this embedding to enlarge $E$ to $\mathbb{C}$. So we may assume that $E=\mathbb{C}$. 
According to Theorem 2.2 the pairing (3.4) gives an injection

$$
\begin{aligned}
\mathbb{R} \otimes_{\mathbb{Q}} K_{2 n-1}(k)_{\mathbb{Q}} & \rightarrow \mathbb{R}[G]^{\vee}=\operatorname{Hom}_{\mathbb{R}}(\mathbb{R}[G], \mathbb{R}) \\
\alpha & \mapsto f_{\alpha} \text { with } f_{\alpha}(\sigma)=\frac{1}{(2 \pi i)^{n-1}} \Phi_{\infty}(\sigma(\alpha))
\end{aligned}
$$

and by extending the coefficients we get an injection $\mathbb{C} \otimes_{\mathbb{Q}} K_{2 n-1}(k)_{\mathbb{Q}} \rightarrow \mathbb{C}[G]^{\vee}$. The image of $\pi\left(K_{2 n-1}(k)_{\mathbb{Q}} \otimes_{\mathbb{Q}} \mathbb{C}\right)$ under the last map vanishes on $\mathbb{C}[G](1-\pi)$ since $(1-\pi) \pi=0$ so that $\pi K_{2 n-1}(k)_{\mathbb{Q}} \otimes_{\mathbb{Q}} \mathbb{C}$ injects into $(\mathbb{C}[G] / \mathbb{C}[G](1-\pi))^{\vee} \cong$ $(\mathbb{C}[G] \pi)^{\vee}$, which proves our first inequality.

As for equality, we know by Theorem 2.2 that (3.9) has as its image the subspace of $\mathbb{R}[G]^{\vee}$ where, under the action of $\operatorname{Gal}(\overline{\mathbb{Q}} / \mathbb{Q})$ on $\mathbb{R}[G]^{\vee}$ via $(\tilde{\sigma} f)(\sigma)=$ $f\left(\tilde{\sigma}^{-1} \sigma\right)$, the conjucagy class of complex conjugation in $\operatorname{Gal}(\overline{\mathbb{Q}} / \mathbb{Q})$ acts as multiplication by $(-1)^{n-1}$. The same will therefore hold with complex coefficients. Since any element $\tau$ in the conjugacy class of complex conjugation has order 1 or $2, \mathbb{C}[G] \pi$ decomposes into eigenspaces for the eigenvalues \pm 1 and the desired equality can only hold if $\tau$ acts as multiplication by $(-1)^{n-1}$ on all of $\mathbb{C}[G] \pi$.

We now determine precisely when the equality of dimensions as in Proposition 3.8 can occur, and for this we need a preliminary result.

Proposition 3.10. Let $\psi$ be a representation of $\operatorname{Gal}(\overline{\mathbb{Q}} / \mathbb{Q})$ over $\overline{\mathbb{Q}}$ that factorizes through the Galois group of a finite Galois extension of $\mathbb{Q}$ and for which $\psi(\tau)$ acts as multiplication by $(-1)^{n-1}$ for any $\tau$ in the conjugacy class of complex conjugation in $\operatorname{Gal}(\overline{\mathbb{Q}} / \mathbb{Q})$. Then the fixed field of $\operatorname{Ker}(\psi)$ is a finite Galois extension of $\mathbb{Q}$ that is totally real if $n$ is odd, and $C M$ if $n$ is even.

Proof. That the fixed field $k$ of $\operatorname{Ker}(\psi)$ is a finite Galois extension of $\mathbb{Q}$ is clear. When $n$ is odd $\psi(\tau)=1$ for any $\tau$ in the conjugacy class of complex conjugation so that $k$ is totally real. For even $n$ we let $\omega$ be the composition $\operatorname{Gal}(\overline{\mathbb{Q}} / \mathbb{Q}) \rightarrow$ $\operatorname{Gal}\left(\mathbb{Q}\left(\mu_{4}\right) / \mathbb{Q}\right) \stackrel{\sim}{\rightarrow}\{ \pm 1\} \subset \overline{\mathbb{Q}}^{*}$. Then $\operatorname{Ker}(\psi \omega)$ contains $\tau$ and $\operatorname{Ker}(\psi) \cap \operatorname{Ker}(\omega)$, hence its fixed field is a totally real Galois extension $k^{+}$of $\mathbb{Q}$, contained in $k\left(\mu_{4}\right)$. Similarly $k$ is contained in the CM field $k^{+}\left(\mu_{4}\right)$ so, since $k$ is not totally real, it must be CM.

Remark 3.11. For a CM field $k$ with $k / \mathbb{Q}$ Galois, its maximal totally real subfield is Galois over $\mathbb{Q}$ and is the fixed field of an element of order two in the centre of $\operatorname{Gal}(k / \mathbb{Q})$, which we shall refer to as the complex conjugation of $k$. 
Proposition 3.12. Let $k / \mathbb{Q}$ be a finite Galois extension with Galois group $G$, $E$ any extension of $\mathbb{Q}$, and $\pi$ an idempotent of $E[G]$. Let $k^{\prime}$ be the fixed field of the kernel of the representation of $G$ on $E[G] \pi$. Then for $n \geq 2$ the equality $\operatorname{dim}_{E}(E[G] \pi)=\operatorname{dim}_{E}\left(K_{2 n-1}\left(M_{\pi}^{E}\right)\right)$ holds precisely in the following cases:

(1) $k^{\prime}$ is totally real and $n$ is odd;

(2) $k^{\prime}$ is a CM field, $n$ is even, and the complex conjugation of $k^{\prime}$ acts on $E[G] \pi$ as multiplication by -1 .

Proof. From Propositions 3.8 and 3.10 we see that there cannot be any other cases. Conversely, by Proposition 3.8 equality holds in both.

We now recall and introduce some terminology for later use.

Definition 3.13. Let $G$ be a finite group and $E$ any extension of $\mathbb{Q}$.

(1) If $\pi$ is a central idempotent of $E[G]$ such that $E[G] \pi$ is a minimal (nonzero) 2-sided ideal of $E[G]$ then $\pi$ is a primitive central idempotent.

(2) If $\pi$ is a primitive central idempotent of $E[G]$ such that $E[G] \pi \cong M_{m}(E)$ as $E$-algebras for some $m$, then we call a primitive idempotent corresponding to $\pi$ any element in $E[G] \pi \subseteq E[G]$ that maps to a matrix in $M_{m}(E)$ that is conjugate to a matrix with 1 in the upper left corner and 0 's elsewhere. (Since all automorphisms of $M_{m}(E)$ as $E$-algebra are inner this is independent of the isomorphism $E[G] \pi \cong M_{m}(E)$.)

Remark 3.14. (1) If $E[G] \pi \cong M_{m}(E)$ then any idempotent in $E[G] \pi$ can be written as a sum of orthogonal primitive idempotents corresponding to $\pi$ as one sees immediately by diagonalizing the matrix $A$ that $\pi$ maps to, which satisfies $A^{2}=A$.

(2) If $G=\operatorname{Gal}(k / \mathbb{Q})$ and $\pi$ is a primitive central idempotent of $E[G]$ then the dimensions of $E[G] \tilde{\pi}$ and $\tilde{\pi} K_{2 n-1}(k)_{E}$ are equal for some non-zero idempotent $\tilde{\pi}$ in $E[G] \pi$ if and only if the same holds for one (hence any) primitive idempotent corresponding to $\pi$. Indeed, the dimensions for $\tilde{\pi}$ do not change if we replace it with a conjugate in $E[G]$, and they add up for sums of orthogonal idempotents.

We now introduce $L$-functions, both classical and $p$-adic, in the following context. Let $E$ be a finite extension of $\mathbb{Q}, k$ a number field, and consider a character $\psi$ of a representation of $G=\operatorname{Gal}(\bar{k} / k)$ on a finite dimensional $E$-vector space $V$ 
that factorizes through $\operatorname{Gal}\left(\bar{k} / k^{\prime}\right)$ for some finite extension $k^{\prime}$ of $k$. Then for every embedding $\sigma: k \rightarrow \mathbb{C}$ we have the Artin $L$-function $L(s, \sigma(\psi), k)$, with for every prime $\mathfrak{P}$ of $k$ the reciprocal $\operatorname{Eul}_{\mathfrak{P}}(s, \sigma(\psi), k)$ of the Euler factor corresponding to $\mathfrak{P}$. Under the natural isomorphism $E \otimes_{\mathbb{Q}} \mathbb{C} \stackrel{\sim}{\rightarrow} \oplus_{\sigma} \mathbb{C}_{\sigma}$ the $L(s, \sigma(\psi), k)$ correspond to a canonical $E \otimes_{\mathbb{Q}} \mathbb{C}$-valued $L$-function that we denote by $L(s, \psi \otimes \mathrm{id}, k)$. Similarly, for every prime $\mathfrak{P}$ of $k$ we have an $E \otimes_{\mathbb{Q}} \mathbb{C}$-valued $\operatorname{Eul}_{\mathfrak{P}}(s, \psi \otimes \mathrm{id}, k)$ corresponding to the $\operatorname{Eul}_{\mathfrak{P}}(s, \sigma(\psi), k)$.

We now move on to the $p$-adic $L$-functions, and assume that $k$ is totally real, $p$ a prime number, $a$ any integer and $\omega_{p}$ the Teichmüller character $\operatorname{Gal}(\overline{\mathbb{Q}} / k) \rightarrow \mathbb{Q}_{p}^{*}$ (see (2.14)). If $\tau: E \rightarrow \overline{\mathbb{Q}}_{p}$ is an embedding and $k_{\tau(\psi) \omega_{p}^{a}}$ is totally real then from Section 2 we have the $p$-adic $L$-function $L_{p}\left(s, \tau(\psi) \omega_{p}^{a}, k\right)$, which is not identically zero. In this case, using the natural isomorphism $E \otimes_{\mathbb{Q}} \overline{\mathbb{Q}}_{p} \stackrel{\sim}{\rightarrow} \oplus_{\tau} \overline{\mathbb{Q}}_{p, \tau}$, they give us an $E \otimes_{\mathbb{Q}} \overline{\mathbb{Q}}_{p}$-valued $p$-adic $L$-function on $\mathbb{Z}_{p}$ or $\mathbb{Z}_{p} \backslash\{1\}$ that we denote by $L_{p}\left(s, \psi \otimes \omega_{p}^{a}, k\right)$.

Lemma 3.15. The values of $L_{p}\left(s, \psi \otimes \omega_{p}^{a}, k\right)$ are in $E \otimes_{\mathbb{Q}} \mathbb{Q}_{p}$.

Proof. Using Brauer induction for $\psi$ (cf. (2.10)) it suffices to prove this when $\psi$ is 1-dimensional. But then for each $\tau: E \rightarrow \overline{\mathbb{Q}}_{p}$ the function $L_{p}\left(s, \tau(\psi) \omega_{p}^{a}, k\right)$ can be described as in (6.3) with $l=-1$, from which the result is clear.

Remark 3.16. It follows from Remark 2.13 that $L_{p}\left(s, \psi \otimes \omega_{p}^{a}, k\right)$ satisfies

$$
L_{p}\left(m, \psi \otimes \omega_{p}^{a}, k\right)=\operatorname{Eul}_{p}(m, \psi \otimes \mathrm{id}, k) L(m, \psi \otimes \mathrm{id}, k)
$$

for integers $m \leq 0$ congruent to $1-a$ modulo $\phi(q)$, where both sides lie in $E=E \otimes_{\mathbb{Q}} \mathbb{Q}$ inside $E \otimes_{\mathbb{Q}} \mathbb{Q}_{p}$ and $E \otimes_{\mathbb{Q}} \mathbb{C}$ respectively, and

$$
\operatorname{Eul}_{p}(s, \psi \otimes \mathrm{id}, k)=\prod_{\mathfrak{P} \mid p} \operatorname{Eul}_{\mathfrak{P}}(s, \psi \otimes \mathrm{id}, k),
$$

the product being over all primes of $k$ dividing $p$.

Remark 3.17. If $E=\mathbb{Q}$ then we shall identify $E \otimes_{\mathbb{Q}} \mathbb{C}$ with $\mathbb{C}$, and write $L(s, \psi, k)$, etc., instead of $L(s, \psi \otimes$ id, $k)$, etc. Similarly we identify $E \otimes_{\mathbb{Q}} \mathbb{Q}_{p}$ with $\mathbb{Q}_{p}$ and write $L_{p}\left(s, \psi \omega_{p}^{a}, k\right)$ instead of $L_{p}\left(s, \psi \otimes \omega_{p}^{a}, k\right)$.

We now have all the ingredients for the generalization and refinement of Conjecture 2.17. Starting with a finite Galois extension $k / \mathbb{Q}$ with Galois group $G$, 
$E$ a finite extension of $\mathbb{Q}$, and $\pi$ an idempotent in $E[G]$, we let $\psi_{\pi}$ be the natural representation of $\operatorname{Gal}(\overline{\mathbb{Q}} / \mathbb{Q})$ on $E[G] \pi$ and $\chi_{\pi}$ its associated character. If $\operatorname{dim}_{E}(E[G] \pi)=\operatorname{dim}_{E}\left(\pi K_{2 n-1}(k)_{E}\right)$ for some $n \geq 2$ then, for any prime $p$ and any embedding $\tau: E \rightarrow \overline{\mathbb{Q}}_{p}, \tau\left(\psi_{\pi}\right) \omega_{p}^{1-n}$ is trivial on the conjugacy class of complex conjugation in $\operatorname{Gal}(\overline{\mathbb{Q}} / \mathbb{Q})$ by Proposition 3.8. In particular, $\mathbb{Q}_{\tau\left(\psi_{\pi}\right) \omega_{p}^{1-n}}$ is totally real and therefore $L_{p}\left(s, \chi_{\pi} \otimes \omega_{p}^{1-n}, \mathbb{Q}\right)$ is not identically zero. With $F \subset \overline{\mathbb{Q}}_{p}$ the topological closure of $\phi_{p}(k)$ as before, using ordered bases for $E[G] \pi, M_{\pi}^{E}$ and $K_{2 n-1}\left(M_{\pi}^{E}\right)$, we have $D\left(M_{\pi}^{E}\right)^{1 / 2, \infty}$ and the regulator $R_{n, \infty}\left(M_{\pi}^{E}\right)$ in $E \otimes \mathbb{C}$ as well as $D\left(M_{\pi}^{E}\right)^{1 / 2, p}$ and $R_{n, p}\left(M_{\pi}^{E}\right)$ in $E \otimes F$.

Conjecture 3.18. Let notation be as above. If $n \geq 2$ and $\operatorname{dim}_{E}(E[G] \pi)$ is equal to $\operatorname{dim}_{E}\left(K_{2 n-1}\left(M_{\pi}^{E}\right)\right)$, then

(1) in $E \otimes_{\mathbb{Q}} \mathbb{C}$ we have

$$
L\left(n, \chi_{\pi} \otimes \mathrm{id}, \mathbb{Q}\right) D\left(M_{\pi}^{E}\right)^{1 / 2, \infty}=e\left(n, M_{\pi}^{E}\right) R_{n, \infty}\left(M_{\pi}^{E}\right)
$$

for some e $\left(n, M_{\pi}^{E}\right)$ in $\left(E \otimes_{\mathbb{Q}} \mathbb{Q}\right)^{*}$;

(2) in $E \otimes_{\mathbb{Q}} F$ we have

$$
\begin{aligned}
& L_{p}\left(n, \chi_{\pi} \otimes \omega_{p}^{1-n}, \mathbb{Q}\right) D\left(M_{\pi}^{E}\right)^{1 / 2, p}=e_{p}\left(n, M_{\pi}^{E}\right) \operatorname{Eul}_{p}\left(n, \chi_{\pi} \otimes \mathrm{id}, \mathbb{Q}\right) R_{n, p}\left(M_{\pi}^{E}\right) \\
& \text { for some } e_{p}\left(n, M_{\pi}^{E}\right) \text { in }\left(E \otimes_{\mathbb{Q}}\right)^{*} ; \\
& \text { (3) in fact, } e_{p}\left(n, M_{\pi}^{E}\right)=e\left(n, M_{\pi}^{E}\right) ; \\
& \text { (4) } L_{p}\left(n, \chi_{\pi} \otimes \omega_{p}^{1-n}, \mathbb{Q}\right) \text { and } R_{n, p}\left(M_{\pi}^{E}\right) \text { are units in } E \otimes_{\mathbb{Q}} \mathbb{Q}_{p} \text { and } E \otimes_{\mathbb{Q}} F \\
& \text { respectively. }
\end{aligned}
$$

Remark 3.19. (1) One sees as in the proof of Lemma 3.7 that the validity of each part of the conjecture is independent of the chosen bases of $E[G] \pi, M_{\pi}^{E}$ and $K_{2 n-1}\left(M_{\pi}^{E}\right)$.

(2) Since $E \otimes_{\mathbb{Q}} \mathbb{C} \stackrel{\sim}{\rightarrow} \oplus_{\sigma} E_{\sigma}$, where the sum is over all embeddings $\sigma: E \rightarrow \mathbb{C}$, an identity in $E \otimes_{\mathbb{Q}} \mathbb{C}$ is equivalent to the corresponding identities for all such embeddings. The same holds if we replace $\mathbb{C}$ with $\overline{\mathbb{Q}}_{p}$.

In Remark 3.24 we shall make explicit how part (1) of this conjecture is equivalent with Beilinson's conjecture [2, Conjecture 3.4] for an Artin motive associated with $\pi$. First, we make various remarks about its dependence on $E$, etc., and on its relation with Conjecture 2.17. 
Remark 3.20. (1) Conjecture 3.18(1) can be formulated for any idempotent $\pi$ if $k / \mathbb{Q}$ is any finite Galois extension (see the end of Section 5), in which case it is a conjecture by Gross (see [38, p. 210]). In that case it was proved by Beilinson if the action of $G=\operatorname{Gal}(k / \mathbb{Q})$ on $E[G] \pi$ is Abelian (see loc. cit.). One can also prove that it holds for any $\pi$ in $E[G]$ if this action factors through $S_{3}$ or $D_{8}$ (see Proposition 5.9).

(2) If the action of $G$ on $E[G]$ is Abelian then parts (1)-(3) of Conjecture 3.18 hold for any $\pi$ to which the conjecture applies (see Remark 4.18).

(3) Extending and simplifying earlier work by F. Calegari [13], F. Beukers in [8] proves that $\zeta_{p}(2)$ is irrational when $p=2$ or 3 . This value equals $L_{p}\left(2, \chi \omega_{p}^{-1}, \mathbb{Q}\right)$ with $\chi$ the primitive character on $(\mathbb{Z} / 4 \mathbb{Z})^{*}$ for $p=2$, and the primitive character on $(\mathbb{Z} / 3 \mathbb{Z})^{*}$ for $p=3$. Moreover, if $\chi$ is the odd primitive character on $(\mathbb{Z} / 8 \mathbb{Z})^{*}$ then he also shows that $L_{2}\left(2, \chi \omega_{2}^{-1}, \mathbb{Q}\right)$ is irrational. It follows that the conjecture holds in full for $\pi$ corresponding to the non-trivial representation of $\operatorname{Gal}(k / \mathbb{Q})$ and $n=2$ when $(k, p)$ is one of $(\mathbb{Q}(\sqrt{-1}), 2),(\mathbb{Q}(\sqrt{-2}), 2)$ and $(\mathbb{Q}(\sqrt{-3}), 3)$.

(4) We have verified numerically that part (4) of the conjecture holds in certain cases; see Remark 4.19 as well as Section 7.

Remark 3.21. (1) If $\pi=\pi_{1}+\cdots+\pi_{m}$ with $\pi_{i}^{2}=\pi_{i}$ and $\pi_{i} \pi_{j}=0$ when $i \neq j$, then the conjecture for $\pi$ is implied by the conjecture for all $\pi_{i}$ because $D\left(\oplus_{i} M_{\pi_{i}}^{E}\right)^{1 / 2, \infty}=\prod_{i} D\left(M_{\pi_{i}}^{E}\right)^{1 / 2, \infty}$, etc., as one easily sees by using bases.

(2) If $\pi$ is in $E[G]$ and $E^{\prime}$ is an extension of $E$, then we may view $\pi$ as an element of $E^{\prime}[G]$ as well, and the conjectures for $M_{i}^{E}$ and $M_{i}^{E^{\prime}}$ are equivalent: we can use the same bases over $E^{\prime}$ as over $E$, so that $D\left(M_{i}^{E}\right)^{1 / 2, \infty}=D\left(M_{i}^{E^{\prime}}\right)^{1 / 2, \infty}$ in $E \otimes \mathbb{C} \subseteq E^{\prime} \otimes \mathbb{C}$, and the same holds for all the other ingredients (including the $L$-functions).

(3) By comparing bases one sees immediately that if $\pi$ and $\pi^{\prime}$ are conjugate under the action of $E[G]^{*}$ then the conjectures for $\pi$ and for $\pi^{\prime}$ are equivalent.

(4) If, for a primitive central idempotent $\pi_{i}$ of $E[G], E[G] \pi_{i} \cong M_{m}(E)$ for some $m$, and $\pi$ is a primitive idempotent corresponding to $\pi_{i}$, then the conjecture for $\pi_{i}$ is implied by the conjecture for $\pi$. Indeed, we can decompose $\pi_{i}$ into a sum of orthogonal primitive idempotents as in Remark 3.14(1), and the truth of the conjecture for $\pi_{i}$ is implied by its truth for each such primitive idempotent. But all such primitive idempotents are conjugate to $\pi$ hence (3) above applies. 
Remark 3.22. If $k / \mathbb{Q}$ is a Galois extension with Galois group $G$ and $H$ a subgroup of $G$ with fixed field $k^{H}$, then $K_{m}(k)_{\mathbb{Q}}^{H}=K_{m}\left(k^{H}\right)_{\mathbb{Q}}$, a result known as Galois descent. With $\pi_{H}=|H|^{-1} \sum_{h \in H} h$, an idempotent in $E[G]$, this implies that $\pi_{H} K_{m}(k)=K_{m}\left(k^{H}\right)$.

Remark 3.23. (1) Let $k / \mathbb{Q}$ be a Galois extension with Galois group $G$. If $N$ is a normal subgroup of $G$ corresponding to $k^{\prime}=k^{N}$ and $\pi_{N}=|N|^{-1} \sum_{h \in N} h$, then $\pi_{N}^{2}=\pi_{N}$ and the natural map $\phi: E[G] \rightarrow E[G / N]$ has kernel $E[G]\left(1-\pi_{N}\right)$ and induces an isomorphism $E[G] \pi_{N} \rightarrow E[G / N]$. Indeed, it is clear that $\pi_{N}$ is central in $E[G]$ since $N$ is normal in $G$ and that $1-\pi_{N}$ is in the kernel of $\phi$. Also, since $N$ acts trivially on $E[G] \pi_{N}$ this is an $E[G / N]$-module generated by one element, so its dimension over $E$ cannot be bigger than $|G / N|$. Since $\phi$ is obviously surjective our claims follow. Therefore, in this situation, for any idempotent $\pi$ in $E[G / N]$ there is a canonical idempotent $\tilde{\pi}$ lifting $\pi$ to $E[G] \pi_{N}$, and the natural map $E[G] \tilde{\pi} \rightarrow E[G / N] \pi$ is an isomorphism of $E[G]$ - and $E[G / N]$-modules.

Then the statements of Conjecture 3.18 for $\pi$ in $E[G / N]$ or for $\tilde{\pi}$ in $E[G]$ are equivalent. Namely,

$$
\tilde{\pi} K_{2 n-1}(k)_{E}=\tilde{\pi} \pi_{N} K_{2 n-1}(k)_{E}=\tilde{\pi} K_{2 n-1}\left(k^{\prime}\right)_{E}=\pi K_{2 n-1}\left(k^{\prime}\right)_{E}
$$

inside $K_{2 n-1}(k)_{E}$ so that we can use the same bases for either side. The same holds for $\tilde{\pi}(E \otimes k)$ and $\pi\left(E \otimes k^{\prime}\right)$ inside $E \otimes k$. Moreover, $E[G] \tilde{\pi}$ is the pullback to $G$ of the $G / N$-representation $E[G / N] \pi$, so that $L(s, E[G] \tilde{\pi}, \mathbb{Q})=L(s, E[G / N] \pi, \mathbb{Q})$ and similarly for the $p$-adic $L$-functions.

(2) If $k$ is a totally real number field $k$ let $\tilde{k}$ be its (totally real) Galois closure over $\mathbb{Q}$. Then $k=\tilde{k}^{H}$ for some subgroup $H$ of $G=\operatorname{Gal}(\tilde{k} / \mathbb{Q}), \pi=|H|^{-1} \sum_{h \in H} h$ is an idempotent in $\mathbb{Q}[G]$, and for $n \geq 2$ odd Conjecture 3.18 for $\pi$ is equivalent to Conjecture 2.17 for $k$. Namely, $K_{2 n-1}(k)_{\mathbb{Q}}=\pi K_{2 n-1}(\tilde{k})_{\mathbb{Q}} \subseteq K_{2 n-1}(\tilde{k})_{\mathbb{Q}}$ by Remark 3.22 and $\pi \tilde{k}=k$ so that we can use the same $\mathbb{Q}$-bases in each case. Moreover, $\mathbb{Q}[G] \pi \cong \operatorname{Ind}_{H}^{G}\left(1_{H}\right)=\mathbb{Q}[G] \otimes_{\mathbb{Q}[H]} 1_{H}$ with $1_{H}$ the trivial 1-dimensional representation of $H$, as one easily sees by mapping $\beta$ in $\mathbb{Q}[G] \pi$ to $\beta \otimes v$ and $\sum_{\sigma} a_{\sigma} \sigma \otimes(\lambda v)$ to $\lambda \sum_{\sigma} a_{\sigma} \sigma \pi,\{v\}$ being a basis of $1_{H}$. By well-known properties of Artin $L$-functions [39, Prop. 10.4(iv)] this implies that $\zeta_{k}(s)=L(s, \mathbb{Q}[G] \pi, \mathbb{Q})$, and similarly for the $p$-adic $L$-functions.

Remark 3.24. We make the relation between Conjecture 3.18(1) and Beilinson's conjecture [2, Conjecture 3.4] for (Artin) motives explicit since the relation 
between the two elements of $E^{*}$ involved also shows up very explicitly in our computer calculations, suggesting that the element for the formulation at $s=1-n$ is simpler than for our formulation at $s=n$ (see Remark 7.8). We provide some details since we could not find a detailed enough reference in the literature.

With notation as in Conjecture 3.18 and (2.6) we have

$$
\begin{aligned}
L\left(1-s, \chi_{\pi}^{\vee} \otimes \mathrm{id}, \mathbb{Q}\right)= & W\left(\chi_{\pi}^{\vee} \otimes \mathrm{id}\right) C\left(\chi_{\pi}^{\vee} \otimes \mathrm{id}\right)^{s-\frac{1}{2}}\left(2(2 \pi)^{-s} \Gamma(s)\right)^{\chi^{\vee}(1)} \\
& \times(\cos (\pi s / 2))^{n^{+}}(\sin (\pi s / 2))^{n^{-}} L\left(s, \chi_{\pi} \otimes \mathrm{id}, \mathbb{Q}\right),
\end{aligned}
$$

with $\chi_{\pi}^{\vee}$ the dual character of $\chi_{\pi}, W\left(\chi_{\pi}^{\vee} \otimes \mathrm{id}\right)$ in $E \otimes \mathbb{C}$, and $C\left(\chi_{\pi}^{\vee} \otimes \mathrm{id}\right)=C\left(\chi_{\pi} \otimes \mathrm{id}\right)$ in $\mathbb{Q}^{*}$. If $m=\operatorname{dim}_{E}(E[G] \pi)$, then $n^{+}=m$ and $n^{-}=0$ for $n$ odd, and $n^{-}=m$ and $n^{+}=0$ for $n$ even. In either case $L\left(s, \chi_{\pi}^{\vee} \otimes \mathrm{id}, \mathbb{Q}\right)$ has a zero of order $m$ at $s=1-n$. Moreover

$$
W\left(\chi_{\pi}^{\vee} \otimes \mathrm{id}\right) C\left(\chi_{\pi}^{\vee} \otimes \mathrm{id}\right)^{1 / 2}=e_{n} i^{m(n-1)} D\left(M_{\pi}^{E}\right)^{1 / 2, \infty}
$$

for some $e_{n}$ in $E^{*}=\left(E \otimes_{\mathbb{Q}} \mathbb{Q}\right)^{*}$ by $[20$, Propositions 5.5 and 6.5$]$ since $D^{1 / 2, \infty}$ can be taken to be the same for $M_{\pi}^{E}$ and the associated determinant representation (cf. [31, p.360]). Hence the first non-vanishing coefficient in the Taylor expansion of $L\left(s, \chi_{\pi}^{\vee} \otimes \mathrm{id}, \mathbb{Q}\right)$ around $s=1-n, L^{\sharp}\left(1-n, \chi_{\pi}^{\vee} \otimes \mathrm{id}, \mathbb{Q}\right)$, equals

$$
\delta_{n} e_{n}(2 \pi i)^{m(1-n)}((n-1) ! / 2)^{m} C\left(\chi_{\pi}^{\vee} \otimes \mathrm{id}\right)^{n-1} D\left(M_{\pi}^{E}\right)^{1 / 2, \infty} L\left(n, \chi_{\pi} \otimes \mathrm{id}, \mathbb{Q}\right)
$$

with $\delta_{n}=(-1)^{m(n-1) / 2}$ when $n$ is odd, and $\delta_{n}=(-1)^{m(n+2) / 2}$ when $n$ is even. In particular, Conjecture 3.18(1) is equivalent with

$$
L^{\sharp}\left(1-n, \chi_{\pi}^{\vee} \otimes \mathrm{id}, \mathbb{Q}\right)=\delta_{n} e_{n}((n-1) ! / 2)^{m} C\left(\chi_{\pi}^{\vee} \otimes \mathrm{id}\right)^{n-1} e\left(n, M_{\pi}^{E}\right) \widetilde{R}_{n, \infty}\left(M_{\pi}^{E}\right)
$$

with the renormalized regulator $\widetilde{R}_{n, \infty}\left(M_{\pi}^{E}\right)=(2 \pi i)^{m(1-n)} R_{n, \infty}\left(M_{\pi}^{E}\right)$.

Let us compare this with Beilinson's conjecture for a motive associated with $\pi$. We associate motives covariantly to smooth projective varieties over $\mathbb{Q}$ as in $[2$, $\S 2.4]$. The Galois group $G$ acts on the left on $k$, hence on the $\operatorname{right}$ on $\operatorname{Spec}(k)$, and we let $\mathcal{M}_{\pi}$ be the motive corresponding to $\pi$ under this action. Then $G$ acts on the left on the cohomology theories on $\operatorname{Spec}(k)$ as well as its $K$-theory, and after tensoring with $E$ the corresponding groups for $\mathcal{M}_{\pi}$ are the images under $\pi$. Thus, the relevant motivic cohomology of $\mathcal{M}_{\pi}$ is $H_{M}^{1}\left(\mathcal{M}_{\pi / \mathbb{Z}}, \mathbb{Q}(n)\right)=K_{2 n-1}\left(M_{\pi}^{E}\right)$. 
We shall need the non-degenerate $E$-bilinear pairing

$$
\begin{aligned}
& E[G] \times \bigoplus_{\tau: k \rightarrow \mathbb{C}} E \rightarrow E \\
& \left(\sum_{\sigma} a_{\sigma} \sigma,\left(b_{\tau}\right)_{\tau}\right) \mapsto \sum_{\sigma} a_{\sigma} b_{\phi_{\infty} \circ \sigma^{-1}} .
\end{aligned}
$$

It factors through the left $E[G]$-action on $\oplus_{\tau} E$ (given by $\sigma\left(\left(b_{\tau}\right)_{\tau}\right)=\left(b_{\tau \circ \sigma^{-1}}\right)_{\tau}$ ), hence is trivial on $E[G](1-\pi) \times \pi\left(\oplus_{\tau} E\right)$ and $E[G] \pi \times(1-\pi)\left(\oplus_{\tau} E\right)$. We therefore obtain a non-degenerate $E$-bilinear pairing

$$
\langle\cdot, \cdot\rangle: E[G] \pi \times \pi\left(\oplus_{\tau} E\right) \rightarrow E
$$

that identifies $E[G] \pi$ and $\pi\left(\oplus_{\tau} E\right)$ as dual $E[G]$-modules.

Tensoring (2.1) with $E$ and applying $\pi$ we get

$$
H_{\mathcal{D}}^{1}\left(\mathcal{M}_{\pi / \mathbb{R}}, \mathbb{R}(n)\right) \cong \pi\left(\bigoplus_{\tau: k \rightarrow \mathbb{C}} E \otimes \mathbb{R}(n-1)\right) .
$$

Note that the left-hand side is a subspace of the right-hand side by (2.1), but because $\operatorname{dim}_{E}\left(K_{2 n-1}\left(M_{\pi}^{E}\right)\right)=\operatorname{dim}_{E}(E[G] \pi)=\operatorname{dim}_{E}\left(\pi\left(\oplus_{\tau} E\right)\right)$ by our assumption on $\pi$ and (3.28), and the regulator map (2.3) tensored with $\mathbb{R}$ is injective, equality must hold. For the Beilinson regulator we therefore have to compare two $E$-structures on $\operatorname{det} H_{\mathcal{D}}^{1}\left(\mathcal{M}_{\pi / \mathbb{R}}, \mathbb{R}(n)\right)$, the first one coming from Betti cohomology,

$$
H_{B}^{0}\left(\mathcal{M}_{\pi / \mathbb{R}}, \mathbb{Q}(n-1)\right) \cong \pi\left(\bigoplus_{\tau: k \rightarrow \mathbb{C}} E \otimes \mathbb{Q}(n-1)\right) \subseteq \pi\left(\bigoplus_{\tau: k \rightarrow \mathbb{C}} E \otimes \mathbb{R}(n-1)\right),
$$

and the second one being induced by the Beilinson regulator map (2.3),

$$
H_{M}^{1}\left(\mathcal{M}_{\pi / \mathbb{R}}, \mathbb{Q}(n)\right) \rightarrow H_{\mathcal{D}}^{1}\left(\mathcal{M}_{\pi / \mathbb{R}}, \mathbb{R}(n)\right)=\pi\left(\bigoplus_{\tau: k \rightarrow \mathbb{C}} E \otimes \mathbb{R}(n-1)\right) .
$$

Choosing an $E$-basis of $\pi\left(\oplus_{\tau} E\right)$, and multiplying it by $(2 \pi i)^{n-1}$ to obtain an $E$-basis of $\pi\left(\oplus_{\tau} E \otimes \mathbb{Q}(n-1)\right)$, it is easy to see that Beilinson's regulator $R_{\text {Bei }}$ for $\mathcal{M}_{\pi}$ satisfies

$$
R_{\mathrm{Bei}}=(2 \pi i)^{m(1-n)} \operatorname{det}[\cdot, \cdot]_{\infty} / \operatorname{det}\langle\cdot, \cdot\rangle=\widetilde{R}_{n, \infty}\left(M_{\pi}^{E}\right) / \operatorname{det}\langle\cdot, \cdot\rangle
$$

where all determinants are computed using the chosen $E$-bases.

Finally, we compare $L$-functions. We have

$$
H_{\mathrm{et}}^{0}\left(\operatorname{Spec}(k) \otimes \overline{\mathbb{Q}}, \mathbb{Q}_{l}\right) \cong \bigoplus_{\tau: k \rightarrow \overline{\mathbb{Q}}} \mathbb{Q}_{l} \cong \mathbb{Q}_{l} \otimes_{\mathbb{Q}}(\underset{\tau: k \rightarrow \overline{\mathbb{Q}}}{\bigoplus} \mathbb{Q})
$$


so that $H_{\text {et }}^{0}\left(\mathcal{M}_{\pi}, \mathbb{Q}_{l}\right) \cong \mathbb{Q}_{l} \otimes \pi\left(\oplus_{\tau} E\right)$ as $\mathbb{Q}_{l} \otimes E[G]$-modules. The $\mathbb{Q}_{l}$ plays no role, and as in (3.28) we see that $\pi\left(\oplus_{\tau: k \rightarrow \overline{\mathbb{Q}}} E\right)$ is dual to $E[G] \pi$ as $E[G]$-module. As the motivic $L$-function uses the geometric rather than the arithmetic Frobenius (cf. $\left[34\right.$, p.26]), we obtain that $L\left(s, \mathcal{M}_{\pi}\right)$ of $[2, \S 3]$ is equal to $L\left(s, \chi_{\pi} \otimes \mathrm{id}, \mathbb{Q}\right)$. If $\theta: E[G] \rightarrow E[G]$ is the $E$-linear involution obtained by replacing each $\sigma$ in $G$ with $\sigma^{-1}$, then we need to consider $\mathcal{M}_{\pi}^{0}=\mathcal{M}_{\theta(\pi)}$ instead of $\mathcal{M}_{\pi}$. But the map $E[G] \pi \times E[G] \theta(\pi) \rightarrow E$ mapping $(\alpha, \beta)$ to the coefficient of the neutral element of $G$ in $\theta(\beta) \alpha$ is easily seen to identify $E[G] \theta(\pi)$ and $E[G] \pi$ as dual $E[G]$-modules, so that $L\left(s, \mathcal{M}_{\pi}^{0}\right)=L\left(s, \chi_{\pi}^{\vee} \otimes \mathrm{id}, \mathbb{Q}\right)$.

\section{More explicit $K$-Groups AND REgulators}

In this section we first describe an inductive procedure that conjecturally gives $K_{2 n-1}(k)_{\mathbb{Q}}(n \geq 2)$ for any number field $k$. It is originally due to Zagier [54], but we essentially give a reformulation by Deligne [21]. We also describe results concerning Conjecture 3.18 when the action of $G$ on $E[G] \pi$ is Abelian.

In order to describe Zagier's conjecture we need the functions

$$
\operatorname{Li}_{n}(z)=\sum_{l \geq 1} \frac{z^{l}}{l^{n}} \quad(n \geq 0)
$$

for $z$ in $\mathbb{C}$ with $|z|<1$ if $n=0$ or 1 , and $|z| \leq 1$ if $n \geq 2$. In particular, $\operatorname{Li}_{1}(z)$ is the main branch of $-\log (1-z)$. Using that $d \operatorname{Li}_{n+1}(z)=\operatorname{Li}_{n}(z) \mathrm{d} \log (z)$ they extend to multi-valued analytic functions on $\mathbb{C} \backslash\{0,1\}$. By simultaneously continuing all $\mathrm{Li}_{n}$ along the same path one obtains single-valued functions on $\mathbb{C} \backslash\{0,1\}$ (see $[54, \S 7]$ or $[19$, Remark 5.2]) by putting

$$
P_{n}(z)=\pi_{n-1}\left(\sum_{j=0}^{n-1} \frac{b_{j}}{j !}(2 \log |z|)^{j} \operatorname{Li}_{n-j}(z)\right) \quad(n \geq 1),
$$

with $b_{j}$ the $j$-th Bernoulli number and $\pi_{n-1}$ the projection of $\mathbb{C}=\mathbb{R}(n-1) \oplus \mathbb{R}(n)$ onto $\mathbb{R}(n-1)$. These functions satisfy $P_{n}(\bar{z})=\overline{P_{n}(z)}$ as well as

$$
P_{n}(z)+(-1)^{n} P_{n}(1 / z)=0
$$

and

$$
P_{n}\left(z^{m}\right)=m^{n-1} \sum_{\zeta^{m}=1} P_{n}(\zeta z)
$$

when $m \geq 1$ and $z^{m} \neq 1$. 
We can now describe the conjecture. For $n \geq 2$ we let $\widetilde{B}_{n}(k)$ be a free Abelian group on generators $[x]_{n}^{\sim}$ with $x \neq 0,1$ in $k$. Define

$$
\begin{aligned}
\widetilde{P}_{n}: \widetilde{B}_{n}(k) & \rightarrow\left(\oplus_{\sigma: k \rightarrow \mathbb{C}} \mathbb{R}(n-1)\right)^{+} \\
{[x]_{n}^{\sim} } & \mapsto\left(P_{n}(\sigma(x))\right)_{\sigma},
\end{aligned}
$$

with $\left(\sigma: \oplus_{k \rightarrow \mathbb{C}} \mathbb{R}(n-1)\right)^{+}=\left\{\left(a_{\sigma}\right)_{\sigma}\right.$ such that $\left.\overline{a_{\sigma}}=a_{\bar{\sigma}}\right\}$. Then we define inductively, for $n \geq 2$,

$$
\mathrm{d}_{n}: \widetilde{B}_{n}(k) \rightarrow\left\{\begin{array}{cr}
\bigwedge_{\mathbb{Z}} k^{*} & \text { if } n=2 \\
B_{n-1}(k) \otimes_{\mathbb{Z}} k^{*} & \text { if } n>2
\end{array}\right.
$$

by

$$
[x]_{n}^{\sim} \mapsto\left\{\begin{array}{c}
(1-x) \wedge x \text { if } n=2 \\
{[x]_{n-1} \otimes x \text { if } n>2,}
\end{array}\right.
$$

where $[x]_{n-1}$ denotes the class of $[x]_{n-1}^{\sim}$ in $B_{n-1}(k)$, which is defined as

$$
B_{n}(k)=\widetilde{B}_{n}(k) / \operatorname{Ker}\left(\mathrm{d}_{n}\right) \cap \operatorname{Ker}\left(\widetilde{P}_{n}\right) .
$$

There are some universal relations, one of which is that $[x]_{n}+(-1)^{n}[1 / x]_{n}=0$, a consequence of (4.3).

Conjecture 4.5. If $n \geq 2$ then

(1) there is an injection

$$
\frac{\operatorname{Ker}\left(\mathrm{d}_{n}\right)}{\operatorname{Ker}\left(\mathrm{d}_{n}\right) \cap \operatorname{Ker}\left(\widetilde{P}_{n}\right)} \rightarrow K_{2 n-1}(k)_{\mathbb{Q}}
$$

with image a finitely generated group of rank equal to $\operatorname{dim}_{\mathbb{Q}}\left(K_{2 n-1}(k)_{\mathbb{Q}}\right)$;

(2) Beilinson's regulator map is essentially given by $\widetilde{P}_{n}$ : the diagram

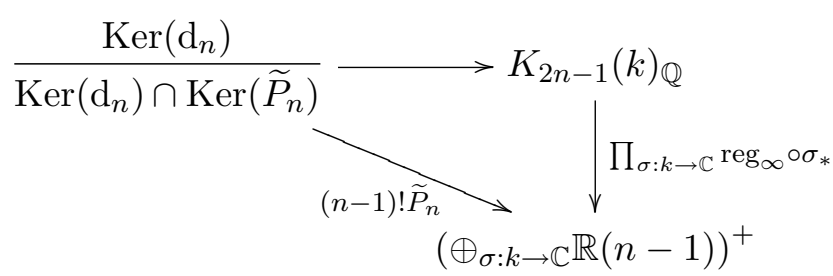

commutes.

Remark 4.6. For $n=2$ the corresponding results were already known before Zagier made his conjecture (see $[9,50]$, as well as $[26, \S 2]$ ). 
From the results in $[19, \S 5]$ one obtains the following.

Theorem 4.7. Let $k$ be a number field and let $n \geq 2$ be an integer. Then there is an injection

$$
\Psi_{n}: \frac{\operatorname{Ker}\left(\mathrm{d}_{n}\right)}{\operatorname{Ker}\left(\mathrm{d}_{n}\right) \cap \operatorname{Ker}\left(\widetilde{P}_{n}\right)} \rightarrow K_{2 n-1}(k)_{\mathbb{Q}}
$$

with finitely generated image, such that the diagram in Conjecture 4.5(2) commutes.

Remark 4.8. The part of Zagier's conjecture that remains open is the question if the rank of the image of $\Psi_{n}$ equals $\operatorname{dim}_{\mathbb{Q}}\left(K_{2 n-1}(k)_{\mathbb{Q}}\right)$. For a cyclotomic field $k$ this holds for any $n \geq 2$ as we shall recall in Example 4.10 below, but it is also known for arbitrary number fields for $n=2$, as mentioned above, or $n=3$ (see [25, §3] and [27, Appendix]).

Remark 4.9. Although not stated explicitly in [19], it is clear from the construction there that the map $\Psi_{n}$ in Theorem 4.7 is natural in $k$. In particular, if $k / \mathbb{Q}$ is Galois then $\operatorname{Gal}(k / \mathbb{Q})$ acts on $\operatorname{Ker}\left(\mathrm{d}_{n}\right) / \operatorname{Ker}\left(\mathrm{d}_{n}\right) \cap \operatorname{Ker}\left(\widetilde{P}_{n}\right)$ (through the obvious action on the generators $\left.[x]_{n}^{\sim}\right)$ as well as on $K_{2 n-1}(k)_{\mathbb{Q}}$, and $\Psi_{n}$ is $\operatorname{Gal}(k / \mathbb{Q})$-equivariant.

Example 4.10. If $k$ is a cyclotomic field then Zagier's conjecture is known in full. By Theorem 4.7 it suffices to see that the rank of the image of $\Psi_{n}$ equals $\operatorname{dim}_{\mathbb{Q}} K_{2 n-1}(k)_{\mathbb{Q}}$. For an $N$-th root of unity $\zeta \neq 1, N[\zeta]_{n}$ is in $\operatorname{Ker}\left(\mathrm{d}_{n}\right) / \operatorname{Ker}\left(\mathrm{d}_{n}\right) \cap$ $\operatorname{Ker}\left(\widetilde{P}_{n}\right)$. If $k=\mathbb{Q}(\zeta)$ for a primitive $N$-th root of unity $\zeta$ with $N>2$ then $\operatorname{dim}_{\mathbb{Q}} K_{2 n-1}(k)_{\mathbb{Q}}=[k: \mathbb{Q}] / 2$ for $n \geq 2$, and the $N\left[\zeta^{j}\right]_{n}$ with $1<j<N / 2$ and $\operatorname{gcd}(j, N)=1$ have $\mathbb{R}$-independent images under $\widetilde{P}_{n}[54, \mathrm{pp} .420-422]$ so that they are $\mathbb{Z}$-independent in $\operatorname{Ker}\left(\mathrm{d}_{n}\right) / \operatorname{Ker}\left(\mathrm{d}_{n}\right) \cap \operatorname{Ker}\left(\widetilde{P}_{n}\right)$, and the same holds for their images under the injective map $\Psi_{n}$. For $k=\mathbb{Q}$ one can easily check directly from Theorem 4.7 and (4.2) that for odd $n \geq 2$ the element $2 \Psi\left([-1]_{n}\right)$ is non-zero.

For the $p$-adic regulator we need to describe the $p$-adic polylogarithms introduced in [16]. We first make a choice of a branch of the $p$-adic logarithm. Recall that a homomorphism $\log _{p}: \mathcal{O}_{\mathbb{C}_{p}}^{*} \rightarrow \mathbb{C}_{p}$ is uniquely determined by the requirement that for $|x|_{p}<1$ it is given by the usual power series for $\log (1+x)$. To extend it to a homomorphism $\log _{p}: \mathbb{C}_{p}{ }^{*} \rightarrow \mathbb{C}_{p}$ it suffices to make an arbitrary choice of $\log _{p}(p)$. Any such extension will be called a branch of the $p$-adic polylogarithm. In what follows we fix one such choice. 
Coleman first produced the $p$-adic polylogarithm out of a more extensive theory of what is now called Coleman integration. For the $p$-adic polylogarithm it is, however, possible to give a more elementary and explicit theory. To do this, consider the class of functions $f: \mathbb{C}_{p} \backslash\{1\} \rightarrow \mathbb{C}_{p}$ that satisfy

(1) for any $a \in \mathbb{C}_{p}$ with $|a-1|_{p}=1$ we have a power series expansion for $f(z)$ in $z-a$ that converges for $|z-a|_{p}<1$;

(2) for $0<|z-1|_{p}<1$ (resp. $|z|_{p}>1$ ) $f(z)$ is given by a polynomial in $\log _{p}(z-1)\left(\right.$ resp. $\left.\log _{p}(1 / z)\right)$ with coefficients that are Laurent series in $z-1$ (resp. $1 / z)$, convergent for $0<|z-1|_{p}<1$ (resp. $|1 / z|_{p}<1$ ).

It is easy to see that differentiation sends this class surjectively onto itself. The $p$ adic polylogarithms are functions $\operatorname{Li}_{n, p}(z)(n \geq 0)$ in this class with the following properties:

(1) $\operatorname{Li}_{0, p}(z)=\frac{z}{1-z}$

(2) $\operatorname{Li}_{n, p}(0)=0$ for $n \geq 0$;

(3) $\mathrm{dLi}_{n+1, p}(z)=\operatorname{Li}_{n, p}(z) \frac{\mathrm{d} z}{z}$ for $n \geq 0$;

(4) for $n \geq 0$ there are power series $g_{n, p}(v)$, convergent for $|v|_{p}<p^{1 /(p-1)}$, such that $g_{n, p}(1 /(1-z))=\operatorname{Li}_{n, p}(z)-\frac{1}{p^{n}} \operatorname{Li}_{n, p}\left(z^{p}\right)$ when $|z-1|_{p}>p^{-1 /(p-1)}$.

Note that $1 / z$ has a singularity at 0 but $\operatorname{Li}_{n, p}(z) / z$ does not, because of the assumption that $\operatorname{Li}_{n, p}(0)=0$. In fact, it is easy to see that $\mathrm{Li}_{n, p}(z)$ is given by the $p$-adically convergent power series (4.1) for $|z|_{p}<1$.

In [6] two of the authors of the present work described an efficient algorithm for the computation of $p$-adic polylogarithms up to a prescribed precision. This will be used in Section 7 to compute the $p$-adic regulator for $n \geq 2$ that we now proceed to describe in terms of suitable combinations of $\log _{p}^{n-m}(z) \operatorname{Li}_{m}(z)$.

By Proposition 6.4 and the correct version of Proposition 6.1 of [16] the $\mathrm{Li}_{n, p}(z)$ for $n \geq 0$ and $z$ in $\mathbb{C}_{p} \backslash\{1\}$ satisfy

$$
\operatorname{Li}_{n, p}(z)+(-1)^{n} \operatorname{Li}_{n, p}(1 / z)=-\frac{1}{n !} \log _{p}^{n}(z)
$$

when $z \neq 0$, and

$$
\operatorname{Li}_{n, p}\left(z^{m}\right)=m^{n-1} \sum_{\zeta^{m}=1} \operatorname{Li}_{n, p}(\zeta z)
$$

when $m \geq 1$ and $z^{m} \neq 1$. 
For $z$ in $\mathbb{C}_{p} \backslash\{0,1\}$ and fixed $n \geq 2$, we define

$$
P_{n, p}(z)=\sum_{j=0}^{n-1} c_{j} \log _{p}^{j}(z) \operatorname{Li}_{n-j, p}(z) \quad(n \geq 2)
$$

for any $c_{j}$ in $\mathbb{C}_{p}$ satisfying $c_{0}=1$ and $\sum_{j=0}^{n-1} c_{j} /(n-j) !=0$, so that $P_{n, p}(z)+$ $(-1)^{n} P_{n, p}(1 / z)=0$. This is the case for $c_{j}=b_{j}$ with $b_{j}$ the $j$-th Bernoulli numbers as before, although the resulting formula is different from (4.2). Another possible natural candidate for the function $P_{n, p}(z)$ is $L_{n, p}(z)+L_{n-1, p}(z) \log _{p}(z) / n$, where

$$
L_{n, p}(z)=\sum_{m=0}^{n-1} \frac{(-1)^{m}}{m !} \log _{p}^{m}(z) \operatorname{Li}_{n-m, p}(z)
$$

(see [5, (1.3) and Remark 1.5]).

The relations corresponding to (4.3) and (4.11) for $P_{n, p}(z)(n \geq 2)$ are then

$$
P_{n, p}(z)+(-1)^{n} P_{n, p}(1 / z)=0
$$

when $z \neq 0,1$, and

$$
P_{n, p}\left(z^{m}\right)=m^{n-1} \sum_{\zeta^{m}=1} P_{n, p}(\zeta z)
$$

when $m \geq 1$ and $z^{m} \neq 0,1$.

For any branch of the $p$-adic logarithm one then has the following result for the $p$-adic regulator map (see [5, Theorem 1.10 or page 909]). Since its formulation depends on the embedding of $k$ we deal with one such embedding at a time.

Theorem 4.14. Let $k$ be a number field, and let $F \subset \overline{\mathbb{Q}}_{p}$ be the topological closure of the Galois closure of any embedding $k \rightarrow \overline{\mathbb{Q}}_{p}$. For $\sigma: k \rightarrow F$ let

$$
\left.B_{n}^{\sigma}(k)=\left\langle[x]_{n}\right| \sigma(x), 1-\sigma(x) \text { are in } \mathcal{O}_{F}^{*}\right\rangle \subseteq B_{n}(k)=\frac{\widetilde{B}_{n}(k)}{\operatorname{Ker}\left(\mathrm{d}_{n}\right) \cap \operatorname{Ker}\left(\widetilde{P}_{n}\right)} .
$$

Then

$$
\begin{aligned}
\widetilde{B}_{n}(k) & \rightarrow F \\
{[x]_{n} } & \mapsto P_{n, p}(\sigma(x))
\end{aligned}
$$

induces a map

$$
P_{n, p}^{\sigma}: B_{n}^{\sigma}(k) \rightarrow F
$$


and the solid arrows in

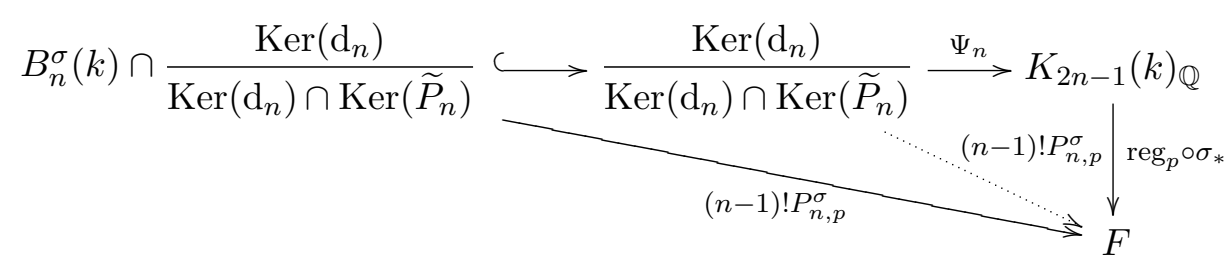

form a commutative diagram.

Remark 4.15. It was conjectured in [5, Conjecture 1.14] that the dotted arrow exists and that the full diagram commutes. This is known to hold for $N[\zeta]_{n}$ if $\zeta$ is any $N$-th root of unity other than 1 (see Theorem 1.12 of loc. cit.).

Remark 4.16. The formulae for the complex and $p$-adic regulators involved in Theorems 4.7 and 4.14 are stated up to sign in [19, Proposition 4.1] (with a normalizing factor) and [5, Theorem 1.10(2) or Proposition 7.14] respectively, since they depend on the choice of $\Psi_{n}$, which is natural only up to sign. In the $p$-adic case it is clear from [5, Proposition 7.10] that the sign is $(-1)^{n}$ if the "relativity isomorphism" in the $p$-adic case is normalized as in Proposition 5.7 of loc. cit. and the $K$-theoretic relativity isomorphism as in (3.1) of loc. cit. corresponds to this under the naturality of the regulator map. In order to see that the sign for the complex regulator is the same with the same choice of $\Psi_{n}$, we observe that one can use the techniques of [5, Appendix A and Section 5] to describe the target of the complex regulator map for $[z]_{n}$ with $z$ in $\mathbb{C} \backslash\{0,1\}$ in [19] by means of a complex with the same formal structure as in [5, Definition 5.2], but using $\mathbb{R}(n-1)$-valued $C^{\infty}$-forms $\psi$ with logarithmic poles on copies of $\left(\mathbb{P}_{\mathbb{C}}^{1} \backslash\{1, z\}\right)^{j}$ indexed by increasing functions $f:[1, \ldots, j] \rightarrow[1, \ldots, n-1]$. Using the maps $\psi \mapsto \int_{\left(\mathbb{P}_{\mathbb{C}}^{1}\right)^{j}} \mathrm{~d} \arg \left(t_{f(1)}\right) \wedge \cdots \wedge \mathrm{d} \arg \left(t_{f(j)}\right) \wedge \psi$ for a $j$-form $\psi$ with index $f$ : $[1, \ldots, j] \rightarrow[1, \ldots, n-1]$, and suitable normalizing factors, one writes down an $\mathbb{R}$-linear map $\Pi_{\infty}$ on such forms, with values in $\mathbb{R}(n-1)$, that vanishes on exact forms and satisfies the analogous normalization condition as $\Pi=\Pi_{p}$ in $[5$, Proposition 5.7]. Moreover, as in [19, Section 2.5] one sees that the subcomplex of forms on the component where $j=n-1$ that vanish if some $t_{l}=0$ or $\infty$ gives a quasi-isomorphism under inclusion, and that the image of the regulator of $[z]_{n}$ is given by $\varepsilon_{n}(z)$ as in Section 4 of loc. cit. (up to sign since one has to use the correct formula for $\omega_{n}$, which can be obtained as in [5, Section 6]). Using the computations on pages 236 and 237 of [19] one then sees that $\Pi_{\infty}$ maps $\varepsilon_{n}(z)$ 
to $(-1)^{n}(n-1) ! P_{n}^{b}(z)$, where $P_{n}^{b}(z)=\pi_{n-1}\left(\sum_{l=0}^{n-1} \frac{1}{l !}(-\log |z|)^{l} \operatorname{Li}_{n-l}(z)\right)$, which induces the same map on $\operatorname{Ker}\left(\mathrm{d}_{n}\right) / \operatorname{Ker}\left(\mathrm{d}_{n}\right) \cap \operatorname{Ker}\left(\widetilde{P}_{n}\right)$ as $(-1)^{n}(n-1) ! P_{n}(z)$. Since the relativity isomorphism in $K$-theory only depends on a choice of $t_{l}=0$ or $\infty$ for $l=1, \ldots, n-1$ this shows that the signs in the formulae for the complex and $p$-adic regulators of $[z]_{n}$ match for the same choice of $\Psi_{n}$, and we adjust $\Psi_{n}$ so that those signs disappear.

We conclude this section by considering Conjecture 3.18 when the action of $G$ on $E[G] \pi$ is Abelian.

Proposition 4.17. Let $N \geq 2, k=\mathbb{Q}\left(\mu_{N}\right), G=\operatorname{Gal}(k / \mathbb{Q})=(\mathbb{Z} / N \mathbb{Z})^{*}$ where a in $(\mathbb{Z} / N \mathbb{Z})^{*}$ corresponds to $\psi_{a}$ in $G$ satisfying $\psi_{a}(\zeta)=\zeta^{a}$ for all $\zeta$ in $\mu_{N}$. Assume that $E$ contains a root of unity of order equal to the exponent of $G$ and let $\pi$ in $E[G]$ be the idempotent corresponding to an irreducible character $\chi$ of G. Then parts (1), (2) and (3) of Conjecture 3.18 hold for $\pi$ and $n \geq 2$ if $\chi(-1)=(-1)^{n-1}$.

Proof. That Conjecture 3.18(1) holds in this case is well-known (cf. [38]) but we make it explicit here for part (3) of the conjecture (cf. [30, Proposition 3.1]).

We deal with the case $N=2$ later so by Remark 3.23(1) we may assume $N>2$ and $\chi$ primitive. The idempotent $\pi$ corresponding to $\chi$ is $|G|^{-1} \sum_{a=1}^{\prime N} \chi^{-1}(a) \otimes \psi_{a}$, where the prime indicates that we sum over $a$ that are relatively prime to $N$.

Note that by our assumption on $\chi(-1)$ and Proposition 3.8 the dimension of $K_{2 n-1}\left(M_{\pi}^{E}\right)$ equals 1. Fixing a generator $\zeta$ of $\mu_{N} \subset k^{*}$ we use $\pi, \pi(1 \otimes \zeta)$ and $\pi\left(1 \otimes[\zeta]_{n}\right)$ as basis vectors of $E[G] \pi, M_{\pi}^{E}$ and $K_{2 n-1}\left(M_{\pi}^{E}\right)$ respectively, where we simplify notation by writing $[\zeta]_{n}$ instead of $\Psi_{n}\left([\zeta]_{n}\right)$ as in Theorem 4.7.

In order to verify (1) in $E \otimes_{\mathbb{Q}} \mathbb{C}$ it suffices by Remark $3.19(2)$ to consider all embeddings $\sigma: E \rightarrow \mathbb{C}$. If $\eta=\phi_{\infty}(\zeta)$ with $\phi_{\infty}$ as in Section 3 then the $\sigma$-component of $D\left(M_{\pi}^{E}\right)^{1 / 2, \infty}$ becomes

$$
D\left(M_{\pi}^{E}\right)_{\sigma}^{1 / 2, \infty}=|G|^{-1} \sum_{a=1}^{N} \chi_{\sigma}^{-1}(a) \eta^{a}=N|G|^{-1}\left(\sum_{a=1}^{N} \chi_{\sigma}^{\prime}(a) \eta^{-a}\right)^{-1}
$$

by [36, (3) on p.84]. Similarly, by Theorem 4.7 the $\sigma$-component of $R_{n, \infty}\left(M_{\pi}^{E}\right)$ is $R_{n, \infty}\left(M_{\pi}^{E}\right)_{\sigma}=(n-1) !|G|^{-1} \sum_{a=1}^{N} \chi_{\sigma}^{-1}(a) P_{n}\left(\eta^{a}\right)=(n-1) !|G|^{-1} \sum_{a=1}^{N} \chi_{\sigma}^{-1}(a) \operatorname{Li}_{n}\left(\eta^{a}\right)$ 
because $P_{n}\left(\eta^{a}\right)=\left(\operatorname{Li}_{n}\left(\eta^{a}\right)+\chi_{\sigma}^{-1}(-1) \operatorname{Li}_{n}\left(\eta^{-a}\right)\right) / 2$ by our assumption on $\chi(-1)$, where $\mathrm{Li}_{n}$ is computed using the power series in (4.1). They relate to the $\sigma$ component of $L(n, \chi, \mathbb{Q})$ via $L\left(n, \chi_{\sigma}, \mathbb{Q}\right)=(n-1) !^{-1} R_{n, \infty}\left(M_{\pi}^{E}\right)_{\sigma} / D\left(M_{\pi}^{E}\right)_{\sigma}^{1 / 2, \infty}$ according to (2) on page 172 of [16] with the correct sign in the exponent of the Gauss sum as used here (cf. [54, p. 421]). Since $L\left(n, \chi_{\sigma}, \mathbb{Q}\right) \neq 0$ this also shows that $\pi\left(1 \otimes[\zeta]_{n}\right)$ gives a basis of $K_{2 n-1}\left(M_{\pi}^{E}\right)$ as claimed.

Similarly, for (2) and (3) we consider all embeddings $\tau: E \rightarrow \overline{\mathbb{Q}}_{p}$. If now $\phi_{p}(\zeta)=\eta_{p}$ then the $\tau$-component of $D\left(M_{\pi}^{E}\right)^{1 / 2, p}$ is $N|G|^{-1}\left(\sum_{a=1}^{\prime N} \chi_{\tau}(a) \eta_{p}^{-a}\right)^{-1}$ and the $\tau$-component of $R_{n, p}\left(M_{\pi}^{E}\right)$ equals $(n-1) !|G|^{-1} \sum_{a=1}^{\prime N} \chi_{\tau}^{-1}(a) P_{n, p}\left(\eta_{p}^{a}\right)$ by Remark 4.15, independent of our assumption on $\chi(-1)$. By (3) on page 172 of [16] we have

$$
L_{p}\left(n, \chi_{\tau} \omega_{p}^{1-n}, \mathbb{Q}\right)=(n-1) !^{-1} \operatorname{Eul}_{p}\left(k, \chi_{\tau}, \mathbb{Q}\right) R_{n, p}\left(M_{\pi}^{E}\right)_{\tau} / D\left(M_{\pi}^{E}\right)_{\tau}^{1 / 2, p} .
$$

Therefore Conjecture 3.18(1)-(3) hold with $e\left(n, M_{\pi}^{E}\right)=e_{p}\left(n, M_{\pi}^{E}\right)$ in $\mathbb{Q}^{*} \subseteq E^{*}$.

Now assume that $N=2$ so that $\chi$ is the trivial character $1, \pi=1$ and $n \geq 2$ is odd. Taking $1,1 \otimes 1$ and $1 \otimes[-1]_{n}$ as bases of $E[G]=E, M_{\pi}^{E}=E \otimes \mathbb{Q}$ and $K_{2 n-1}(\mathbb{Q})_{E}$ we find from Theorem 4.7, (4.1), (4.2) and (4.4) that

$$
L(n, 1, \mathbb{Q})=\zeta(n)=\frac{2^{n-1}}{1-2^{n-1}} P_{n}(-1)=\frac{2^{n-1}}{(n-1) !\left(1-2^{n-1}\right)} R_{n, \infty}\left(M_{1}^{E}\right),
$$

again proving that $[-1]_{n}$ gives a basis for $K_{2 n-1}(\mathbb{Q})_{\mathbb{Q}}$. Coleman proves that

$$
L_{p}\left(n, \omega_{p}^{1-n}, \mathbb{Q}\right)=\left(1-p^{-n}\right) \lim _{x \rightarrow 1}{ }^{\prime} \operatorname{Li}_{n, p}(x)
$$

where the limit is taken in any subfield of $\mathbb{C}_{p}$ that is of finite ramification degree over $\mathbb{Q}_{p}$. But by (4.12) and (4.13) we can rewrite this as

$$
L_{p}\left(n, \omega_{p}^{1-n}, \mathbb{Q}\right)=\frac{\left(1-p^{-n}\right) 2^{n-1}}{1-2^{n-1}} P_{n, p}(-1)=\frac{\left(1-p^{-n}\right) 2^{n-1}}{(n-1) !\left(1-2^{n-1}\right)} R_{n, \infty}^{p}\left(M_{1}\right) .
$$

Remark 4.18. The $\pi$ in the proof of Proposition 4.17 are primitive in the sense of Definition 3.13. So, by Remarks 3.21(4) and 3.23(1), if Conjecture 3.18 applies to $\pi$ in $E[G]$, then parts (1), (2) and (3) of it hold for $\pi$ for any $E$ if the action on $E[G] \pi$ is Abelian. In particular, parts (1) and (2) of Conjecture 2.17 hold for any totally real Abelian number field by Remark 3.21.

Remark 4.19. Computer calculations show that $L_{p}\left(n, \omega_{p}^{1-n}, \mathbb{Q}\right)$ is in $\mathbb{Q}_{p}^{*}$ when $p=2, \ldots, 19$ and $n=2, \ldots, 20$ is odd, verifying Conjecture 2.17(3) in those 
cases. Similarly, with notation and assumptions as in Proposition 4.17, for the 470 primitive characters $\chi$ of $\operatorname{Gal}\left(\mathbb{Q}\left(\mu_{N}\right) / \mathbb{Q}\right)=(\mathbb{Z} / N \mathbb{Z})^{*}$ with $2 \leq N \leq 50$, $L_{p}\left(n, \chi \otimes \omega_{p}^{1-n}, \mathbb{Q}\right)$ lies in $\left(E \otimes \mathbb{Q}_{p}\right)^{*}$ for those values of $p$ and $n=2, \ldots, 20$ whenever $\chi(-1)=(-1)^{n-1}$. Thus Conjecture 3.18(4) also holds in those cases.

\section{Computing $K$-groups in Practice}

Let $n \geq 2$ and let $k$ be a number field. It follows from Theorem 4.7 that Zagier's approach as described in Section 4 can be used to obtain at least some part of $K_{2 n-1}(k)_{\mathbb{Q}}$. Using the notation introduced in Section 4, in order to carry this approach out in practice one starts with two sets $S \subseteq S^{\prime}$ of primes of $\mathcal{O}_{k}$ and considers only $[x]_{n}^{\sim}$ where $x$ is an $S$-unit and $1-x$ an $S^{\prime}$-unit. When we consider several hundred of such $[x]_{n}^{\sim}$ 's (and avoid using both $[x]_{n}^{\sim}$ and $[1 / x]_{n}^{\sim}$ since $[x]_{n}+(-1)^{n}[1 / x]_{n}=0$ as mentioned in Section 4$)$ this method is well suited to computer calculations. The only point in Zagier's approach that cannot be carried out algebraically is to determine which elements in $\operatorname{Ker}\left(\mathrm{d}_{n}\right)$ are actually in $\operatorname{Ker}\left(\mathrm{d}_{n}\right) \cap \operatorname{Ker}\left(\widetilde{P}_{n}\right)$. This is done using standard methods for finding linear relations between the $\widetilde{P}_{n}\left(\alpha_{j}\right)$ 's for a basis $\left\{\alpha_{j}\right\}$ of $\operatorname{Ker}\left(\mathrm{d}_{n}\right)$ with small integral coefficients. Typically, we used about the first 50 decimals after the decimal point of all the polylogarithms of all elements of $k$ involved in the $\alpha_{j}$ embedded into $\mathbb{C}$ in all possible ways. The relations that were found this way were then verified to hold up to at least 30 additional decimal places. As a final check, when we obtained a subgroup of $\operatorname{Ker}\left(\mathrm{d}_{n}\right) / \operatorname{Ker}\left(\mathrm{d}_{n}\right) \cap \operatorname{Ker}\left(\widetilde{P}_{n}\right)$ with the same rank as $K_{2 n-1}(k)$, the number $q$ in (2.4) (but now using the regulator $\widetilde{V}_{n}(k)$ for the resulting subgroup of $K_{2 n-1}(k)_{\mathbb{Q}}$ rather than $V_{n}(k)$; cf. Remark 2.19) was computed with a precision of up to 120 decimals. Its reciprocal appeared to be rational rather convincingly.

For example, for the cubic field $k=\mathbb{Q}[x] /\left(x^{3}-x^{2}-3 x+1\right)$ with discriminant 148, one of the fields in Example 7.1, we find for $\zeta_{k}(n)\left|D_{k}\right|^{1 / 2} / \widetilde{V}_{n}(k)$ the values

$$
\begin{array}{ll}
-1.7531044558071585098612125639152666179693206722 \times 10^{-2} & (n=3) \\
-4.1170685884062518549452525064367732455835167754 \times 10^{-9} & (n=5)
\end{array}
$$

with reciprocals

$$
\begin{array}{ll}
-57.04166666666666666666666666666666666666666666662 & (n=3) \\
-242891265.59999999999999999999999999999999999998 & (n=5) .
\end{array}
$$


As the calculation of a basis of $K_{2 n-1}(k)_{\mathbb{Q}}$, using the method outlined above, tends to take (substantially) longer when $[k: \mathbb{Q}]$, the absolute value of the discriminant of $k$, or $n$ increase, we describe two straightforward methods to reduce this. They, and most of our arguments below, will rely on Remark 3.22.

Method 5.1. If $k$ is an Abelian extension of $\mathbb{Q}$, hence is contained in a cyclotomic field $k^{\prime}$, then we can find $K_{2 n-1}(k)_{\mathbb{Q}}$ by computing $K_{2 n-1}\left(k^{\prime}\right)_{\mathbb{Q}}$ as in Example 4.10 and applying Remark 3.22 (using Remark 4.9).

Since parts (1), (2) and (3) of Conjecture 3.18 are known for all idempotents of $E[G]$ to which it applies if $G$ is Abelian by Remark 3.21 and Proposition 4.17, this will not be used for verifying Conjecture 3.18. Instead, in the cases that we shall consider we rely on the following method in order to find the $K$-groups.

Method 5.2. Let $k / \mathbb{Q}$ be Galois with Galois group $G, E / \mathbb{Q}$ any extension, and $M$ an irreducible $E[G]$-module in $K_{2 n-1}(k)_{E}$. If $H$ is a subgroup of $G$ with $M^{H} \neq 0$ then $M \subseteq E[G] \cdot K_{2 n-1}\left(k^{H}\right)_{\mathbb{Q}}$. If for every irreducible $M$ we can take $H \neq\{e\}$, then we reduce to finding $K_{2 n-1}(l)_{\mathbb{Q}}$ together with the action of $G$ on it inside $K_{2 n-1}(k)_{\mathbb{Q}}$ for proper subfields $l$ of $k$.

We now discuss in Examples 5.3, 5.5 and 5.7 how to use Method 5.2 for certain Galois extensions $k / \mathbb{Q}$. In the decomposition of $K_{2 n-1}(k)_{E}$ according to central idempotents $\pi_{i}$ of $E[G]$ as in Section 3 we concentrate on those primitive central $\pi_{i}$ for which the action on $E[G] \pi_{i}$ is not Abelian and describe corresponding primitive idempotents $\pi$ (as in Definition 3.13(2)) for later use. Due to restrictions on when we can calculate the $p$-adic $L$-functions numerically (see Section 6 ) we only consider $k$ that are Abelian over a quadratic subfield. Of course Proposition 3.12 describes when Conjecture 3.18 applies to $\pi$, but we work out the structure of the $K$-groups and the Galois action in more detail.

Example 5.3. Let $k / \mathbb{Q}$ be an $S_{3}$-extension with quadratic subextension $q$. With $E=\mathbb{Q}$ and $\sigma$ in $G$ of order three, $\pi_{1}=\left(e+\sigma+\sigma^{2}\right) / 3$ and $\pi_{2}=1-\pi_{1}$ are orthogonal central idempotents with $\pi_{2}$ primitive (as in Definition 3.13(2)). In $\mathbb{Q}[G]=\mathbb{Q}[G] \pi_{1} \oplus \mathbb{Q}[G] \pi_{2}$ the first summand consists of Abelian representations of $G$ and the last of two copies of the irreducible 2-dimensional representation $V$ of $G$. A corresponding primitive idempotent for $\pi_{2}$ is $\pi=\pi_{2} \pi_{H}$ for any subgroup $H=\langle\tau\rangle$ of $G$ of order 2 where $\pi_{H}=(e+\tau) / 2$. 
By Remark 3.22 we have $\pi_{1} K_{2 n-1}(k)_{\mathbb{Q}}=K_{2 n-1}(q)_{\mathbb{Q}}$ so that

$$
K_{2 n-1}(k)_{\mathbb{Q}}=K_{2 n-1}(q)_{\mathbb{Q}} \oplus \pi_{2} K_{2 n-1}(k)_{\mathbb{Q}} .
$$

The last summand is a $\mathbb{Q}[G]$-module isomorphic to $V^{t_{n}}$ for some $t_{n} \geq 0$. As $V=\pi V \oplus \tau^{\prime} \pi V$ for any $\tau^{\prime} \neq \tau$ of order 2 , we find, if $c=k^{H}$,

$$
\pi_{2} K_{2 n-1}(k)_{\mathbb{Q}}=\pi_{2} K_{2 n-1}(c)_{\mathbb{Q}} \oplus \tau^{\prime} \pi_{2} K_{2 n-1}(c)_{\mathbb{Q}}
$$

again by Remark 3.22. So we can reduce to the calculation of $K_{2 n-1}(q)_{\mathbb{Q}}$ and $K_{2 n-1}(c)_{\mathbb{Q}}$ together with the action of $G$ on the latter.

A dimension count in (5.4) using Theorem 2.2 determines $t_{n}$. We distinguish two cases.

Case 1. If $q$ is real then $t_{n}=0$ for $n$ even and 2 for $n$ odd.

Case 2. If $q$ is imaginary then $t_{n}=1$.

Conjecture 3.18 applies to $\pi$ when $t_{n}=2$.

Example 5.5. Let $k / \mathbb{Q}$ be a $D_{8}$-extension with $D_{8}=\left\langle\sigma, \tau \mid \sigma^{4}=\tau^{2}=(\sigma \tau)^{2}=e\right\rangle$. We fix an isomorphism $G=\operatorname{Gal}(k / \mathbb{Q}) \cong D_{8}$ and use notation as in Figure 5.1.
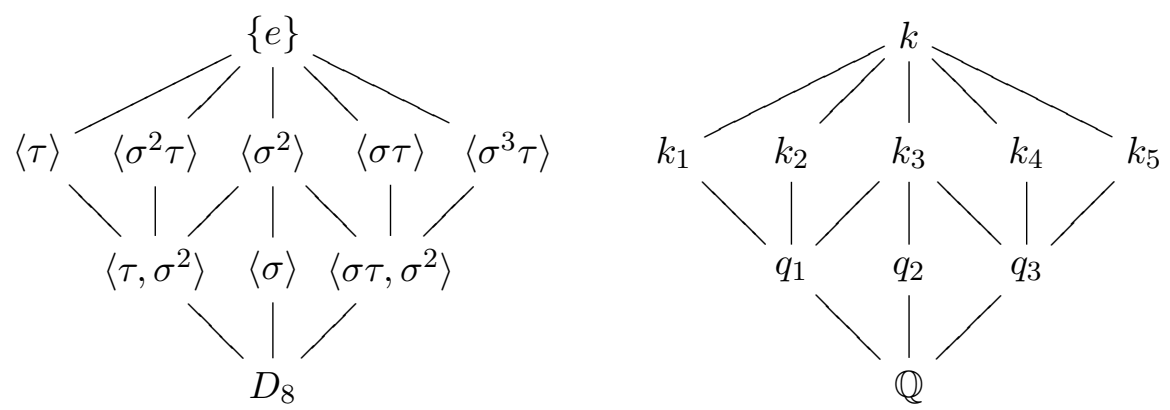

Figure 5.1. The subgroups of $G=D_{8}$ and their fixed fields in $k / \mathbb{Q}$.

Then $\mathbb{Q}[G]=\mathbb{Q}[G] \pi_{1} \oplus \mathbb{Q}[G] \pi_{2}$ where $\pi_{1}=\left(1+\sigma^{2}\right) / 2$ and $\pi_{2}=1-\pi_{1}$ are orthogonal central idempotents. The first term is an Abelian representation of $G$ and the second as a $\mathbb{Q}[G]$-module is isomorphic to $V^{2}$ for $V$ the irreducible 2-dimensional representation of $G$. Then $\pi_{2}$ is a primitive central idempotent and $\pi=\pi_{2} \pi_{H}$ a corresponding primitive idempotent if we let $H=\langle\tau\rangle$ and $\pi_{H}=(1+\tau) / 2$. 
Since $\pi_{1} K_{2 n-1}(k)_{\mathbb{Q}}=K_{2 n-1}\left(k_{3}\right)_{\mathbb{Q}}$ by Remark 3.22 , for $K_{2 n-1}(k)_{\mathbb{Q}}$ we find

$$
K_{2 n-1}(k)_{\mathbb{Q}}=K_{2 n-1}\left(k_{3}\right)_{\mathbb{Q}} \oplus \pi_{2} K_{2 n-1}(k)_{\mathbb{Q}}
$$

with the second term a $\mathbb{Q}[G]$-module isomorphic with $V^{t_{n}}$ for some $t_{n} \geq 0$. If $\tau^{\prime}$ in $G$ has order two and does not commute with $\tau$ then $V=\pi V \oplus \tau^{\prime} \pi V$, so by Remark 3.22 we obtain

$$
K_{2 n-1}(k)_{\mathbb{Q}}=K_{2 n-1}\left(k_{3}\right)_{\mathbb{Q}} \oplus \pi_{2} K_{2 n-1}\left(k_{1}\right)_{\mathbb{Q}} \oplus \tau^{\prime} \pi_{2} K_{2 n-1}\left(k_{1}\right)_{\mathbb{Q}}
$$

and we reduce to the calculation of $K_{2 n-1}\left(k_{3}\right)_{\mathbb{Q}}$ and $K_{2 n-1}\left(k_{1}\right)_{\mathbb{Q}}$ together with the action of $G$ on the latter.

We can find $t_{n}$ by counting dimensions in (5.6), and distinguish three cases.

Case 1. For $k_{3}$ and $k$ totally real $t_{n}=0$ for $n$ even and $t_{n}=2$ for $n$ odd.

Case 2. For $k_{3}$ totally real but $k$ not $t_{n}=2$ for $n$ even and $t_{n}=0$ for $n$ odd.

Case 3. For $k_{3}$ not totally real $t_{n}=1$.

Again Conjecture 3.18 applies to $\pi$ when $t_{n}=2$.

Example 5.7. Let $k / \mathbb{Q}$ be an $S_{3} \times \mathbb{Z} / 3 \mathbb{Z}$-extension, identify $G=\operatorname{Gal}(k / \mathbb{Q})$ and $S_{3} \times \mathbb{Z} / 3 \mathbb{Z}$ via a fixed isomorphism, and choose a generator $\sigma$ of $A_{3} \subset S_{3}$. Let $q$ be the quadratic subfield of $k, s^{\prime}$ the fixed field of $A_{3} \times\{0\}, s^{\prime \prime}$ the fixed field of $\{e\} \times \mathbb{Z} / 3 \mathbb{Z}$ and $s_{i}$ for $i=1,2$ the fixed field of $H_{i}=\langle(\sigma, i)\rangle$.

If $E$ is an extension of $\mathbb{Q}$ that contains a primitive third root of unity $\zeta_{3}$ then

$$
\begin{aligned}
E[G] & =E[G] \pi_{1} \oplus E[G] \pi_{2} \oplus E[G] \pi_{3} \oplus E[G] \pi_{4} \\
& \cong E[\mathbb{Z} / 6 \mathbb{Z}] \oplus W_{1}^{2} \oplus W_{\zeta_{3}}^{2} \oplus W_{\zeta_{3}^{2}}^{2}
\end{aligned}
$$

where

$$
\begin{aligned}
& \pi_{1}=\left((e, 0)+(\sigma, 0)+\left(\sigma^{2}, 0\right)\right) / 3 \\
& \pi_{2}=\left(1-\pi_{1}\right)((e, 0)+(e, 1)+(e, 2)) / 3 \\
& \pi_{3}=\left(1-\pi_{1}\right)\left((e, 0)+\zeta_{3}^{2}(e, 1)+\zeta_{3}(e, 2)\right) / 3 \\
& \pi_{4}=\left(1-\pi_{1}\right)\left((e, 0)+\zeta_{3}(e, 1)+\zeta_{3}^{2}(e, 2)\right) / 3
\end{aligned}
$$

are orthogonal central idempotents of $E[G]$ satisfying $\pi_{1}+\pi_{2}+\pi_{3}+\pi_{4}=(e, 0)$, and $W_{a}$ is the irreducible representation of $G$ given by $(\rho, m)(v)=a^{m} \rho(v)$ for $v$ in the irreducible 2-dimensional representation of $S_{3}$. With $\pi_{H_{i}}=\frac{1}{3} \sum_{h \in H_{i}} h$, a primitive idempotent corresponding to the primitive central idempotent $\pi_{j}$ $(j=3,4)$ is $\pi=\pi_{j} \pi_{H_{i}}$. 
Using Remark 3.22 for $A_{3} \times\{0\}$ and $\{e\} \times \mathbb{Z} / 3 \mathbb{Z}$ we see that

$$
\begin{aligned}
K_{2 n-1}(k)_{E} & =\pi_{1} K_{2 n-1}(k)_{E} \oplus \pi_{2} K_{2 n-1}(k)_{E} \oplus \pi_{3} K_{2 n-1}(k)_{E} \oplus \pi_{4} K_{2 n-1}(k)_{E} \\
& =K_{2 n-1}\left(s^{\prime}\right)_{E} \oplus\left(1-\pi_{1}\right) K_{2 n-1}\left(s^{\prime \prime}\right)_{E} \oplus \pi_{3} K_{2 n-1}(k)_{E} \oplus \pi_{4} K_{2 n-1}(k)_{E} .
\end{aligned}
$$

Because $W_{a}=W_{a}^{H_{1}} \oplus W_{a}^{H_{2}}$ when $a \neq 1$ we have

$$
\pi_{j} K_{2 n-1}(k)_{E}=\pi_{j} K_{2 n-1}\left(s_{1}\right)_{E} \oplus \pi_{j} K_{2 n-1}\left(s_{2}\right)_{E} \quad(j=3,4)
$$

so that we reduce to the calculation of $K_{2 n-1}(s)_{\mathbb{Q}}$ for the sextic subfields $s$ of $k$, together with the action of $G$ when $s=s^{\prime \prime}, s_{1}$ or $s_{2}$.

We have $\pi_{3} K_{2 n-1}(k)_{E} \cong W_{\zeta_{3}}^{t_{n}}$ and $\pi_{4} K_{2 n-1}(k)_{E} \cong W_{\zeta_{3}^{2}}^{t_{n}}$ for the same $t_{n}$ as one sees by taking $E=\mathbb{Q}\left(\zeta_{3}\right)$ and considering the obvious action of $\operatorname{Gal}(E / \mathbb{Q})$ on $E[G]$. Since the dimension of $\left(1-\pi_{1}\right) K_{2 n-1}\left(s^{\prime \prime}\right)_{E}$ was obtained in Example 5.3 we get the following results for $t_{n}$.

Case 1. For $q$ real $t_{n}=0$ when $n$ is even and $t_{n}=2$ when $n$ is odd.

Case 2. For $q$ imaginary $t_{n}=1$.

Conjecture 3.18 applies to $\pi$ corresponding to $\pi_{j}(j=3,4)$ when $t_{n}=2$.

We finish this section with a result that was promised in Remark 3.20(1). Note that the assumption $\operatorname{dim}_{E}(E[G] \pi)=\operatorname{dim}_{E}\left(\pi K_{2 n-1}(k)_{E}\right)$ is not needed here: we consider the regulator map with values in $\pi\left(\oplus_{\tau: k \rightarrow \mathbb{C}} E \otimes \mathbb{R}(n-1)\right)^{+}$ obtained from (2.3), and use Beilinson's regulator $R_{\text {Bei }}$ comparing $E$-structures as in Remark 3.24. Beilinson's conjecture [2, Conjecture 3.4] states that for $L\left(s, \chi_{\pi}^{\vee} \otimes \mathrm{id}, \mathbb{Q}\right)$ the order of vanishing at $s=1-n$ is $\operatorname{dim}_{E}\left(\pi K_{2 n-1}(k)_{E}\right)$ and that the first non-vanishing coefficient of its Taylor expansion at $s=1-n$ equals $e R_{\text {Bei }}$ for some $e$ in $E^{*}$.

Proposition 5.9. Let $k$ be $a G=S_{3}$-extension (resp. $G=D_{8}$-extension) of $\mathbb{Q}$, and let $n \geq 2$. Then this conjecture holds for any primitive idempotent $\pi$ corresponding to $\pi_{2}$ in Example 5.3 (resp. Example 5.5).

Proof. It suffices to prove this when $E=\mathbb{Q}$. If $G=S_{3}$ then by Remark 3.21(3) we may assume $\pi_{H}=\pi_{1} \pi_{H}+\pi$ with $\pi_{1} \pi_{H}$ and $\pi=\pi_{2} \pi_{H}$ orthogonal idempotents, where we use notation as in Example 5.3. The conjecture for $\pi_{H}$ follows from Theorem 2.2 for $c=k^{H}$ by the arguments of Remark 3.23 and the functional equation (2.6) of $\zeta_{c}(s)$. Similarly, it holds for $\pi_{1} \pi_{H}=\frac{1}{6} \sum_{g \in G} g$, where it 
corresponds to that theorem for $k^{G}=\mathbb{Q}$, and it follows that it must hold for $\pi$ as well. If $G=D_{8}$ the argument is identical, using Theorem 2.2 for $k_{1}$ and $q_{1}$.

\section{Computing $p$-Adic $L$-FunCtions}

We briefly sketch the method used to compute values of $p$-adic $L$-functions of 1-dimensional Artin characters. Values of $p$-adic $L$-functions of characters of higher dimension can then be deduced using (2.11). The method we employ is somewhat similar to, but more technical than the construction in [18], and generalizes the one used in [44] to compute values of $p$-adic $L$-functions of real quadratic fields at $s=1$. Note that several modifications are necessary compared to [18] since the construction there is for partial $\zeta$-functions of the ray class group modulo $p$ whereas we need to work with partial $\zeta$-functions of arbitrary ray class groups. Also, we need to replace $p=2$ in a certain number of results by $q=4$. We leave it to the careful reader to check that these changes are indeed possible, or refer to the forthcoming article [43] where the details of the actual method used will be given.

Let $k$ be a totally real number field and let $d=[k: \mathbb{Q}]$. Let $\chi$ be a $\overline{\mathbb{Q}}_{p}$-valued 1-dimensional Artin character of $\operatorname{Gal}(\bar{k} / k)$. As in Section 2, we let $q=p$ if $p$ is odd, and $q=4$ if $p=2$.

By class field theory, $\chi$ can be viewed as a character of a suitable ray class group of $k$. We denote by $\mathfrak{f}$ the conductor of $\chi$, which is an integral ideal of $k$, and we let $\mathfrak{g}$ be the modulus with infinite part $\mathfrak{g}_{\infty}$ consisting of all infinite places of $k$, and finite part $\mathfrak{g}_{0}$ equal to the least common multiple of $\mathfrak{f}$ and $q$. Let $\mathfrak{a}_{1}, \ldots, \mathfrak{a}_{h}$ be representatives of the classes of the ray class group of $k$ modulo $\mathfrak{g}$, and let $\zeta_{\mathfrak{a}_{i}}(s), i=1, \ldots, h$, be the corresponding partial $\zeta$-functions. According to [39, Chapter VII, p.526] we have, for any isomorphism $\sigma: \overline{\mathbb{Q}}_{p} \rightarrow \mathbb{C}$,

$$
\sum_{i=1}^{h} \sigma \circ \chi\left(\mathfrak{a}_{i}\right) \zeta_{\mathfrak{a}_{i}}(s)=\operatorname{Eul}_{p}(s, \sigma \circ \chi, k) L(s, \sigma \circ \chi, k) .
$$

Since all $\zeta_{\mathfrak{a}_{i}}(m)$ are rational for integers $m \leq 0$ by loc. cit. Chapter VII, Corollary 9.9 , we may identify them with $\sigma^{-1}\left(\zeta_{a_{i}}(m)\right)$ in $\mathbb{Q}_{p}$. Thus for such $m$ we have

$$
\sum_{i=1}^{h} \chi\left(\mathfrak{a}_{i}\right) \zeta_{\mathfrak{a}_{i}}(m)=\operatorname{Eul}_{p}^{*}(m, \chi, k) L^{*}(m, \chi, k)
$$


where $L^{*}(m, \chi, k)$ and $\operatorname{Eul}_{p}^{*}(m, \chi, k)$ are as in (2.7) and (2.8).

Now let $\beta \neq 1$ in $\mathcal{O}_{k}$ be such that

(1) $\beta \equiv 1\left(\bmod \mathfrak{g}_{0}\right)$ and $v(\beta)>0$ for every infinite place $v$ of $k$;

(2) $\mathcal{O}_{k} /(\beta) \simeq \mathbb{Z} / b \mathbb{Z}$, where $b=\mathcal{N}(\beta)$ is the norm of the principal ideal $(\beta)$.

Scaling the measures obtained in $\left[18\right.$, Lemme 4.4] for the $\mathfrak{a}_{i}$ we obtain $\mathbb{Q}_{p^{-}}$-valued measures $\widetilde{\lambda}_{i}(i=1, \ldots, d)$ on $\mathbb{Z}_{p}^{d}$, depending on $\beta$ and $\mathfrak{a}_{i}$, such that for all integers $m \leq 0$,

$$
\left(b^{m-1}-1\right) \zeta_{\mathfrak{a}_{i}}(m)=(-1)^{d m} \mathcal{N}\left(\mathfrak{a}_{i}\right)^{-m} \int\left(x_{1} \cdots x_{d}\right)^{-m} d \widetilde{\lambda}_{i} .
$$

The proof of Lemma 4.3 of loc. cit. shows, contrary to the statement of that lemma, that the measures $\widetilde{\lambda}_{i}$ are supported on $\left(-1+q \mathbb{Z}_{p}\right)^{d}$. Therefore pulling back over multiplication by -1 on $\mathbb{Z}_{p}^{d}$ we obtain measures $\lambda_{i}$ on $\mathbb{Z}_{p}^{d}$, supported on $\left(1+q \mathbb{Z}_{p}\right)^{d}$, such that, for the same $m$,

$$
\left(b^{m-1}-1\right) \zeta_{\mathfrak{a}_{i}}(m)=\mathcal{N}\left(\mathfrak{a}_{i}\right)^{-m} \int\left(x_{1} \cdots x_{d}\right)^{-m} d \lambda_{i} .
$$

For a fixed $x$ in $1+q \mathbb{Z}_{p}$ the function $s \mapsto x^{s}$ is defined and analytic on $\mathbb{Z}_{p}$. Since, in general, $\mathcal{N}\left(\mathfrak{a}_{i}\right)$ will not be congruent to 1 modulo $q$, we do the following. For $x$ in $\mathbb{Z}_{p}^{*}$ let $\langle x\rangle=x \omega_{p}(x)^{-1}$ where $\omega_{p}$ is the Teichmüller character on $\mathbb{Z}_{p}^{*}$ (see (2.14)), so that $\langle x\rangle$ is in $1+q \mathbb{Z}_{p}$. Then for any integer $l$ and any integral ideal $\mathfrak{a}$ of $k$ that is coprime to $p$, the function $s \mapsto \omega_{p}(\mathfrak{a})^{l}\langle\mathcal{N}(\mathfrak{a})\rangle^{-s}$ is an analytic $\mathbb{Z}_{p^{-}}$valued function on $\mathbb{Z}_{p}$, whose value equals $\mathcal{N}(\mathfrak{a})^{-m}$ at any integer $m$ with $m+l \equiv 0$ modulo $\varphi(q)$. We define the $p$-adic partial $\zeta$-function of the class of $\mathfrak{a}_{i}$ by mapping $s$ in $\mathbb{Z}_{p} \backslash\{1\}$ to

$$
\zeta_{p, \mathfrak{a}_{i}}(s)=\left(b^{s-1}-1\right)^{-1}\left\langle\mathcal{N}\left(\mathfrak{a}_{i}\right)\right\rangle^{-s} \int^{\prime}\left(x_{1} \cdots x_{d}\right)^{-s} d \lambda_{i},
$$

where the prime indicates that we restrict the domain of integration to $\left(1+q \mathbb{Z}_{p}\right)^{d}$.

Let $\Psi_{i}$ be the function defined by the above integral, that is, for $s$ in $\mathbb{Z}_{p}$,

$$
\Psi_{i}(s)=\int^{\prime}\left(x_{1} \cdots x_{d}\right)^{-s} d \lambda_{i} .
$$

For $a$ in $\mathbb{Z}_{p}$ with $|a-1|_{p} \leq q^{-1}$, and $s$ in $\mathbb{Z}_{p}$, one can write $a^{-s}=\exp _{p}\left(-s \log _{p}(a)\right)$, where $\exp _{p}$ and $\log _{p}$ are the $p$-adic exponential and logarithm functions respectively; and thus the function $s \mapsto a^{-s}$ can be expressed as a power series with coefficients in $\mathbb{Z}_{p}$, where the coefficient of $s^{m}$ has absolute value at most $q^{-m} p^{m /(p-1)}$. 
Developing $\left(x_{1} \cdots x_{d}\right)^{-s}$ in this way as a power series of $s$ and using the fact that the measures $\lambda_{i}$ have bounded norm by [18, Lemme $\left.4.2 \mathrm{bis}\right]$, we see that $\Psi_{i}(s)$ can also be expressed as a power series in $\mathbb{Q}_{p}[[s]]$ where the absolute value of the coefficient of $s^{m}$ is at most $C_{i} q^{-m} p^{m /(p-1)}$ for some $C_{i}>0$. In the same way, the function $s \mapsto\left\langle\mathcal{N}\left(\mathfrak{a}_{i}\right)\right\rangle^{-s}$ can be expressed as a power series in $\mathbb{Z}_{p}[[s]]$ whose coefficients satisfy the same bounds. Similarly, since the only solution for $s$ of $b^{s-1}=1$ with $|s|<q p^{-1 /(p-1)}$ is $s=1$, it follows from the $p$-adic Weierstrass preparation theorem that the function $s \mapsto b^{s-1}-1$ can be expressed as the product of a power of $p$, the polynomial $s-1$, and an invertible power series in $\mathbb{Z}_{p}[[s]]$ with the absolute value of the coefficient of $s^{m}$ bounded by $q^{-m} p^{m /(p-1)}$. Therefore $\zeta_{p, \mathfrak{a}_{i}}(s)$ can be expressed as the quotient of a power series in $\mathbb{Q}_{p}[[s]]$ with the absolute value of the coefficient of $s^{m}$ bounded by $C_{i}^{\prime} q^{-m} p^{m /(p-1)}$ with $C_{i}^{\prime}>0$, and $s-1$.

If we define $p$-adic functions of $s$,

$$
L_{p}^{(l)}(s, \chi, k)=\sum_{i=1}^{h} \omega_{p}\left(\mathfrak{a}_{i}\right)^{l} \chi\left(\mathfrak{a}_{i}\right) \zeta_{p, \mathfrak{a}_{i}}(s) \quad(l \text { modulo } \phi(q)),
$$

then it follows from $(6.1),(6.2)$, and the equality $\zeta_{p, \mathfrak{a}_{i}}(m)=\omega_{p}\left(\mathfrak{a}_{i}\right)^{m} \zeta_{\mathfrak{a}_{i}}(m)$ for any integer $m \leq 0$, that

$$
L_{p}^{(l)}(m, \chi, k)=\operatorname{Eul}_{p}^{*}(m, \chi, k) L^{*}(m, \chi, k)
$$

for all non-positive $m$ such that $l+m \equiv 0$ modulo $\varphi(q)$. In particular, the $p$-adic $L$-function of $\chi$ is given by $L_{p}(s, \chi, k)=L_{p}^{(-1)}(s, \chi, k)$. Moreover, its residue at $s=1$ is zero if $\chi$ is non-trivial because the functions $\omega_{p}\left(\mathfrak{a}_{i}\right)^{-1} \zeta_{p, \mathfrak{a}_{i}}(s)$ all have the same residue there by [18, Corollaire on page 388].

The estimates on the coefficients of the power series expansions above imply the claims in Theorem 2.9(1). Through the $p$-adic Weierstrass preparation theorem they also imply that the function in $(2.11)$ can be written as the quotient of a power series in $\mathbb{Q}_{p}(\chi)[[s]]$ that converges for $s$ in $\mathbb{C}_{p}$ with $|s|<q p^{-1 /(p-1)}$, and a polynomial in $\mathbb{Q}_{p}(\chi)[s]$.

We note in passing that if $l$ is any integer then

$$
L_{p}\left(s, \chi \omega_{p}^{l}, k\right)=\sum_{i=1}^{h} \omega_{p}\left(\mathfrak{a}_{i}\right)^{l-1} \chi\left(\mathfrak{a}_{i}\right) \zeta_{p, \mathfrak{a}_{i}}(s)=L_{p}^{(l-1)}(s, \chi, k),
$$


so that we obtain from (6.4) that

$$
L_{p}\left(m, \chi \omega_{p}^{l}, k\right)=\operatorname{Eul}_{p}^{*}(m, \chi, k) L^{*}(m, \chi, k)
$$

for all $m \leq 0$ with $l+m \equiv 1$ modulo $\varphi(q)$, as stated in Remark 2.13.

In order to compute $L(s, \chi, k)$ for a fixed $s$ in $\mathbb{Z}_{p}$, it suffices to compute $\zeta_{p, \mathfrak{a}_{i}}(s)$, for which one mainly has to compute the $\Psi_{i}(s)$. The measures $\lambda_{i}$ can be represented as power series in $d$ variables,

$$
F_{i}\left(w_{1}, \ldots, w_{d}\right)=\sum_{n_{1}, \ldots, n_{d} \geq 0} a_{i, n_{1}, \ldots, n_{d}} w_{1}^{n_{1}} \cdots w_{d}^{n_{d}},
$$

with (bounded) coefficients

$$
a_{i, n_{1}, \ldots, n_{d}}=\int\left(\begin{array}{l}
x_{1} \\
n_{1}
\end{array}\right) \cdots\left(\begin{array}{l}
x_{d} \\
n_{d}
\end{array}\right) d \lambda_{i}
$$

that can be computed using Shintani's method [48], which is practical for calculations if the base field $k$ is $\mathbb{Q}$ or real quadratic. On the other hand, the function $h$ on $\mathbb{Z}_{p}$ defined by

$$
x \mapsto \begin{cases}x^{-s} & \text { if } x \text { is in } 1+q \mathbb{Z}_{p}, \\ 0 & \text { otherwise }\end{cases}
$$

is continuous and thus admits a Mahler expansion $\sum_{n \geq 0} c_{s, n}\left(\begin{array}{l}x \\ n\end{array}\right)$, where the coefficients satisfy $c_{s, n} \rightarrow 0$ when $n \rightarrow \infty$ and can be easily computed recursively. Then we have

$$
\Psi_{i}(s)=\int h\left(x_{1}\right) \cdots h\left(x_{d}\right) d \lambda_{i}=\sum_{n_{1}, \ldots, n_{d} \geq 0} a_{i, n_{1}, \ldots, n_{d}} c_{s, n_{1}} \cdots c_{s, n_{d}} .
$$

Thus, by computing enough terms in the above sum, we can get a good approximation of the integral, and therefore, by putting everything together, of the $p$-adic $L$-function.

In order to apply this we make (2.10) explicit in the cases we are interested in, writing $L$-functions of representations for the $L$-functions of the corresponding characters. In particular, we write the classical $L$-functions of the irreducible 2-dimensional representations of $S_{3}$ and $D_{8}$ in Examples 5.3 and 5.5, and the irreducible 2-dimensional representations $W_{a}$ with $a \neq 1$ of $S_{3} \times \mathbb{Z} / 3 \mathbb{Z}$ in Example 5.7, as the $L$-functions of Abelian characters over quadratic fields. For later use we also note how to write some of them in terms of $\zeta$-functions of certain fields. 
We take the field of coefficients $E$ to be $\mathbb{C}$ in the remainder of this section, and denote the trivial 1-dimensional representation of any group $G$ by $1_{G}$.

Example 6.5. Let $\mathrm{Alt}_{S_{3}}$ be the 1-dimensional representation of $S_{3}$ through the sign character, and $V$ the irreducible 2-dimensional representation. Since

$$
\operatorname{Ind}_{A_{3}}^{S_{3}} \circ \operatorname{Ind}_{\{e\}}^{A_{3}}\left(1_{\{e\}}\right) \cong \operatorname{Ind}_{\{e\}}^{S_{3}}\left(1_{\{e\}}\right) \cong 1_{S_{3}} \oplus \operatorname{Alt}_{S_{3}} \oplus V^{2}
$$

and $\operatorname{Ind}_{A_{3}}^{S_{3}}\left(1_{A_{3}}\right) \cong 1_{S_{3}} \oplus \operatorname{Alt}_{S_{3}}$ it follows that $\operatorname{Ind}_{A_{3}}^{S_{3}}\left(V^{\prime}\right) \cong V$ for either nontrivial 1-dimensional representation $V^{\prime}$ of $A_{3}$. Applying this to the situation of Example 5.3 we see that $L(s, V, \mathbb{Q})=L\left(s, V^{\prime}, q\right)$. Similarly, if $H$ is any subgroup of $S_{3}$ of order 2 , then $\operatorname{Ind}_{H}^{S_{3}}\left(1_{H}\right) \cong 1_{S_{3}} \oplus V$. In the situation of Example 5.3 this means that $\zeta_{c}(s)=\zeta_{\mathbb{Q}}(s) L(s, V, \mathbb{Q})$.

Example 6.6. If $D_{8}$ is as in Example 5.5, $V_{a, b}$ for $a, b= \pm 1$ is the 1-dimensional representation of $D_{8}$ where $\sigma$ acts as multiplication by $a$ and $\tau$ by $b$, and $V$ is the irreducible 2-dimensional representation of $D_{8}$, then for any subgroup $H$ of $D_{8}$

$$
\operatorname{Ind}_{H}^{D_{8}} \circ \operatorname{Ind}_{\{e\}}^{H}\left(1_{\{e\}}\right) \cong\left(\oplus_{a, b= \pm 1} V_{a, b}\right) \oplus V^{2} .
$$

If $H=\left\langle\tau, \sigma^{2}\right\rangle$ then $\operatorname{Ind}_{\{e\}}^{H}\left(1_{\{e\}}\right) \cong \oplus_{a, b= \pm 1} W_{a, b}$ with $W_{a, b}$ the 1-dimensional representation of $H$ where $\sigma^{2}$ acts as multiplication by $a$ and $\tau$ by $b$. Because $\operatorname{Ind}_{H}^{D_{8}}\left(W_{1, b}\right) \cong \oplus_{a= \pm 1} V_{a, b}$ we have $\operatorname{Ind}_{H}^{D_{8}}\left(W_{-1,1}\right) \cong \operatorname{Ind}_{H}^{D_{8}}\left(W_{-1,-1}\right) \cong V$, so with notation as in Figure 5.1 we get $L(s, V, \mathbb{Q})=L\left(s, W_{-1,1}, q_{1}\right)=L\left(s, W_{-1,-1}, q_{1}\right)$. Similarly $\operatorname{Ind}_{\langle\tau\rangle}^{D_{8}}\left(1_{\langle\tau\rangle}\right) \cong\left(\oplus_{a= \pm 1} V_{a, 1}\right) \oplus V$ so that $\zeta_{k_{1}}(s)=\zeta_{q_{1}}(s) L(s, V, \mathbb{Q})$ as well.

Remark 6.7. If $H=\langle\sigma\rangle$ in Example 6.6 then $\operatorname{Ind}_{\{e\}}^{H}\left(1_{\{e\}}\right) \cong \oplus_{m=1}^{4} U_{i^{m}}$ with $U_{a}$ the 1-dimensional representation of $\langle\sigma\rangle$ where $\sigma$ acts as multiplication by $a$. Now $\operatorname{Ind}_{H}^{D_{8}}\left(U_{a}\right) \cong V_{a, 1} \oplus V_{a,-1}$ when $a= \pm 1$ so that $\operatorname{Ind}_{H}^{D_{8}}\left(U_{a}\right) \cong V$ when $a \neq \pm 1$, and in Example 5.5 we also have $L(s, V, \mathbb{Q})=L\left(s, U_{i}, q_{2}\right)=L\left(s, U_{-i}, q_{2}\right)$.

Example 6.8. If $G=S_{3} \times \mathbb{Z} / 3 \mathbb{Z}$ and $H=A_{3} \times \mathbb{Z} / 3 \mathbb{Z}$ then

$$
\operatorname{Ind}_{H}^{G} \circ \operatorname{Ind}_{\{(e, 0)\}}^{H}\left(1_{\{(e, 0)\}}\right) \cong W \oplus W_{1}^{2} \oplus W_{\zeta_{3}}^{2} \oplus W_{\zeta_{3}^{2}}^{2}
$$

with $W$ the direct sum of the 1-dimensional representations of $G$ and the $W_{a}$ as in Example 5.7. Write $\operatorname{Ind}_{\{(e, 0)\}}^{H}\left(1_{\{(e, 0)\}}\right) \cong \oplus_{a, b} V_{a, b}$ where, for $a$ and $b$ cube roots of unity, $V_{a, b}$ is the 1-dimensional representation of $H$ on which $\left(\sigma^{m}, n\right)$ acts as multiplication by $a^{m} b^{n}$. Since $\operatorname{Ind}_{H}^{G}\left(\oplus_{b} V_{1, b}\right) \cong W$ we find by considering the action of $(e, 1)$ that $\operatorname{Ind}_{H}^{G}\left(V_{a, b}\right) \cong W_{b}$ when $a \neq 1$. In particular, in the notation of Example 5.7, we have $L\left(s, W_{b}, \mathbb{Q}\right)=L\left(s, V_{a, b}, q\right)$ since $q=k^{H}$. 
It is easy to see that the coefficients of $p^{-s}$ in the Euler factors of $L\left(s, W_{\zeta_{3}}, \mathbb{Q}\right)$ and $L\left(s, W_{\zeta_{3}^{2}}, \mathbb{Q}\right)$ are conjugate, but since not all of them can be real it is not possible to express those functions in terms of $\zeta$-functions of subfields of $k$.

\section{EXAMPLES}

In this section we describe the evidence for Conjecture 3.18 in the case of primitive idempotents $\pi$ in $E[\operatorname{Gal}(k / \mathbb{Q})]$ as in Examples 5.3, 5.5 and 5.7, with $E=\mathbb{Q}$ in the first two and $E=\mathbb{Q}\left(\zeta_{3}\right)$ in the last. In the first two cases part (1) of the conjecture is known by Proposition 5.9, but in the last case our evidence for this part is numerical; the same holds for parts (2) through (4) in all cases.

We would like to stress that the statement about the value of the $p$-adic $L$ function in part (4) of the conjecture is actually proved by its numerical verification in all cases that we considered since we can check that an element of $E \otimes \mathbb{Q}_{p}$ is a unit of that ring by computing a close enough approximation of it. The same holds for the statement about the $p$-adic regulator when $n=2$ or 3 since then, in the cases we considered, the calculation in Zagier's conjecture is done purely algebraically so we know we find a subgroup of the $K$-group. (For higher values of $n$ the calculation depends on high-precision calculations of regulators, which doesn't allow us to conclude this.)

For the numerical calculations of the $p$-adic regulator, $R_{n, p}\left(M_{\pi}^{E}\right)$, we assumed that the syntomic regulator map on the subgroup of $K_{2 n-1}(k)_{\mathbb{Q}}$ described in Zagier's Conjecture 4.5 is given by the $\widetilde{P}_{n, p}^{\sigma}$ (as in Remark 4.15). Out of the many possible choices for $P_{n, p}(z)$ as described in (4.12) we used

$$
P_{n, p}(z)=\operatorname{Li}_{n, p}(z)-\log _{p}^{n-1}(z) \operatorname{Li}_{1, p}(z) / n !=\operatorname{Li}_{n, p}(z)+\log _{p}^{n-1}(z) \log _{p}(1-z) / n !
$$

since it is relatively simple from a computational point of view. The calculations of the $P_{n, p}(z)$ for the branch with $\log _{p}(p)=0$ were done in versions 2.11-7, 2.12-19 and 2.12-21 of [51], using an implementation of the algorithm described in [6].

Note that the primitive idempotents in Examples 5.3 and 5.5 are unique up to conjugation in $\mathbb{Q}[G]$ since they all correspond to $\pi_{2}$. But in Example 5.7 replacing $\zeta_{3}$ with $\zeta_{3}^{2}$ changes a primitive idempotent corresponding to $\pi_{3}$ into one corresponding to $\pi_{4}$, and conversely. Because the same holds for the classical and $p$-adic $L$-functions (with values in $E \otimes_{\mathbb{Q}} \mathbb{C}$ and $E \otimes_{\mathbb{Q}} \mathbb{Q}_{p}$ respectively), as well as 
TABLE 1 . Splitting field of $x^{3}-x^{2}-3 x+1$, where $C\left(\chi_{\pi}\right)=2^{2} \cdot 37$.

\begin{tabular}{|c|c|}
\hline$n=3$ & $e\left(3, M_{\pi}\right)^{-1}=2^{-2} \cdot 3^{-2} \cdot 37^{2}$ \\
\hline$p$ & $R_{3, p}\left(M_{\pi}\right) / D\left(M_{\pi}\right)^{1 / 2, p}$ \\
\hline 2 & $(1.00010101001000000010000101111100100010101110100110010110110011000000)_{2} \times 2^{0}$ \\
\hline 3 & $(1.010012121202211021200001102121121100201201100121200211021212)_{3} \times 3^{4}$ \\
\hline 5 & $(4.2320143330214023104113344110103131003140)_{5} \times 5^{6}$ \\
\hline 7 & $(6.354304301412412415450326016336635)_{7} \times 7^{6}$ \\
\hline 11 & $(2.62161235 A 928 A 3423563 A 7888 A)_{11} \times 11^{6}$ \\
\hline$p$ & $L_{p}\left(3, \chi_{\pi} \omega_{p}^{-2}, \mathbb{Q}\right)$ \\
\hline 2 & $(1.00000010100111000100001100100011111010110000101001101110011000101000)_{2} \times 2^{2}$ \\
\hline 3 & $(1.1202122002221002110112100012002001211111011101010220)_{3} \times 3^{0}$ \\
\hline 5 & $(4.11040024440232442233024131040014140)_{5} \times 5^{0}$ \\
\hline 7 & $(5.23516363226501261362543533110)_{7} \times 7^{0}$ \\
\hline 11 & $(A .9542728 A 692401225487 A 278)_{11} \times 11^{0}$ \\
\hline$n=5$ & $e\left(5, M_{\pi}\right)^{-1}=2^{4} \cdot 3^{2} \cdot 5^{-2} \cdot 37^{4}$ \\
\hline$p$ & $R_{5, p}\left(M_{\pi}\right) / D\left(M_{\pi}\right)^{1 / 2, p}$ \\
\hline 2 & $(1.00001010110110011101000011101001000001100000100010000011011110011101)_{2} \times 2^{6}$ \\
\hline 3 & $(1.200111022020102011202110020222210102001020101122110122022)_{3} \times 3^{12}$ \\
\hline 5 & $(3.214210342400224303122204142313310423002)_{5} \times 5^{8}$ \\
\hline 7 & $(1.2103004405355450123124426604231)_{7} \times 7^{10}$ \\
\hline 11 & $(1.2069 A A 607609242834655465)_{11} \times 11^{10}$ \\
\hline$p$ & $L_{p}\left(5, \chi_{\pi} \omega_{p}^{-4}, \mathbb{Q}\right)$ \\
\hline 2 & $(1.00000101111001110001111000010111110100101010001010001001100111100011)_{2} \times 2^{2}$ \\
\hline 3 & $(1.2022122002112102001110211120022002211212022201001210)_{3} \times 3^{0}$ \\
\hline 5 & $(3.34404422113121141420412040113130342)_{5} \times 5^{0}$ \\
\hline 7 & $(4.66001565513622316645262615350)_{7} \times 7^{0}$ \\
\hline 11 & $(1.1 A 26918431 A 09200099 A 81 A 7)_{11} \times 11^{0}$ \\
\hline
\end{tabular}

the Euler factor for $p$, the conjectures for primitive idempotents corresponding to $\pi_{3}$ and $\pi_{4}$ are actually equivalent.

In Examples 7.1, 7.4 and 7.5 we have $E=\mathbb{Q}$ so that we identify $E \otimes_{\mathbb{Q}} \mathbb{C}$ with $\mathbb{C}$, etc., and use notation as in Remark 3.17.

Example 7.1. Let $k$ be a totally real $S_{3}$-extension of $\mathbb{Q}$. Use notation as in Case 1 of Example 5.3, and let $\pi$ be the primitive idempotent corresponding to $\pi_{2}$ of that example. Since $E=\mathbb{Q}$ we shall write $M_{\pi}$ instead of $M_{\pi}^{E}$ in all notation referring to Conjecture 3.18 .

Since $\pi K_{2 n-1}(k)_{\mathbb{Q}}=\pi_{2} K_{2 n-1}(c)_{\mathbb{Q}}$ by Remark 3.22 we computed the $K$-theory of $c$ using the (numerical) methods of Section 5 and tried to recognize the number

$$
e\left(n, M_{\pi}\right)=L\left(n, \chi_{\pi}, \mathbb{Q}\right) \cdot D\left(M_{\pi}\right)^{1 / 2, \infty} / R_{n, \infty}\left(M_{\pi}\right)
$$


TABLE 2. Splitting field of $x^{3}-6 x-2$, where $C\left(\chi_{\pi}\right)=2^{2} \cdot 3^{3} \cdot 7$.

\begin{tabular}{|c|c|}
\hline$n=3$ & $e\left(3, M_{\pi}\right)^{-1}=-2^{-2} \cdot 3^{4} \cdot 7^{2}$ \\
\hline$p$ & $R_{3, p}\left(M_{\pi}\right) / D\left(M_{\pi}\right)^{1 / 2, p}$ \\
\hline 2 & $(1.10000001110000110101010101111101011001000101111010100001000101111010)_{2} \times 2^{0}$ \\
\hline 3 & $(2.1122020011220001210112202100011222122000102121211020022012)_{3} \times 3^{4}$ \\
\hline 5 & $(3.4232302100220101433222440213432224202143)_{5} \times 5^{6}$ \\
\hline 7 & $(4.2544363600510010013523314315122)_{7} \times 7^{7}$ \\
\hline 11 & $(5.481569541633 A 6875525 A 00911)_{11} \times 11^{6}$ \\
\hline$p$ & $L_{p}\left(3, \chi_{\pi} \omega_{p}^{-2}, \mathbb{Q}\right)$ \\
\hline 2 & $(1.01111101110011001100110111100111100010100000100110111100101110110010)_{2} \times 2^{2}$ \\
\hline 3 & $(1.1211000101210110112021221022022211101101102021011220)_{3} \times 3^{0}$ \\
\hline 5 & $(2.13000020122302011221412322140400441)_{5} \times 5^{0}$ \\
\hline 7 & $(4.55510152416245405156545556000)_{7} \times 7^{2}$ \\
\hline 11 & $(1.14 A 21577033 A 19227344806 A)_{11} \times 11^{0}$ \\
\hline$n=5$ & $e\left(5, M_{\pi}\right)^{-1}=-2^{4} \cdot 3^{14} \cdot 5^{-2} \cdot 7^{4}$ \\
\hline$p$ & $R_{5, p}\left(M_{\pi}\right) / D\left(M_{\pi}\right)^{1 / 2, p}$ \\
\hline 2 & $(1.10101101100000101100101100100110101101111111000110011100110000011111)_{2} \times 2^{6}$ \\
\hline 3 & $(2.120122222011210012110212011212210211022212221020022201)_{3} \times 3^{14}$ \\
\hline 5 & $(4.040210142044334334412003042243311012202)_{5} \times 5^{8}$ \\
\hline 7 & $(3.2403415331415026502116243110353)_{7} \times 7^{9}$ \\
\hline 11 & $(7.160964096503643537518492)_{11} \times 11^{10}$ \\
\hline$p$ & $L_{p}\left(5, \chi_{\pi} \omega_{p}^{-4}, \mathbb{Q}\right)$ \\
\hline 2 & $(1.01010111000010111011111101010101111011001011010111110100101100010111)_{2} \times 2^{2}$ \\
\hline 3 & $(1.2002000121100001212101001020022211022201122100111000)_{3} \times 3^{0}$ \\
\hline 5 & $(4.44311102333442121212402410333144343)_{5} \times 5^{0}$ \\
\hline 7 & $(3.41526030363465662002262442000)_{7} \times 7^{0}$ \\
\hline 11 & $(2.687349321 A 27130485753530)_{11} \times 11^{0}$ \\
\hline
\end{tabular}

for $n=3$ and 5 as an element of $\mathbb{Q}^{*}$ by employing the same methods as described in Section 5 for number fields, where $L\left(n, \chi_{\pi}, \mathbb{Q}\right)$ was computed as $\zeta_{c}(n) / \zeta_{\mathbb{Q}}(n)$ (see Example 6.5) using Pari-GP [52]. We succeeded in all cases, and then verified numerically for $p=2,3,5,7$ and 11 if $L_{p}\left(n, \chi_{\pi} \omega_{p}^{1-n}, \mathbb{Q}\right) \neq 0$ and

$$
e\left(n, M_{\pi}\right) \cdot \operatorname{Eul}_{p}\left(n, \chi_{\pi}, \mathbb{Q}\right) \cdot \frac{R_{n, p}\left(M_{\pi}\right)}{D\left(M_{\pi}\right)^{1 / 2, p}} \cdot L_{p}\left(n, \chi_{\pi} \omega_{p}^{1-n}, \mathbb{Q}\right)^{-1}
$$

in $\mathbb{Q}_{p}^{*}$ was equal to 1 .

We put our results for the four totally real $S_{3}$-extensions $k$ of $\mathbb{Q}$ with smallest discriminants in Tables 1 through 4 . Note that $R_{n, p}\left(M_{\pi}\right) / D\left(M_{\pi}\right)^{1 / 2, p}$ is in $\mathbb{Q}_{p}$ by Lemma 3.7 and the same holds for $L_{p}\left(n, \chi_{\pi} \omega_{p}^{1-n}, \mathbb{Q}\right)$ by Lemma 3.15 , so that (approximations of) those numbers can be easily represented in our tables. We 
TABLE 3. Splitting field of $x^{3}-4 x-1$, where $C\left(\chi_{\pi}\right)=229$.

\begin{tabular}{|c|c|}
\hline$n=3$ & $e\left(3, M_{\pi}\right)^{-1}=-2^{-9} \cdot 3^{-2} \cdot 229^{2}$ \\
\hline$p$ & $R_{3, p}\left(M_{\pi}\right) / D\left(M_{\pi}\right)^{1 / 2, p}$ \\
\hline 2 & $(1.01110100101010000001111100101001000110110101000001001011100111100010)_{2} \times 2^{0}$ \\
\hline 3 & $(2.012012211010121011021101220021012212100110002220200211021221)_{3} \times 3^{4}$ \\
\hline 5 & $(4.3433241433301132322003123241441021322342)_{5} \times 5^{6}$ \\
\hline 7 & $(6.262401634562505353633061004510116)_{7} \times 7^{6}$ \\
\hline 11 & $(3.6717 A 20401481085265342054)_{11} \times 11^{7}$ \\
\hline$p$ & $L_{p}\left(3, \chi_{\pi} \omega_{p}^{-2}, \mathbb{Q}\right)$ \\
\hline 2 & $(1.01100001011001101110100010111011001000000110000010000100110111111101)_{2} \times 2^{3}$ \\
\hline 3 & $(2.2120211202111122100220011211121110002020101200021220)_{3} \times 3^{0}$ \\
\hline 5 & $(3.03414332241433441404241012442104412)_{5} \times 5^{0}$ \\
\hline 7 & $(3.24033044132523034433054506150)_{7} \times 7^{0}$ \\
\hline 11 & $(9.21 A 61 A 480 A 10801760893710)_{11} \times 11^{1}$ \\
\hline$n=5$ & $e\left(5, M_{\pi}\right)^{-1}=-2^{-8} \cdot 3^{6} \cdot 11 \cdot 229^{4}$ \\
\hline$p$ & $R_{5, p}\left(M_{\pi}\right) / D\left(M_{\pi}\right)^{1 / 2, p}$ \\
\hline 2 & $(1.11110110101010011011010001011100111001101011001101110111000000010001)_{2} \times 2^{5}$ \\
\hline 3 & $(2.222112021012010001201220011021022211112022112001020100)_{3} \times 3^{16}$ \\
\hline 5 & $(4.3414223144333221124000410221113420102)_{5} \times 5^{10}$ \\
\hline 7 & $(2.5612036605646266635264251053110)_{7} \times 7^{10}$ \\
\hline 11 & $(8.5 A 9220278400758 A 0193 A A 6)_{11} \times 11^{11}$ \\
\hline$p$ & $L_{p}\left(5, \chi_{\pi} \omega_{p}^{-4}, \mathbb{Q}\right)$ \\
\hline 2 & $(1.01010000111010100010011010011100110010001101000101101100101100100011)_{2} \times 2^{3}$ \\
\hline 3 & $(2.1121000022222000210222202101202022011021200021010220)_{3} \times 3^{0}$ \\
\hline 5 & $(4.42011132342110332341130324344440134)_{5} \times 5^{0}$ \\
\hline 7 & $(1.25032245164656402236525435500)_{7} \times 7^{0}$ \\
\hline 11 & $(5.375705346 A A 0810959728302)_{11} \times 11^{0}$ \\
\hline
\end{tabular}

have denoted an element $\left(a_{0}+a_{1} p+a_{2} p^{2}+\cdots\right) \times p^{s}$ in $\mathbb{Q}_{p}^{*}$ with all $a_{j}$ in $\{0, \ldots, p-1\}$ and $a_{0} \neq 0$ by $\left(a_{0} \cdot a_{1} a_{2} \cdots\right)_{p} \times p^{s}$, writing $A$ to represent 10 when $p=11$.

For the $p$-adic regulators we computed each $P_{n, p}(z)$ up to $O\left(p^{L(p)}\right)$ with $p^{L(p)}$ approximately equal to $10^{30}$. The relevant part of $R_{n, p}\left(M_{\pi}\right) / D\left(M_{\pi}\right)^{1 / 2, p}$ has been given in the tables whenever it fitted. For the values of the $p$-adic $L$ function we can only prove that the relative error is at most $1+O\left(p^{M(p)}\right)$, where $M(2)=72, M(3)=47, M(5)=32, M(7)=26$ and $M(11)=22$. This proves that $L_{p}\left(n, \chi_{\pi} \omega_{p}^{1-n}, \mathbb{Q}\right)$ does not vanish; together with the verification that $(7.2)$ equals 1 numerically this proves part (4) of Conjecture 3.18 when $n=3$ and makes it very plausible when $n=5$. The value that we found for (7.3) was $1+O\left(p^{N(p)}\right)$ with $N(2) \geq 82, N(3) \geq 52, N(5) \geq 36, N(7) \geq 27$ and $N(11) \geq 24$, giving numerical evidence for parts (2) and (3) of the conjecture, but also suggesting 
TABLE 4 . Splitting field of $x^{3}-6 x^{2}+2$, where $C\left(\chi_{\pi}\right)=2^{2} \cdot 3^{4} \cdot 5$.

\begin{tabular}{|c|c|}
\hline$n=3$ & $e\left(3, M_{\pi}\right)^{-1}=2^{-2} \cdot 3^{6} \cdot 5$ \\
\hline$p$ & $R_{3, p}\left(M_{\pi}\right) / D\left(M_{\pi}\right)^{1 / 2, p}$ \\
\hline 2 & $(1.11110010110000001011011110010111010011100101111011011000111111110001)_{2} \times 2^{0}$ \\
\hline 3 & $(2.120020100000012102010221000201210120200202222221111002100)_{3} \times 3^{6}$ \\
\hline 5 & $(3.3432222440014423344440441124111231400414)_{5} \times 5^{4}$ \\
\hline 7 & $(5.611651266622656555064166304513241)_{7} \times 7^{6}$ \\
\hline 11 & $(2.805 A 8344265760 A 69 A 2490828 A)_{11} \times 11^{6}$ \\
\hline$p$ & $L_{p}\left(3, \chi_{\pi} \omega_{p}^{-2}, \mathbb{Q}\right)$ \\
\hline 2 & $(1.10100000100110111110111110101101001001110101011111100111101100001111)_{2} \times 2^{2}$ \\
\hline 3 & $(1.1120211121012011012202202010100211210110122222201210)_{3} \times 3^{0}$ \\
\hline 5 & $(2.13242313233011201133333143412022134)_{5} \times 5^{0}$ \\
\hline 7 & $(3.00123442142613541412624063510)_{7} \times 7^{0}$ \\
\hline 11 & $(2.2725 A 17844929880 A 8412281) 11 \times 11^{0}$ \\
\hline$n=5$ & $e\left(5, M_{\pi}\right)^{-1}=-2^{5} \cdot 3^{18} \cdot 5^{2} \cdot 324762301$ \\
\hline$p$ & $R_{5, p}\left(M_{\pi}\right) / D\left(M_{\pi}\right)^{1 / 2, p}$ \\
\hline 2 & $(1.00010100011011001110000011110010000100001101100111101000101001001110)_{2} \times 2^{7}$ \\
\hline 3 & $(1.0200220011011111211112101212120212201200221122102001)_{3} \times 3^{18}$ \\
\hline 5 & $(1.321100221113142104412032003401031323110)_{5} \times 5^{7}$ \\
\hline 7 & $(3.5553063665305405650105042513546)_{7} \times 7^{10}$ \\
\hline 11 & $(A .568607725325302 A 5663 A 746)_{11} \times 11^{10}$ \\
\hline$p$ & $L_{p}\left(5, \chi_{\pi} \omega_{p}^{-4}, \mathbb{Q}\right)$ \\
\hline 2 & $(1.10111101010011001111001000001111111000110110101100010100000000001010)_{2} \times 2^{2}$ \\
\hline 3 & $(1.0002101112212121121001002201100122111211210111102110)_{3} \times 3^{0}$ \\
\hline 5 & $(2.30334130213120322410240113023441000)_{5} \times 5^{0}$ \\
\hline 7 & $(4.62042101122332630656130062420)_{7} \times 7^{0}$ \\
\hline 11 & $(5.491153185997 A 48326862 A 46)_{11} \times 11^{0}$ \\
\hline
\end{tabular}

that the relative precision of the value of the $p$-adic $L$-function is slightly higher than we can prove, justifying the higher precision given in the tables.

Similar calculations were carried out for the next four such $S_{3}$ extensions and the same primes, with very similar results.

The other examples proceed mostly along the same lines as Example 7.1. The values of $L\left(n, \chi_{\pi}, \mathbb{Q}\right)$ in Examples 7.4 and 7.5 below were computed using the formula at the end of Example 6.6, but in Example 7.6 the values of $L\left(n, \chi_{\pi}^{E}, \mathbb{Q}\right)$, with $E=\mathbb{Q}\left(\zeta_{3}\right)$ for $\zeta_{3}$ a primitive cube root of unity, were computed using the algorithms described in [24] and its associated program. In Examples 7.4 and 7.5 we determined if $e\left(n, \chi_{\pi}\right)$ (as in $(7.2)$ ) was in $\mathbb{Q}^{*}$ using the methods of Section 5 , and for Example 7.6 we wrote $e\left(n, M_{\pi}^{E}\right)^{-1}=1 \otimes a+\zeta_{3} \otimes b$ in $E \otimes_{\mathbb{Q}} \mathbb{R}$ and then recognized $a$ and $b$ as integers divided by products of some relatively small primes. 
TABLE 5. Splitting field of $x^{4}-x^{3}-3 x^{2}+x+1$, where $C\left(\chi_{\pi}\right)=5 \cdot 29$.

\begin{tabular}{|c|c|}
\hline$n=3$ & $e\left(3, M_{\pi}\right)^{-1}=2^{-6} \cdot 3^{-1} \cdot 29^{2}$ \\
\hline$p$ & $R_{3, p}\left(M_{\pi}\right) / D\left(M_{\pi}\right)^{1 / 2, p}$ \\
\hline 2 & $(1.11111010111100011100011000110000111000001111111101101000111001001001)_{2} \times 2^{2}$ \\
\hline 3 & $(1.201220211120010110010200120221012101101121011201212202210212)_{3} \times 3^{5}$ \\
\hline 5 & $(2.21221421224100432231134432324224211113143)_{5} \times 5^{3}$ \\
\hline 7 & $(2.260524146626624605001621634435032)_{7} \times 7^{6}$ \\
\hline 11 & $(2.74697855444977862531 A 25026)_{11} \times 11^{6}$ \\
\hline$p$ & $L_{p}\left(3, \chi_{\pi} \omega_{p}^{-2}, \mathbb{Q}\right)$ \\
\hline 2 & $(1.01010010100111000101001000111001101011100000001100110101111010001100)_{2} \times 2^{2}$ \\
\hline 3 & $(1.1011210202202201201020222100121100002100020220220021)_{3} \times 3^{0}$ \\
\hline 5 & $(4.30210324423422014142420103340302330)_{5} \times 5^{0}$ \\
\hline 7 & $(1.16355063143251352506540201034)_{7} \times 7^{0}$ \\
\hline 11 & $(9.9 A 7720 A 981020 A 9 A 32071790) 11 \times 11^{0}$ \\
\hline$n=5$ & $e\left(5, M_{\pi}\right)^{-1}=2^{-4} \cdot 3^{2} \cdot 5^{2} \cdot 29^{4}$ \\
\hline$p$ & $R_{5, p}\left(M_{\pi}\right) / D\left(M_{\pi}\right)^{1 / 2, p}$ \\
\hline 2 & $(1.01101101001101100011000000000011101110110010001010001100111111101001)_{2} \times 2^{8}$ \\
\hline 3 & $(1.0002011102111201211120012012011221112210212111112002202122)_{3} \times 3^{12}$ \\
\hline 5 & $(1.030204001130134123130241412102302410321)_{5} \times 5^{7}$ \\
\hline 7 & $(5.6213331545344431613600254300115)_{7} \times 7^{10}$ \\
\hline 11 & $(9.98414459608789 A 37989 A A 86)_{11} \times 11^{10}$ \\
\hline$p$ & $L_{p}\left(5, \chi_{\pi} \omega_{p}^{-4}, \mathbb{Q}\right)$ \\
\hline 2 & $(1.01111100101100101110101001110010110011011010001011101111000000111001)_{2} \times 2^{2}$ \\
\hline 3 & $(1.1111012010001010110000200000021011010010220212100211)_{3} \times 3^{0}$ \\
\hline 5 & $(4.01341421411140002024312030010110013)_{5} \times 5^{0}$ \\
\hline 7 & $(4.10032134632553222245313526015)_{7} \times 7^{0}$ \\
\hline 11 & $(8.6637671480756 A 9916284377)_{11} \times 11^{0}$ \\
\hline
\end{tabular}

In each case we then verified for $p=2, \ldots, 11$ if (7.3) (or its equivalent) was equal to 1 in $\mathbb{Q}_{p}$ or, for Example 7.6, in $\mathbb{Q}\left(\zeta_{3}\right) \otimes_{\mathbb{Q}} \mathbb{Q}_{p}$. The precision for $P_{n, p}(z)$ and the $p$-adic $L$-functions in Examples 7.4 and 7.5 was as in Example 7.1. In Example 7.6 all $P_{n, p}(z)$ were computed up to $O\left(p^{L(p)}\right)$ with $p^{L(p)}$ approximately $10^{16}$ since in this case the check if the equivalent of $(7.3)$ is 1 is done in $\mathbb{Q}\left(\zeta_{3}\right) \otimes_{\mathbb{Q}} \mathbb{Q}_{p}$ so two coefficients are checked; the values of the $p$-adic $L$-function in this case, which also lie in $\mathbb{Q}\left(\zeta_{3}\right) \otimes \mathbb{Q} \mathbb{Q}_{p}$, were determined up to multiplication by $1 \otimes\left(1+O\left(p^{M(p)}\right)\right)$ with $M(p)$ as in Example 7.1.

Example 7.4. Let $k$ be a totally real $D_{8}$-extension of $\mathbb{Q}$ as in Case 1 of Example 5.5, and use notation as in that example. We again write $M_{\pi}$ instead of $M_{\pi}^{E}$ since $E=\mathbb{Q}$ and consider odd $n \geq 2$. Because $\pi K_{2 n-1}(k)_{\mathbb{Q}}=\pi_{2} K_{2 n-1}\left(k_{1}\right)_{\mathbb{Q}}$ we computed $K_{2 n-1}\left(k_{1}\right)_{\mathbb{Q}}$ numerically using the methods of Section 4 . Our results with $n=3$ and 5 for the first four such extensions when ordered according to 
TABLE 6 . Splitting field of $x^{4}-2 x^{3}-3 x^{2}+4 x+1$, where $C\left(\chi_{\pi}\right)=2^{2} \cdot 3^{2} \cdot 11$.

\begin{tabular}{|c|c|}
\hline$n=3$ & $e\left(3, M_{\pi}\right)^{-1}=2^{-2} \cdot 3^{2} \cdot 11^{2}$ \\
\hline$p$ & $R_{3, p}\left(M_{\pi}\right) / D\left(M_{\pi}\right)^{1 / 2, p}$ \\
\hline 2 & $(1.11001111111100110011011000011011110100000010001100010000111000001110)_{2} \times 2^{5}$ \\
\hline 3 & $(1.101222112200111120002122200210022002021121200101112022212121)_{3} \times 3^{2}$ \\
\hline 5 & $(1.3213042203212141302102134014142431100344)_{5} \times 5^{6}$ \\
\hline 7 & $(1.444434205533111532005253505615656)_{7} \times 7^{6}$ \\
\hline 11 & $(9.61613555149153993505670160)_{11} \times 11^{5}$ \\
\hline$p$ & $L_{p}\left(3, \chi_{\pi} \omega_{p}^{-2}, \mathbb{Q}\right)$ \\
\hline 2 & $(1.11110101101001001100111001001100001010010111011100011010101000010001)_{2} \times 2^{4}$ \\
\hline 3 & $(1.1221211120021010011021120022021022220202012220120020)_{3} \times 3^{0}$ \\
\hline 5 & $(1.03123432421241311210024333243134200)_{5} \times 5^{0}$ \\
\hline 7 & $(1.20515104501535164355503412253)_{7} \times 7^{0}$ \\
\hline 11 & $(4 . A 19905 A 97667183 A 61256312)_{11} \times 11^{0}$ \\
\hline$n=5$ & $e\left(5, M_{\pi}\right)^{-1}=2^{7} \cdot 3^{10} \cdot 5^{-2} \cdot 11^{7} \cdot 151 \cdot 1389251$ \\
\hline$p$ & $R_{5, p}\left(M_{\pi}\right) / D\left(M_{\pi}\right)^{1 / 2, p}$ \\
\hline 2 & $(1.10101000100011001001110000000110011010000011010001100100101100100011)_{2} \times 2^{16}$ \\
\hline 3 & $(2.00020011020011210121200021121000100102202022102012021120)_{3} \times 3^{10}$ \\
\hline 5 & $(3.043124340101142041302443131432222411101)_{5} \times 5^{8}$ \\
\hline 7 & $(3.1016042121151553442503432550426)_{7} \times 7^{10}$ \\
\hline 11 & $(4 . A 244 A A 713986547114383)_{11} \times 11^{13}$ \\
\hline$p$ & $L_{p}\left(5, \chi_{\pi} \omega_{p}^{-4}, \mathbb{Q}\right)$ \\
\hline 2 & $(1.01011001111000100100100110110111001110010101101110101100000001001101)_{2} \times 2^{4}$ \\
\hline 3 & $(1.2200111010220200001122120000000211211201112212022102)_{3} \times 3^{0}$ \\
\hline 5 & $(4.31111224412403210010123414333414223)_{5} \times 5^{0}$ \\
\hline 7 & $(2.63463651641424006321031014001)_{7} \times 7^{0}$ \\
\hline 11 & $(2.054143779893294 A 023 A 2760)_{11} \times 11^{1}$ \\
\hline
\end{tabular}

the discriminant are in Tables 5 through 8. The value that we found for (7.3) in this case was $1+O\left(p^{N(p)}\right)$ where $N(2) \geq 76, N(3) \geq 52, N(5) \geq 34, N(7) \geq 29$ and $N(11) \geq 22$. Such calculations were also carried out for the next four such extensions, with similar results.

Example 7.5. Let $k$ be a CM Galois extension of $\mathbb{Q}$ with $\operatorname{Gal}(k / \mathbb{Q}) \cong D_{8}$ as in Case 2 of Example 5.5, and use notation as in that example. We again write $M_{\pi}$ instead of $M_{\pi}^{E}$ since $E=\mathbb{Q}$, and consider even $n \geq 2$. Again $\pi K_{2 n-1}(k)_{\mathbb{Q}}=$ $\pi_{2} K_{2 n-1}\left(k_{1}\right)_{\mathbb{Q}}$, so we computed $K_{2 n-1}\left(k_{1}\right)_{\mathbb{Q}}$ numerically using the methods of Section 4 . We put our results at $n=2$ and 4 for the first four such extensions when ordered by the value of the (positive) discriminant in Tables 9 through 12 . The value that we found for (7.3) in this case was $1+O\left(p^{N(p)}\right)$ where $N(2) \geq 79$, $N(3) \geq 53, N(5) \geq 35, N(7) \geq 27$ and $N(11) \geq 25$. Such calculations were also carried out for the next four such extensions, with similar results. 
TABLE 7. Splitting field of $x^{4}-6 x^{2}+6$, where $C\left(\chi_{\pi}\right)=2^{7} \cdot 3^{2}$.

\begin{tabular}{|c|c|}
\hline$n=3$ & $e\left(3, M_{\pi}\right)^{-1}=-2^{8} \cdot 3^{2}$ \\
\hline$p$ & $R_{3, p}\left(M_{\pi}\right) / D\left(M_{\pi}\right)^{1 / 2, p}$ \\
\hline 2 & $(1.11111110110101110110001001001000100111010011011111000101011110110110)_{2} \times 2^{10}$ \\
\hline 3 & $(2.022112012020121000012010222021210101120000211220112222110100)_{3} \times 3^{2}$ \\
\hline 5 & $(2.041234240331110012313440303434111221114)_{5} \times 5^{7}$ \\
\hline 7 & $(4.126126412601443066506634321456330)_{7} \times 7^{6}$ \\
\hline 11 & $(1.508753 A 40028580 A 83265 A 6397)_{11} \times 11^{6}$ \\
\hline$p$ & $L_{p}\left(3, \chi_{\pi} \omega_{p}^{-2}, \mathbb{Q}\right)$ \\
\hline 2 & $(1.00111001111010100110000110000111111000001101110001010000100110100110)_{2} \times 2^{2}$ \\
\hline 3 & $(1.1102020120122110111012211100112220010211120222001022)_{3} \times 3^{0}$ \\
\hline 5 & $(3.24102423421030322330312322041041320)_{5} \times 5^{1}$ \\
\hline 7 & $(3.53421553111552403452502135351)_{7} \times 7^{0}$ \\
\hline 11 & $(9.993168 A 73395501045 A 926 A 6)_{11} \times 11^{0}$ \\
\hline$n=5$ & 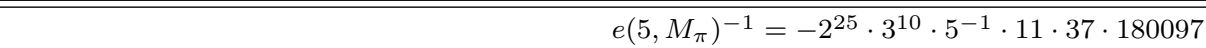 \\
\hline 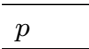 & $R_{5, p}\left(M_{\pi}\right) / D\left(M_{\pi}\right)^{1 / 2, p}$ \\
\hline 2 & $(1.01000011110101000111110110010101100000101100100001110011010100110111)_{2} \times 2^{27}$ \\
\hline 3 & $(1.20110210022121202120201021000120210110011111202222221202)_{3} \times 3^{10}$ \\
\hline 5 & $(4.13321010222213321143100244321412404344)_{5} \times 5^{9}$ \\
\hline 7 & $(1.6542260045104311315015635541014)_{7} \times 7^{10}$ \\
\hline 11 & $(4.35786968692892127976707)_{11} \times 11^{11}$ \\
\hline$p$ & $L_{p}\left(5, \chi_{\pi} \omega_{p}^{-4}, \mathbb{Q}\right)$ \\
\hline 2 & $(1.00100100101111111001101001011101011111110110100110100000111111010001)_{2} \times 2^{2}$ \\
\hline 3 & $(1.0101222010121001211100001210022220120122121200102122)_{3} \times 3^{0}$ \\
\hline 5 & $(2.44334240213144033340330341233100231)_{5} \times 5^{0}$ \\
\hline 7 & $(2.43613231343555620664002413300)_{7} \times 7^{0}$ \\
\hline 11 & $(A .957125591551731675713855) 11 \times 11^{0}$ \\
\hline
\end{tabular}

Example 7.6. Let $k$ be a totally real $S_{3} \times \mathbb{Z} / 3 \mathbb{Z}$ extension of $\mathbb{Q}$, as in Case 1 of Example 5.7. We use notation as in that example, taking $E=\mathbb{Q}\left(\zeta_{3}\right)$ for a primitive cube root of unity $\zeta_{3}$. A different identification of $\operatorname{Gal}(k / \mathbb{Q})$ with $S_{3} \times$ $\mathbb{Z} / 3 \mathbb{Z}$ might interchange $\pi_{3}$ and $\pi_{4}$ as well as corresponding primitive idempotents, but, as we noted before, the validity of the conjecture for either is equivalent.

Since $\pi K_{2 n-1}(k)_{E}=\pi_{3} K_{2 n-1}\left(s_{1}\right)_{E}$ we computed $K_{2 n-1}\left(s_{1}\right)_{\mathbb{Q}}$ as before. Also, in order to speed up the calculations of the regulator we note that the action of $(e, 1)$ and $\left(\sigma^{2}, 0\right)$ on $s_{1}=k^{\langle(\sigma, 1)\rangle}$ is the same, so that $\pi=\pi_{3} \pi_{H_{1}}$ and

$$
\left(1-\pi_{1}\right)\left((e, 0)+\zeta_{3}(\sigma, 0)+\zeta_{3}^{2}\left(\sigma^{2}, 0\right)\right) / 3=\left((e, 0)+\zeta_{3}(\sigma, 0)+\zeta_{3}^{2}\left(\sigma^{2}, 0\right)\right) / 3
$$

give the same result when applied to $K_{2 n-1}\left(s_{1}\right)_{E}$. We put our results for the first four $s_{1}$, when ordered according to their discriminant, for $n=3$ and 5 in 
TABLE 8. Splitting field of $x^{4}-6 x^{2}-4 x+2$, where $C\left(\chi_{\pi}\right)=2^{5} \cdot 17$.

\begin{tabular}{|c|c|}
\hline$n=3$ & $e\left(3, M_{\pi}\right)^{-1}=-2^{2} \cdot 3^{-2} \cdot 17^{2}$ \\
\hline$p$ & $R_{3, p}\left(M_{\pi}\right) / D\left(M_{\pi}\right)^{1 / 2, p}$ \\
\hline 2 & $(1.10111000110111011100110001011111110001011110100001100011100001101001)_{2} \times 2^{7}$ \\
\hline 3 & $(1.1001122110011102222210022111010102000101002122001210120111212)_{3} \times 3^{4}$ \\
\hline 5 & $(2.1330133431400143111041343243044132341000)_{5} \times 5^{6}$ \\
\hline 7 & $(1.251366021640656215554304534633666)_{7} \times 7^{6}$ \\
\hline 11 & $(6.8781153571182 A 7 A 3 A 52578590)_{11} \times 11^{6}$ \\
\hline$p$ & $L_{p}\left(3, \chi_{\pi} \omega_{p}^{-2}, \mathbb{Q}\right)$ \\
\hline 2 & $(1.01100101000011011110000111011100100110110110111011000100000101100110)_{2} \times 2^{5}$ \\
\hline 3 & $(2.2111111121010122120102220010120102202210101102021112)_{3} \times 3^{0}$ \\
\hline 5 & $(2.03010131243321342244201240202313243)_{5} \times 5^{0}$ \\
\hline 7 & $(2.45653546525345623002012601006)_{7} \times 7^{0}$ \\
\hline 11 & $(1.5054539309942 A 8686113521)_{11} \times 11^{0}$ \\
\hline$n=5$ & $e\left(5, M_{\pi}\right)^{-1}=-2^{14} \cdot 3^{2} \cdot 5^{-2} \cdot 17^{4}$ \\
\hline$p$ & $R_{5, p}\left(M_{\pi}\right) / D\left(M_{\pi}\right)^{1 / 2, p}$ \\
\hline 2 & $(1.01101001100111100011100000011101100011011111111011011010000000100111)_{2} \times 2^{19}$ \\
\hline 3 & $(1.0022021200111200200110022100110101220100021201020020021001)_{3} \times 3^{12}$ \\
\hline 5 & $(4.41122221322441142131444023001440141102)_{5} \times 5^{9}$ \\
\hline 7 & $(4.6425656621000232634542266362653)_{7} \times 7^{10}$ \\
\hline 11 & $(5.30669526 A 542413 A 15559106) 11 \times 11^{10}$ \\
\hline$p$ & $L_{p}\left(5, \chi_{\pi} \omega_{p}^{-4}, \mathbb{Q}\right)$ \\
\hline 2 & $(1.10000111001100100001010000010001000011100111101011000000110111100101)_{2} \times 2^{5}$ \\
\hline 3 & $(2.1100001122200222122120000002011201000121010120122102)_{3} \times 3^{0}$ \\
\hline 5 & $(1.00313434131411434403030110103232430)_{5} \times 5^{1}$ \\
\hline 7 & $(4.51404541504052312152404613255)_{7} \times 7^{0}$ \\
\hline 11 & $(2 . A 43315061300727 A 39739622)_{11} \times 11^{0}$ \\
\hline
\end{tabular}

Tables 13 through 16 . In Table 16 the number $e\left(5, M_{\pi}^{E}\right)^{-1}$ is given by

$$
-2^{5} \cdot 3^{10} \cdot 5^{-2} \cdot 13^{4} \cdot\left(7+3 \zeta_{3}\right) \cdot\left(68-43 \zeta_{3}\right) \cdot\left(1547202-1603487 \zeta_{3}\right) \cdot\left(a+b \zeta_{3}\right)
$$

with

$$
a=8482255892311139091186217686741233
$$

and

$$
b=3527440500058817421018094757947617,
$$

presumably because we experimentally found a subgroup of $K_{2 n-1}\left(s_{1}\right)_{\mathbb{Q}}$ that is not close to actually being $K_{2 n-1}\left(s_{1}\right)$. In all cases $L_{p}\left(n, \chi_{\pi} \otimes \omega_{p}^{1-n}, \mathbb{Q}\right)$ is a unit in $E \otimes_{\mathbb{Q}} \mathbb{Q}_{p}$, and when we write the equivalent of (7.3) in the form $1 \otimes \alpha+\zeta_{3} \otimes \beta$ then we find that $\alpha-1$ and $\beta$ are $O\left(p^{N(p)}\right)$ with $N(2) \geq 50, N(3) \geq 24, N(5) \geq 20$, 
TABLE 9. Splitting field of $x^{4}-2 x^{3}+5 x^{2}-4 x+2$, where $C\left(\chi_{\pi}\right)=2^{3} \cdot 17$.

\begin{tabular}{|c|c|}
\hline$n=2$ & $e\left(2, M_{\pi}\right)^{-1}=-17$ \\
\hline$p$ & $R_{2, p}\left(M_{\pi}\right) / D\left(M_{\pi}\right)^{1 / 2, p}$ \\
\hline 2 & $(1.11110000011111000100011010100111010001000101111111000010111001000001)_{2} \times 2^{8}$ \\
\hline 3 & $(1.2001010102220112101111101220000000012020211022222012112010122)_{3} \times 3^{4}$ \\
\hline 5 & $(2.01203020434342403202140220421203111323421)_{5} \times 5^{4}$ \\
\hline 7 & $(4.1140305454665411023106103225364300)_{7} \times 7^{4}$ \\
\hline 11 & $(9.074667649 A 54466861880828 A 41)_{11} \times 11^{4}$ \\
\hline$p$ & $L_{p}\left(2, \chi_{\pi} \omega_{p}^{-1}, \mathbb{Q}\right)$ \\
\hline 2 & $(1.10011100010001001110011010011101110110111100101101100011101101010011)_{2} \times 2^{6}$ \\
\hline 3 & $(1.2211021011200210000101010021221011201210202220121211)_{3} \times 3^{0}$ \\
\hline 5 & $(4.33003403101112402103034043124123020)_{5} \times 5^{0}$ \\
\hline 7 & $(6.55655254400302602216266140200)_{7} \times 7^{0}$ \\
\hline 11 & $(4.84968 A A A 0466783629843316)_{11} \times 11^{0}$ \\
\hline$n=4$ & $\overline{e\left(4, M_{\pi}\right)^{-1}=2^{4} \cdot 3^{2} \cdot 17^{3}}$ \\
\hline$p$ & $R_{4, p}\left(M_{\pi}\right) / D\left(M_{\pi}\right)^{1 / 2, p}$ \\
\hline 2 & $(1.01111010101011110100100110010011000001110001101011101000010011010010)_{2} \times 2^{15}$ \\
\hline 3 & $(2.02020202222222212011121000222200020002221002201220201002211)_{3} \times 3^{10}$ \\
\hline 5 & $(3.121141434441031100040041101320123332342)_{5} \times 5^{8}$ \\
\hline 7 & $(4.30103246365105165401451263010636)_{7} \times 7^{8}$ \\
\hline 11 & $(9.8864321414815928 A 83132426)_{11} \times 11^{8}$ \\
\hline$p$ & $L_{p}\left(4, \chi_{\pi} \omega_{p}^{-3}, \mathbb{Q}\right)$ \\
\hline 2 & $(1.10101000111010101100110000110001001011001001001001010011001010011101)_{2} \times 2^{7}$ \\
\hline 3 & $(1.0220110000101201002201220101112220112012211122211111)_{3} \times 3^{0}$ \\
\hline 5 & $(4.24124003322330023340322023204112410)_{5} \times 5^{0}$ \\
\hline 7 & $(1.34631136124161206563156313232)_{7} \times 7^{0}$ \\
\hline 11 & $(6.484018160860423737043861)_{11} \times 11^{0}$ \\
\hline
\end{tabular}

$N(7) \geq 14$ and $N(11) \geq 12$, with in this case the precision bounded by that of the $R_{n, p}\left(M_{\pi}^{E}\right)$. Since $e\left(n, M_{\pi}^{E}\right), D\left(M_{\pi}^{E}\right)^{1 / 2, p}$ and $\operatorname{Eul}_{p}\left(n, \chi_{\pi} \otimes \mathrm{id}, \mathbb{Q}\right)$ are units in $E \otimes_{\mathbb{Q}} F$ this also shows that $R_{n, p}\left(M_{\pi}^{E}\right.$ ) is a unit in $E \otimes_{\mathbb{Q}} F$ (as conjectured in Conjecture 3.18(4)) for $n=3$ and makes it very plausible for $n=5$.

Such calculations were also carried out for the next four such $s_{1}$ with similar results.

Remark 7.7. In Example 7.1, the conjecture for $\pi$ and the non-vanishing of $L_{p}\left(n, \omega_{p}^{1-n}, \mathbb{Q}\right)$ are equivalent to Conjecture 2.17 for the field $c$ in the example by Proposition 4.17 and Remark 3.21(1). Also, part (1) of the conjecture holds for $\pi$ by Proposition 5.9. The same statements are true for $\pi$ and $k_{1}$ in Example 7.4.

Remark 7.8. In the tables we have also included the value of the constant $C\left(\chi_{\pi} \otimes \mathrm{id}\right)=C\left(\chi_{\pi}^{\vee} \otimes \mathrm{id}\right)$ in the functional equation $(3.25)$ of $L\left(s, \chi_{\pi} \otimes \mathrm{id}, \mathbb{Q}\right)$ 
TABLE 10. Splitting field of $x^{4}-x^{3}+3 x^{2}-2 x+4$, where $C\left(\chi_{\pi}\right)=5 \cdot 41$.

\begin{tabular}{|c|c|}
\hline$n=2$ & $e\left(2, M_{\pi}\right)^{-1}=2^{-3} \cdot 5 \cdot 41$ \\
\hline$p$ & $R_{2, p}\left(M_{\pi}\right) / D\left(M_{\pi}\right)^{1 / 2, p}$ \\
\hline 2 & $(1.00100101101010001111110110001011010111110011100101011001110011011000)_{2} \times 2^{6}$ \\
\hline 3 & $(2.0021220111220210202122211101102111121010022202210012111112202)_{3} \times 3^{4}$ \\
\hline 5 & $(4.2413230212134320403404223440242144143010)_{5} \times 5^{4}$ \\
\hline 7 & $(5.6622665100264660251241453165220553)_{7} \times 7^{4}$ \\
\hline 11 & $(2.0 A 380682536 A 50856633 A 5963 A A)_{11} \times 11^{4}$ \\
\hline$p$ & $L_{p}\left(2, \chi_{\pi} \omega_{p}^{-1}, \mathbb{Q}\right)$ \\
\hline 2 & $(1.10001100100010010110110100011001000110001101100111111011111110000000)_{2} \times 2^{5}$ \\
\hline 3 & $(1.0000112110000221101100101100220112122121100200111220)_{3} \times 3^{0}$ \\
\hline 5 & $(3.31322324200110323444242424141204000)_{5} \times 5^{1}$ \\
\hline 7 & $(6.20642211231410105634103112315)_{7} \times 7^{0}$ \\
\hline 11 & $(4.8488929 A A 248277415458863)_{11} \times 11^{0}$ \\
\hline$n=4$ & $e\left(4, M_{\pi}\right)^{-1}=2^{-5} \cdot 3^{2} \cdot 5^{3} \cdot 41^{3}$ \\
\hline$p$ & $R_{4, p}\left(M_{\pi}\right) / D\left(M_{\pi}\right)^{1 / 2, p}$ \\
\hline 2 & $(1.01101011011011001001110001011011001001100001000100001111001011100010)_{2} \times 2^{9}$ \\
\hline 3 & $(2.12201221002111122111110002102122122100002111212000210102220)_{3} \times 3^{10}$ \\
\hline 5 & $(3.03433213241214232102131324212010224400)_{5} \times 5^{8}$ \\
\hline 7 & $(3.21341424531605465104510422214051)_{7} \times 7^{8}$ \\
\hline 11 & $(A .180567 A A 0545 A 59652909 A 250)_{11} \times 11^{8}$ \\
\hline$p$ & $L_{p}\left(4, \chi_{\pi} \omega_{p}^{-3}, \mathbb{Q}\right)$ \\
\hline 2 & $(1.11100001111100101110011000101000001100001100100001000011000001000111)_{2} \times 2^{6}$ \\
\hline 3 & $(1.2110210100202111000001112120121020120200000202111102)_{3} \times 3^{0}$ \\
\hline 5 & $(1.22414201312142130131112334430123200)_{5} \times 5^{1}$ \\
\hline 7 & $(6.66200605216643655434600606423)_{7} \times 7^{0}$ \\
\hline 11 & $(3.5059939560 A 2 A A 91106844 A 7)_{11} \times 11^{0}$ \\
\hline
\end{tabular}

to make possible a comparison between its prime factors and those of $e\left(n, M_{\pi}\right)$. Namely, for the $D\left(M_{\pi}^{E}\right)^{1 / 2, \infty}$ used in the calculations, the $e_{n}$ in (3.26) (which in our case are independent of $n$ ) are as follows.

\begin{tabular}{cccccccc}
\hline Table & $e_{n}$ & Table & $e_{n}$ & Table & $e_{n}$ & Table & $e_{n}$ \\
\hline 1 & 3 & 5 & 4 & 9 & 4 & 13 & $1 \otimes 9+\zeta_{3} \otimes 9$ \\
2 & 3 & 6 & 4 & 10 & 4 & 14 & $1 \otimes 9+\zeta_{3} \otimes 9$ \\
3 & 3 & 7 & 4 & 11 & -4 & 15 & $1 \otimes 0+\zeta_{3} \otimes 9$ \\
4 & 3 & 8 & 4 & 12 & 4 & 16 & $1 \otimes 0+\zeta_{3} \otimes(-9)$ \\
\hline
\end{tabular}

As can be seen from these values and Tables 1-16, in the number

$$
\delta_{n} e_{n}((n-1) ! / 2)^{m} C\left(\chi_{\pi}^{\vee} \otimes \mathrm{id}\right)^{n-1} e\left(n, M_{\pi}^{E}\right)
$$


TABLE 11. Splitting field of $x^{4}-2 x^{3}+6 x^{2}-5 x+2$, where $C\left(\chi_{\pi}\right)=13 \cdot 17$.

\begin{tabular}{|c|c|}
\hline$n=2$ & $e\left(2, M_{\pi}\right)^{-1}=2^{-3} \cdot 13 \cdot 17$ \\
\hline$p$ & $R_{2, p}\left(M_{\pi}\right) / D\left(M_{\pi}\right)^{1 / 2, p}$ \\
\hline 2 & $(1.11100111011011110110000110110011010010001100010000011011000000100011)_{2} \times 2^{7}$ \\
\hline 3 & $(1.001110110101022221010202101001220021110111221112100200010011)_{3} \times 3^{5}$ \\
\hline 5 & $(4.12002342224442011223100142021013114003331)_{5} \times 5^{4}$ \\
\hline 7 & $(6.0065051533250322312551556340446112)_{7} \times 7^{4}$ \\
\hline 11 & $(9.58602382412 A 681650341722097)_{11} \times 11^{4}$ \\
\hline$p$ & $L_{p}\left(2, \chi_{\pi} \omega_{p}^{-1}, \mathbb{Q}\right)$ \\
\hline 2 & $(1.01010001000000000001000000101011111111001100001000001100111111100100)_{2} \times 2^{6}$ \\
\hline 3 & $(2.0212122200002021202200211122100200001001012201202000)_{3} \times 3^{1}$ \\
\hline 5 & $(2.12300004023110442141103112001044200)_{5} \times 5^{0}$ \\
\hline 7 & $(5.65035644055466446205106662353)_{7} \times 7^{0}$ \\
\hline 11 & $(6.38803 A 213738 A 731776470 A 4)_{11} \times 11^{0}$ \\
\hline$n=4$ & $e\left(4, M_{\pi}\right)^{-1}=-2^{-5} \cdot 3^{2} \cdot 13^{3} \cdot 17^{3}$ \\
\hline$p$ & $R_{4, p}\left(M_{\pi}\right) / D\left(M_{\pi}\right)^{1 / 2, p}$ \\
\hline 2 & $(1.01010110010100100110100011110011010001011010001010001001110111101010)_{2} \times 2^{10}$ \\
\hline 3 & $(1.1111010110110112222112002001211122102011200022210022220110)_{3} \times 3^{11}$ \\
\hline 5 & $(2.300041000440134413002331411231121430312)_{5} \times 5^{8}$ \\
\hline 7 & $(2.63246241360463115315231424221502)_{7} \times 7^{8}$ \\
\hline 11 & $(A .49299953329 A 1125214 A 939 A 7)_{11} \times 11^{8}$ \\
\hline$p$ & $L_{p}\left(4, \chi_{\pi} \omega_{p}^{-3}, \mathbb{Q}\right)$ \\
\hline 2 & $(1.00100111010100001110011000011111110110110001001100110010111000011110)_{2} \times 2^{7}$ \\
\hline 3 & $(1.2001110201210120022112022021221001220010010202222220)_{3} \times 3^{1}$ \\
\hline 5 & $(4.31111034231223303224440300102101300)_{5} \times 5^{0}$ \\
\hline 7 & $(3.23250645010664211233114156026)_{7} \times 7^{0}$ \\
\hline 11 & $(6.722 A 82921527466612856704)_{11} \times 11^{0}$ \\
\hline
\end{tabular}

of (3.27) the larger prime factors occurring in $e\left(n, M_{\pi}^{E}\right)$ are often cancelled by those of $C\left(\chi_{\pi}^{\vee} \otimes \mathrm{id}\right)^{n-1}$. This suggests that the formulation of Beilinson's conjecture at $s=1-n$ normally involves simpler prime factors in $E^{*}$ than the formulation at $s=n$ in Conjecture 3.18(1), with the remaining complicated factors in our examples quite possibly due to an awkward choice of basis for $K_{2 n-1}\left(M_{\pi}^{E}\right)$.

\section{REFERENCES}

[1] D. Barsky. Fonctions zêta $p$-adiques d'une classe de rayon des corps de nombres totalement réels. In Groupe d'Etude d'Analyse Ultramétrique (5e année: 197r/78), pages Exp. No. 16, 23. Secrétariat Math., Paris, 1978.

[2] A. A. Beilinson. Higher regulators and values of $L$-functions. J. Sov. Math., 30:2036-2070, 1985.

[3] A. Besser. Syntomic regulators and p-adic integration I: rigid syntomic regulators. Israel Journal of Math., 120:291-334, 2000. 
TABLE 12. Splitting field of $x^{4}+3 x^{2}-6 x+6$, where $C\left(\chi_{\pi}\right)=2^{2} \cdot 3^{2} \cdot 11$.

\begin{tabular}{|c|c|}
\hline$n=2$ & $e\left(2, M_{\pi}\right)^{-1}=-2^{-1} \cdot 3^{2} \cdot 11$ \\
\hline$p$ & $R_{2, p}\left(M_{\pi}\right) / D\left(M_{\pi}\right)^{1 / 2, p}$ \\
\hline 2 & $(1.00011111110011010111101100110001100000010001000010010011001000110100)_{2} \times 2^{6}$ \\
\hline 3 & $(2.001121000222211121002102201101101100211202000001120001201101)_{3} \times 3^{2}$ \\
\hline 5 & $(4.32211042044110431112434230011130322040334)_{5} \times 5^{4}$ \\
\hline 7 & $(6.460003406606016165433631101363300)_{7} \times 7^{5}$ \\
\hline 11 & $(4.355421 A 54525023347874 A 49229)_{11} \times 11^{3}$ \\
\hline$p$ & $L_{p}\left(2, \chi_{\pi} \omega_{p}^{-1}, \mathbb{Q}\right)$ \\
\hline 2 & $(1.11101000100101000011100111111110011101011000001101111010111100101100)_{2} \times 2^{5}$ \\
\hline 3 & $(1.2022010222110011100120101011011011020010020011221012)_{3} \times 3^{0}$ \\
\hline 5 & $(3.22012020201133014223242323022213400)_{5} \times 5^{0}$ \\
\hline 7 & $(2.43314511556206561022451602200)_{7} \times 7^{1}$ \\
\hline 11 & $(7.514814537963976728 A 46001) 11 \times 11^{0}$ \\
\hline$n=4$ & $e\left(4, M_{\pi}\right)^{-1}=-2 \cdot 3^{8} \cdot 11^{3}$ \\
\hline$p$ & $R_{4, p}\left(M_{\pi}\right) / D\left(M_{\pi}\right)^{1 / 2, p}$ \\
\hline 2 & $(1.00011101001000000111110111000101001111110011011101001101001001110101)_{2} \times 2^{11}$ \\
\hline 3 & $(2.0222010120001012000101112011222120000210000112021000101001)_{3} \times 3^{8}$ \\
\hline 5 & $(4.200024100322411004323111441041141020122)_{5} \times 5^{8}$ \\
\hline 7 & $(5.12536440256103300364331512021150)_{7} \times 7^{8}$ \\
\hline 11 & $(6.6966231827671 A 91984 A 434 A 7)_{11} \times 11^{7}$ \\
\hline$p$ & $L_{p}\left(4, \chi_{\pi} \omega_{p}^{-3}, \mathbb{Q}\right)$ \\
\hline 2 & $(1.10111000101101010100110110100100101011000111100000010010100110001101)_{2} \times 2^{6}$ \\
\hline 3 & $(1.0221110122220100011212020012222021100000010022110011)_{3} \times 3^{0}$ \\
\hline 5 & $(3.44200104443132341021214030231112200)_{5} \times 5^{0}$ \\
\hline 7 & $(4.44003540500226200364534021346)_{7} \times 7^{0}$ \\
\hline 11 & $(5.766584290652809 A 28501199)_{11} \times 11^{0}$ \\
\hline
\end{tabular}

[4] A. Besser. Syntomic regulators and p-adic integration II: $K_{2}$ of curves. Israel Journal of Math., 120:335-360, 2000.

[5] A. Besser and R. de Jeu. The syntomic regulator for the $K$-theory of fields. Annales Scientifiques de l'École Normale Supérieure, 36(6):867-924, 2003.

[6] A. Besser and R. de Jeu. $L i^{(p)}$-service? An algorithm for computing $p$-adic polylogarithms. Math. Comp., 77:1105-1134, 2008.

[7] A. Besser and C. Deninger. p-adic Mahler measures. J. Reine Angew. Math., 517:19-50, 1999.

[8] F. Beukers. Irrationality of some p-adic L-values. Acta Math. Sin. (Engl. Ser.), 24:663-686, 2008.

[9] S. Bloch. Higher regulators, algebraic $K$-theory, and zeta functions of elliptic curves. Manuscript from 1978 ("Irvine notes"). Published as volume 11 of CRM Monographs Series by American Mathematical Society.

[10] A. Borel. Stable real cohomology of arithmetic groups. Ann. Sci. ENS, 4:235-272, 1974. 
TABLE 13. Splitting field of $x^{6}-x^{5}-6 x^{4}+7 x^{3}+4 x^{2}-5 x+1$, where $C\left(\chi_{\pi} \otimes \mathrm{id}\right)=2^{2} \cdot 5 \cdot 19$.

\begin{tabular}{lc}
\hline$n=3$ & $e\left(3, M_{\pi}^{E}\right)^{-1}=3^{-2} \cdot 19^{2}$ \\
\hline$p$ & $1 \otimes\left((1.010101011100101010)_{2} \times 2^{4}\right)+\zeta_{3} \otimes\left((1.111100111000011101)_{2} \times 2^{2}\right)$ \\
\hline 2 & $1 \otimes\left((2.121122121210110121)_{3} \times 3^{4}\right)+\zeta_{3} \otimes\left((1.100202121120121202)_{3} \times 3^{4}\right)$ \\
3 & $1 \otimes\left((4.402020001241122012)_{5} \times 5^{3}\right)+\zeta_{3} \otimes\left((1.402102204113114001)_{5} \times 5^{4}\right)$ \\
5 & $1 \otimes\left((2.1404154326422162)_{7} \times 7^{6}\right)+\zeta_{3} \otimes\left((5.1444362630564332)_{7} \times 7^{6}\right)$ \\
7 & $1 \otimes\left((6.9026829596822)_{11} \times 11^{6}\right)+\zeta_{3} \otimes\left((8.048680429402 A)_{11} \times 11^{6}\right)$ \\
\hline 1 & $L_{p}\left(3, \chi_{\pi} \otimes \omega_{p}^{-2}, \mathbb{Q}\right)$ \\
\hline 2 & $1 \otimes\left((1.010111010101101000)_{2} \times 2^{4}\right)+\zeta_{3} \otimes\left((1.111111110001001011)_{2} \times 2^{2}\right)$ \\
3 & $1 \otimes\left((1.120012101022112222)_{3} \times 3^{0}\right)+\zeta_{3} \otimes\left((2.212202101110022110)_{3} \times 3^{0}\right)$ \\
5 & $1 \otimes\left((6.215644553551135401)_{7} \times 7^{0}\right)+\zeta_{3} \otimes\left((1.642236344041330514)_{7} \times 7^{0}\right)$ \\
7 & $1 \otimes\left((6.6 A 0036761338492361)_{11} \times 11^{0}\right)+\zeta_{3} \otimes\left((8.749011 A A 3694540145)_{11} \times 11^{0}\right)$ \\
11 & $\left.R_{5, p}\left(M_{\pi}^{E}\right) / D\left(M_{\pi}\right)^{1}\right)^{1 / 2, p}$ \\
\hline \hline$n=5$ & $1 \otimes\left((1.110110120010021222)_{3} \times 3^{12}\right)+\zeta_{3} \otimes\left((2.020220021122220210)_{3} \times 3^{12}\right)$ \\
\hline$p$ & $1 \otimes\left((3.020013014242432200)_{5} \times 5^{7}\right)+\zeta_{3} \otimes\left((1.220134043203242432)_{5} \times 5^{7}\right)$ \\
\hline 2 & $1 \otimes\left((4.15141551064450)_{7} \times 7^{10}\right)+\zeta_{3} \otimes\left((6.53120060244502)_{7} \times 7^{10}\right)$ \\
3 & $1 \otimes\left((4.285500134)_{11} \times 11^{12}\right)+\zeta_{3} \otimes\left((2 . A 3934821906)_{11} \times 11^{10}\right)$ \\
5 & $L_{p}\left(5, \chi_{\pi} \otimes \omega_{p}^{-4}, \mathbb{Q}\right)$ \\
7 & $1 \otimes\left(\left((1.110010010001000101)_{2} \times 2^{4}\right)+\zeta_{3} \otimes\left((1.111111100101010110)_{2} \times 2^{2}\right)\right.$ \\
11 & $1 \otimes\left((1.211021010101222221)_{3} \times 3^{0}\right)+\zeta_{3} \otimes\left((2.122212200211101011)_{3} \times 3^{0}\right)$ \\
\hline$p$ & $\left.(6.3 A 4 A 10 A 60052302518)_{11} \times 11^{0}\right)+\zeta_{3} \otimes\left((4.7 A A 8843572652457 A 4)_{11} \times 11^{0}\right)$ \\
\hline 2 & $\left.1.401222401123304240)_{5} \times 5^{0}\right)+\zeta_{3} \otimes\left((1.432224122043203014)_{5} \times 5^{0}\right)$ \\
3 & $\left.1.441442356342000541)_{7} \times 7^{0}\right)+\zeta_{3} \otimes\left((3.664531413660053644)_{7} \times 7^{0}\right)$ \\
\hline 7 & $\left.1.000011100001001101)_{2} \times 2^{9}\right)+\zeta_{3} \otimes\left((1.101010111001110110)_{2} \times 2^{9}\right)$ \\
\hline
\end{tabular}

[11] A. Borel. Cohomologie de $\mathrm{SL}_{n}$ et valeurs de fonctions zêta aux points entiers. Ann. Scuola Norm. Sup. Pisa Cl. Sci. (4), 4(4):613-636, 1977. Errata at vol. 7, p. 373 (1980).

[12] J. I. Burgos Gil. The regulators of Beilinson and Borel, volume 15 of CRM Monograph Series. Amer. Math. Soc., Providence, RI, 2002.

[13] F. Calegari. Irrationality of certain p-adic periods for small p. Int. Math. Res. Not., (20):1235-1249, 2005.

[14] P. Cassou-Noguès. $p$-adic $L$-functions for totally real number field. In Proceedings of the Conference on p-adic Analysis (Nijmegen, 1978), volume 7806 of Report, pages 24-37. Katholieke Univ. Nijmegen, 1978.

[15] P. Cassou-Noguès. Valeurs aux entiers négatifs des fonctions zêta et fonctions zêta $p$-adiques. Invent. Math., 51(1):29-59, 1979.

[16] R. Coleman. Dilogarithms, regulators, and p-adic L-functions. Invent. Math., 69:171-208, 1982.

[17] R. Coleman and B. Gross. p-adic heights on curves. In Algebraic number theory, pages 73-81. Academic Press, Boston, MA, 1989. 
TABLE 14. Splitting field of $x^{6}-x^{5}-8 x^{4}-x^{3}+12 x^{2}+7 x+1$, where $C\left(\chi_{\pi} \otimes\right.$ id $)=2^{2} \cdot 5 \cdot 31$.

\begin{tabular}{|c|c|}
\hline$n=3$ & $e\left(3, M_{\pi}^{E}\right)^{-1}=2^{-2} \cdot 3^{-2} \cdot 31^{2} \cdot \zeta_{3}$ \\
\hline$p$ & $R_{3, p}\left(M_{\pi}^{E}\right) / D\left(M_{\pi}^{E}\right)^{1 / 2, p}$ \\
\hline 2 & $1 \otimes\left((1.100100010111101000)_{2} \times 2^{2}\right)+\zeta_{3} \otimes\left((1.111010100101111010)_{2} \times 2^{0}\right)$ \\
\hline 3 & $1 \otimes\left((1.221220200011201020)_{3} \times 3^{4}\right)+\zeta_{3} \otimes\left((2.020111011200022220)_{3} \times 3^{4}\right)$ \\
\hline 5 & $1 \otimes\left((1.141130342002111122)_{5} \times 5^{3}\right)+\zeta_{3} \otimes\left((4.342032011212400441)_{5} \times 5^{3}\right)$ \\
\hline 7 & $1 \otimes\left((4.4505556355566640)_{7} \times 7^{6}\right)+\zeta_{3} \otimes\left((3.0610023664215014)_{7} \times 7^{6}\right)$ \\
\hline 11 & $1 \otimes\left((7.3700473650 A 19)_{11} \times 11^{6}\right)+\zeta_{3} \otimes\left((1.3659 A 52396880)_{11} \times 11^{6}\right)$ \\
\hline$p$ & $L_{p}\left(3, \chi_{\pi} \otimes \omega_{p}^{-2}, \mathbb{Q}\right)$ \\
\hline 2 & $1 \otimes\left((1.101110001100101000)_{2} \times 2^{2}\right)+\zeta_{3} \otimes\left((1.010100111010111101)_{2} \times 2^{4}\right)$ \\
\hline 3 & $1 \otimes\left((2.111112222201000112)_{3} \times 3^{0}\right)+\zeta_{3} \otimes\left((1.120112022212020122)_{3} \times 3^{0}\right)$ \\
\hline 5 & $1 \otimes\left((4.321142113321234330)_{5} \times 5^{0}\right)+\zeta_{3} \otimes\left((1.130134403242024422)_{5} \times 5^{0}\right)$ \\
\hline 7 & $1 \otimes\left((5.530006300632330553)_{7} \times 7^{0}\right)+\zeta_{3} \otimes\left((2.200601212403230506)_{7} \times 7^{0}\right)$ \\
\hline 11 & $1 \otimes\left((2.00762927347972431 A)_{11} \times 11^{0}\right)+\zeta_{3} \otimes\left((A .191 A 1780051997 A 650)_{11} \times 11^{0}\right)$ \\
\hline$n=5$ & $e\left(5, M_{\pi}^{E}\right)^{-1}=-2^{6} \cdot 3^{3} \cdot 5^{2} \cdot 31^{4} \cdot\left(2+\zeta_{3}\right) \cdot\left(2-\zeta_{3}\right) \cdot\left(7-15 \zeta_{3}\right) \cdot\left(54+31 \zeta_{3}\right)$ \\
\hline$p$ & $R_{5, p}\left(M_{\pi}^{E}\right) / D\left(M_{\pi}^{E}\right)^{1 / 2, p}$ \\
\hline 2 & $1 \otimes\left((1.011100011000010010)_{2} \times 2^{8}\right)+\zeta_{3} \otimes\left((1.011100000000101011)_{2} \times 2^{8}\right)$ \\
\hline 3 & $1 \otimes\left((2.202202022111202222)_{3} \times 3^{14}\right)+\zeta_{3} \otimes\left((1.112002110200111001)_{3} \times 3^{15}\right)$ \\
\hline 5 & $1 \otimes\left((3.141433024141141322)_{5} \times 5^{8}\right)+\zeta_{3} \otimes\left((2.002014030014311244)_{5} \times 5^{7}\right)$ \\
\hline 7 & $1 \otimes\left((6.50135302661002)_{7} \times 7^{10}\right)+\zeta_{3} \otimes\left((4.04356114536113)_{7} \times 7^{10}\right)$ \\
\hline 11 & $1 \otimes\left((5 . A 3306 A 68921)_{11} \times 11^{10}\right)+\zeta_{3} \otimes\left((3.85 A 6650344 A)_{11} \times 11^{10}\right)$ \\
\hline$p$ & $L_{p}\left(5, \chi_{\pi} \otimes \omega_{p}^{-4}, \mathbb{Q}\right)$ \\
\hline 2 & $1 \otimes\left((1.100110110011011000)_{2} \times 2^{2}\right)+\zeta_{3} \otimes\left((1.110110100001011101)_{2} \times 2^{4}\right)$ \\
\hline 3 & $1 \otimes\left((2.201101211210020202)_{3} \times 3^{0}\right)+\zeta_{3} \otimes\left((1.212021202011010201)_{3} \times 3^{0}\right)$ \\
\hline 5 & $1 \otimes\left((4.011013012410044000)_{5} \times 5^{0}\right)+\zeta_{3} \otimes\left((1.431333301302323324)_{5} \times 5^{0}\right)$ \\
\hline 7 & $1 \otimes\left((2.130552365243450401)_{7} \times 7^{0}\right)+\zeta_{3} \otimes\left((2.601221155363314062)_{7} \times 7^{0}\right)$ \\
\hline 11 & $1 \otimes\left((9.53274 A 487 A 14818064)_{11} \times 11^{0}\right)+\zeta_{3} \otimes\left((9.89 A 625650825167861)_{11} \times 11^{0}\right)$ \\
\hline
\end{tabular}

[18] P. Colmez. Résidu en $s=1$ des fonctions zêta $p$-adiques. Invent. Math., 91(2):371-389, 1988.

[19] R. de Jeu. Zagier's conjecture and wedge complexes in algebraic K-theory. Compositio Mathematica, 96:197-247, 1995.

[20] P. Deligne. Valeurs de fonctions $L$ et périodes d'intégrales. In Automorphic forms, representations and L-functions (Proc. Sympos. Pure Math., Oregon State Univ., Corvallis, Ore., 1977), Part 2, Proc. Sympos. Pure Math., XXXIII, pages 313-346. Amer. Math. Soc., Providence, R.I., 1979. With an appendix by N. Koblitz and A. Ogus.

[21] P. Deligne. Interprétation motivique de la conjecture de Zagier reliant polylogarithms et régulateurs. Preprint, 1991.

[22] P. Deligne and K. Ribet. Values of abelian $L$-functions at negative integers over totally real fields. Invent. Math., 59(3):227-286, 1980.

[23] C. Deninger and A. J. Scholl. The Beĭlinson conjectures. In L-functions and arithmetic (Durham, 1989), volume 153 of London Math. Soc. Lecture Note Ser., pages 173-209. Cambridge Univ. Press, Cambridge, 1991. 
TABLE 15 . Splitting field of $x^{6}-x^{5}-8 x^{4}+5 x^{3}+19 x^{2}-4 x-11$, where $C\left(\chi_{\pi} \otimes\right.$ id $)=5 \cdot 139$.

\begin{tabular}{|c|c|}
\hline$n=3$ & $e\left(3, M_{\pi}^{E}\right)^{-1}=2^{-5} \cdot 3^{-2} \cdot 139^{2}$ \\
\hline$p$ & $R_{3, p}\left(M_{\pi}^{E}\right) / D\left(M_{\pi}^{E}\right)^{1 / 2, p}$ \\
\hline 2 & $1 \otimes\left((1.000110100111100100)_{2} \times 2^{4}\right)+\zeta_{3} \otimes\left((1.000011011101110010)_{2} \times 2^{5}\right)$ \\
\hline 3 & $1 \otimes\left((2.102212010212021001)_{3} \times 3^{4}\right)+\zeta_{3} \otimes\left((2.122111112211000220)_{3} \times 3^{4}\right)$ \\
\hline 5 & $1 \otimes\left((1.400211310114320013)_{5} \times 5^{3}\right)+\zeta_{3} \otimes\left((1.434230430334022423)_{5} \times 5^{3}\right)$ \\
\hline 7 & $1 \otimes\left((2.2523154003106061)_{7} \times 7^{6}\right)+\zeta_{3} \otimes\left((2.3200021260361110)_{7} \times 7^{6}\right)$ \\
\hline 11 & $1 \otimes\left((9.4472801 A 61 A 44)_{11} \times 11^{6}\right)+\zeta_{3} \otimes\left((1.7232221727046)_{11} \times 11^{6}\right)$ \\
\hline$p$ & $L_{p}\left(3, \chi_{\pi} \otimes \omega_{p}^{-2}, \mathbb{Q}\right)$ \\
\hline 2 & $1 \otimes\left((1.000100101010000101)_{2} \times 2^{4}\right)+\zeta_{3} \otimes\left((1.000010100000000110)_{2} \times 2^{3}\right)$ \\
\hline 3 & $1 \otimes\left((2.111012101221111220)_{3} \times 3^{2}\right)+\zeta_{3} \otimes\left((1.010011111201220212)_{3} \times 3^{0}\right)$ \\
\hline 5 & $1 \otimes\left((3.240243311024013404)_{5} \times 5^{0}\right)+\zeta_{3} \otimes\left((4.142342004244233331)_{5} \times 5^{2}\right)$ \\
\hline 7 & $1 \otimes\left((6.543343430631523116)_{7} \times 7^{1}\right)+\zeta_{3} \otimes\left((2.342260265363120032)_{7} \times 7^{0}\right)$ \\
\hline 11 & $1 \otimes\left((4.8521241 A 647 A 883282)_{11} \times 11^{0}\right)+\zeta_{3} \otimes\left((1.46220220356163464 A)_{11} \times 11^{0}\right)$ \\
\hline$n=5$ & $e\left(5, M_{\pi}^{E}\right)^{-1}=2^{-4} \cdot 3^{2} \cdot 5^{2} \cdot 139^{4} \cdot\left(4-5 \zeta_{3}\right)$ \\
\hline$p$ & $R_{5, p}\left(M_{\pi}^{E}\right) / D\left(M_{\pi}^{E}\right)^{1 / 2, p}$ \\
\hline 2 & $1 \otimes\left((1.000100111100110101)_{2} \times 2^{10}\right)+\zeta_{3} \otimes\left((1.011111001011110010)_{2} \times 2^{9}\right)$ \\
\hline 3 & $1 \otimes\left((1.202001002112012221)_{3} \times 3^{13}\right)+\zeta_{3} \otimes\left((1.021221211222021020)_{3} \times 3^{12}\right)$ \\
\hline 5 & $1 \otimes\left((3.404332041431011004)_{5} \times 5^{7}\right)+\zeta_{3} \otimes\left((3.213210304101144004)_{5} \times 5^{7}\right)$ \\
\hline 7 & $1 \otimes\left((4.11031052300040)_{7} \times 7^{10}\right)+\zeta_{3} \otimes\left((4.03411453000015)_{7} \times 7^{10}\right)$ \\
\hline 11 & $1 \otimes\left((6.8523188534)_{11} \times 11^{11}\right)+\zeta_{3} \otimes\left((5.2 A 39 A 447198)_{11} \times 11^{10}\right)$ \\
\hline$p$ & $L_{p}\left(5, \chi_{\pi} \otimes \omega_{p}^{-4}, \mathbb{Q}\right)$ \\
\hline 2 & $1 \otimes\left((1.101001011001000000)_{2} \times 2^{4}\right)+\zeta_{3} \otimes\left((1.000100011110101010)_{2} \times 2^{3}\right)$ \\
\hline 3 & $1 \otimes\left((1.200212010102010102)_{3} \times 3^{1}\right)+\zeta_{3} \otimes\left((1.122011112111021002)_{3} \times 3^{0}\right)$ \\
\hline 5 & $1 \otimes\left((3.124113213204101323)_{5} \times 5^{0}\right)+\zeta_{3} \otimes\left((2.114244212313224030)_{5} \times 5^{3}\right)$ \\
\hline 7 & $1 \otimes\left((6.430024313466045624)_{7} \times 7^{0}\right)+\zeta_{3} \otimes\left((5.065261416212661354)_{7} \times 7^{0}\right)$ \\
\hline 11 & $1 \otimes\left((5 . A 88931 A 0106 A 0172 A 3)_{11} \times 11^{0}\right)+\zeta_{3} \otimes\left((3 . A 4151 A 485571789978)_{11} \times 11^{0}\right)$ \\
\hline
\end{tabular}

[24] T. Dokchitser. Computing special values of motivic $L$-functions. Experiment. Math., 13(2):137-149, 2004.

[25] A. B. Goncharov. Polylogarithms and motivic Galois groups. In Motives (Seattle, WA, 1991), volume 55 of Proc. Sympos. Pure Math., pages 43-96. Amer. Math. Soc., Providence, RI, 1994.

[26] A. B. Goncharov. Geometry of configurations, polylogarithms, and motivic cohomology. Adv. Math., 114(2):197-318, 1995.

[27] A. B. Goncharov. Deninger's conjecture of $L$-functions of elliptic curves at $s=3$. J. Math. Sci., 81(3):2631-2656, 1996. Algebraic geometry, 4.

[28] R. Greenberg. On p-adic Artin L-functions. Nagoya Math. J., 89:77-87, 1983.

[29] M. Gros. Régulateurs syntomiques et valeurs de fonctions $L$ p-adiques. I. Invent. Math., 99(2):293-320, 1990. With an appendix by Masato Kurihara.

[30] M. Gros. Régulateurs syntomiques et valeurs de fonctions $L$ p-adiques. II. Invent. Math., 115(1):61-79, 1994. 
TABLE 16. Splitting field of $x^{6}-x^{5}-8 x^{4}+11 x^{3}+2 x^{2}-5 x+1$, where $C\left(\chi_{\pi} \otimes\right.$ id $)=2^{2} \cdot 3^{2} \cdot 13$.

\begin{tabular}{lc}
\hline$n=3$ & $e\left(3, M_{\pi}^{E}\right)^{-1}=-2^{-2} \cdot 3^{2} \cdot 13^{2} \cdot \zeta_{3}$ \\
\hline$p$ & $1 \otimes\left((1.101100110101111100)_{2} \times 2^{2}\right)+\zeta_{3} \otimes\left((1.001000001010110111)_{2} \times 2^{0}\right)$ \\
\hline 2 & $1 \otimes\left((2.021221201100120120)_{3} \times 3^{5}\right)+\zeta_{3} \otimes\left((2.020012210111121212)_{3} \times 3^{7}\right)$ \\
3 & $1 \otimes\left((3.000431031211204434)_{5} \times 5^{6}\right)+\zeta_{3} \otimes\left((1.012314421212444244)_{5} \times 5^{6}\right)$ \\
5 & $1 \otimes\left((3.0426445363200511)_{7} \times 7^{6}\right)+\zeta_{3} \otimes\left((1.1035504615611621)_{7} \times 7^{6}\right)$ \\
7 & $1 \otimes\left((8.9799 A 36158285)_{11} \times 11^{6}\right)+\zeta_{3} \otimes\left((9.229923 A A A 0734)_{11} \times 11^{6}\right)$ \\
11 & $L_{p}\left(3, \chi_{\pi} \otimes \omega_{p}^{-2}, \mathbb{Q}\right)$ \\
\hline$p$ & $1 \otimes\left((1.100100111001100011)_{2} \times 2^{2}\right)+\zeta_{3} \otimes\left((1.101001000011101110)_{2} \times 2^{4}\right)$ \\
\hline 2 & $1 \otimes\left((1.022110110201121122)_{3} \times 3^{2}\right)+\zeta_{3} \otimes\left((2.102110200120211001)_{3} \times 3^{0}\right)$ \\
3 & $1 \otimes\left((2.424401443414003124)_{5} \times 5^{0}\right)+\zeta_{3} \otimes\left((4.440233034214103134)_{5} \times 5^{0}\right)$ \\
5 & $1 \otimes\left((5.421026022055251515)_{7} \times 7^{0}\right)+\zeta_{3} \otimes\left((4.510213122044500212)_{7} \times 7^{0}\right)$ \\
7 & $1 \otimes\left((A .187435006878255608)_{11} \times 11^{0}\right)+\zeta_{3} \otimes\left((6.47020389393 A A 07 A 54)_{11} \times 11^{0}\right)$ \\
11 & $R_{5, p}\left(M_{\pi}^{E}\right) / D\left(M_{\pi}^{E}\right)^{1 / 2, p}$ \\
\hline \hline$n=5$ & $1 \otimes\left((1.001111100010111111)_{2} \times 2^{7}\right)+\zeta_{3} \otimes\left((1.100110001101001011)_{2} \times 2^{8}\right)$ \\
\hline$p$ & $1 \otimes\left((1.100222220001012001)_{3} \times 3^{15}\right)+\zeta_{3} \otimes\left((1.201100102012122102)_{3} \times 3^{15}\right)$ \\
\hline 2 & $1 \otimes\left((6.54022035336014)_{7} \times 7^{10}\right)+\zeta_{3} \otimes\left((1.31434160250212)_{7} \times 7^{10}\right)$ \\
3 & $1 \otimes\left((9.5 A 062420171)_{11} \times 11^{10}\right)+\zeta_{3} \otimes\left((A .873665790 A 8)_{11} \times 11^{10}\right)$ \\
5 & $L_{p}\left(5, \chi_{\pi} \otimes \omega_{p}^{-4}, \mathbb{Q}\right)$ \\
7 & $1 \otimes\left((1.101111101101011100)_{2} \times 2^{2}\right)+\zeta_{3} \otimes\left((1.001110000011000011)_{2} \times 2^{4}\right)$ \\
11 & $1 \otimes\left((2.001012111110022002)_{3} \times 3^{1}\right)+\zeta_{3} \otimes\left((2.011221210002201001)_{3} \times 3^{0}\right)$ \\
\hline$p$ & $1 \otimes\left((5.454116060262152231)_{7} \times 7^{0}\right)+\zeta_{3} \otimes\left((5.160242506230053303)_{7} \times 7^{0}\right)$ \\
\hline 2 & $\left.1(9.9950088486 A 6479846)_{11} \times 11^{0}\right)+\zeta_{3} \otimes\left((A .178492061888054088)_{11} \times 11^{0}\right)$ \\
\hline & 1
\end{tabular}

[31] U. Jannsen. Deligne homology, Hodge-D-conjecture, and motives. In Beilinson's conjectures on special values of L-functions, volume 4 of Perspect. Math., pages 305-372. Academic Press, Boston, MA, 1988.

[32] N. Katz. p-adic interpolation of real analytic Eisenstein series. Ann. of Math. (2), 104(3):459-571, 1976.

[33] N. Katz. p-adic L-functions for CM fields. Invent. Math., 49(3):199-297, 1978.

[34] N. Katz. Review of l-adic cohomology. In Motives (Seattle, WA, 1991), volume 55 of Proc. Sympos. Pure Math., pages 21-30. Amer. Math. Soc., Providence, RI, 1994.

[35] T. Kubota and H.-W. Leopoldt. Eine $p$-adische Theorie der Zetawerte. I. Einführung der p-adischen Dirichletschen L-Funktionen. J. Reine Angew. Math., 214/215:328-339, 1964.

[36] S. Lang. Algebraic number theory. Addison-Wesley Publishing Co., Inc., Reading, Mass.London-Don Mills, Ont., 1970.

[37] B. Mazur, J. Tate, and J. Teitelbaum. On p-adic analogues of the conjectures of Birch and Swinnerton-Dyer. Invent. Math., 84(1):1-48, 1986. 
[38] J. Neukirch. The Beullinson conjecture for algebraic number fields. In Beר̆linson's conjectures on special values of L-functions, volume 4 of Perspect. Math., pages 193-247. Academic Press, Boston, MA, 1988.

[39] J. Neukirch. Algebraic number theory, volume 322 of Grundlehren der Mathematischen Wissenschaften [Fundamental Principles of Mathematical Sciences]. Springer-Verlag, Berlin, 1999. Translated from the 1992 German original and with a note by Norbert Schappacher, With a foreword by G. Harder.

[40] W. Nizioł. On the image of p-adic regulators. Invent. Math., 127:375-400, 1997.

[41] B. Perrin-Riou. Fonctions $L$ p-adiques des représentations $p$-adiques. Astérisque, (229):198pp, 1995.

[42] D. Quillen. Finite generation of the groups $K_{i}$ of rings of algebraic integers. In Algebraic K-theory 1, volume 341 of Lecture Notes in Mathematics, pages 179-198. Springer Verlag, Berlin, 1973.

[43] X.-F. Roblot. Computing values of $p$-adic $L$-functions of totally real number fields. In preparation.

[44] X.-F. Roblot and D. Solomon. Verifying a $p$-adic abelian Stark conjecture at $s=1$. J. Number Theory, 107(1):168-206, 2004.

[45] P. Schneider. Introduction to the Beilinson Conjectures. In Beilinson's Conjectures on Special Values of L-Functions, pages 1-35. Academic Press, Boston, MA, 1988.

[46] A. J. Scholl. An introduction to Kato's Euler systems. In Galois representations in arithmetic algebraic geometry (Durham, 1996), pages 379-460. Cambridge Univ. Press, Cambridge, 1998.

[47] J.-P. Serre. Formes modulaires et fonctions zêta $p$-adiques. In Modular functions of one variable, III (Proc. Internat. Summer School, Univ. Antwerp, 1972), pages 191-268. Lecture Notes in Math., Vol. 350. Springer, Berlin, 1973.

[48] T. Shintani. On evaluation of zeta functions of totally real algebraic number fields at nonpositive integers. J. Fac. Sci. Univ. Tokyo Sect. IA Math., 23(2):393-417, 1976.

[49] C. Soulé. On higher p-adic regulators. In Algebraic K-theory, Evanston 1980 (Proc. Conf., Northwestern Univ., Evanston, Ill., 1980), volume 854 of Lecture Notes in Math., pages 372-401. Springer Verlag, Berlin, 1981.

[50] A. A. Suslin. $K_{3}$ of a field, and the Bloch group. Trudy Mat. Inst. Steklov., 183:180-199, 229, 1990. Translated in Proc. Steklov Inst. Math. 1991, no. 4, 217-239, Galois theory, rings, algebraic groups and their applications (Russian).

[51] The Magma group, Sydney. Magma. Available from http://magma.maths.usyd.edu.au/.

[52] The PARI Group, Bordeaux. PARI/GP, version 2.2.11, 2005. Available from http:// pari.math.u-bordeaux.fr/.

[53] A. Wiles. The Iwasawa conjecture for totally real fields. Ann. of Math. (2), 131(3):493-540, 1990.

[54] D. Zagier. Polylogarithms, Dedekind Zeta Functions and the Algebraic $K$-theory of Fields. In Arithmetic algebraic geometry (Texel, 1989), pages 391-430. Birkhäuser Boston, Boston, MA, 1991. 
Amnon Besser

Department of Mathematics

Ben-Gurion University of the Negev

P.O.B. 653

Be'er-Sheva 84105

Israel

Paul Buckingham

Department of Pure Mathematics

University of Sheffield

Hicks Building

Hounsfield Road

Sheffield S3 7RH

United Kingdom

Rob de Jeu

Faculteit der Exacte Wetenschappen

Afdeling Wiskunde

Vrije Universiteit

De Boelelaan 1081a

1081 HV Amsterdam

The Netherlands

E-mail: jeu@few.vu.nl

Xavier-François Roblot

Institut Camille Jordan

Université de Lyon, Université Lyon 1, CNRS - UMR 5208

43 blvd du 11 Novembre 1918, 69622 Villeurbanne Cedex

France 